\title{
ESTUDO DOS EFEITOS CONSTITUTIVOS SOBRE A ESTABILIDADE ELÁSTICA DE PERFIS METÁLICOS
}

Dissertação apresentada à Escola Politécnica da Universidade de São Paulo para obtenção do título de Mestre em Engenharia. 


\section{ESTUDO DOS EFEITOS CONSTITUTIVOS SOBRE A ESTABILIDADE ELÁSTICA DE PERFIS METÁLICOS}

Dissertação apresentada à Escola Politécnica da Universidade de São Paulo para obtenção do título de Mestre em Engenharia.

Área de concentração: Engenharia de Estruturas Orientador: Prof. Dr. Eduardo M. B. Campello 


\section{AGRADECIMENTOS}

Agradeço primeiramente a Deus pela saúde e por ter me dado uma família maravilhosa que me permitiu ser o que sou hoje. A religião espírita, por me fazer sempre entender um pouco mais sobre o sentido da nossa vida aqui neste plano material.

Aos meus amados pais Valmir e Neide, por sempre estarem ao meu lado em todos os momentos da minha vida, me dando apoio, incentivo, consolo, conselhos, carinho e muito amor. Sem eles eu jamais teria chegado até aqui. Em especial ao meu pai, que é o meu maior exemplo de profissional e meu ídolo. A minha mãe por ser a melhor amizade que tenho em vida. Eles fizeram do meu sonho os sonhos deles.

As minhas amadas irmãs Larissa e Lorena Lago, por todo amor e amizade dada durante esta jornada e durante todas nossas vidas como irmãos. E as minhas primas irmãs Mônica e Regina por todo o carinho e amor, e pela compreensão da minha ausência nos momentos importantes da nossa família neste período, principalmente nos acontecimentos nas vidas dos meus amados sobrinhos Rafael, Arthur, Isadora e Maria Eduarda.

A minha amada tia Martha, minha segunda mãe, por todo amor, apoio, ajuda e motivação dada a mim durante toda minha vida.

Ao Prof. Dr Eduardo Campello pela tamanha dedicação dada na sua orientação neste trabalho. Pelo exemplo como professor e profissional acadêmico demonstrando sempre paixão pelo que faz, além do grande conhecimento técnico. E acima de tudo pela amizade e paciência durante todas as etapas da realização deste trabalho. Pelas conversas fora da vida acadêmica sempre nos motivando a vencer e nunca desistir. Aqui não somente tive um orientador, mas fiz um grande amigo. Alguém que me inspirou a seguir a carreira acadêmica.

Aos meus amigos do JAC (JOHN ARGYRIS CENTER), Marcelo, Cinthia, Henrique, Márcia, Paulo, Eduardo, Alexandre, Fernando e Jorge por toda amizade durante o período do mestrado. Em especial aos amigos Jorge e Fernando por todo apoio técnico durante esta jornada, acima de tudo pela grande amizade. E ao amigo Alexandre por todas as conversas sobre vida e pela amizade aqui construída. Neste grupo não encontrei somente amigos, mas sim uma família que pretendo sempre está perto.

Aos meus amigos do LMC-EPUSP (Laboratório de Mecânica Computacional), a querida Gabriela, ao casal de amigos Nicolau e Michele a quem desejo toda felicidade do mundo, e em especial ao meu grande amigo irmão Dr Igor Pierin pela grande amizade, ajuda e apoio.

Aos amigos do LEM, Luís e Ladislao por toda a amizade. As amigas Dras Luciana e Carol pela grande amizade que construímos ao longo desses anos, com tantas conversas em períodos de felicidade e tristeza, sempre prevalecendo a nossa amizade.

Aos meus mestres de karatê, sensei Yoshizo Machida e sensei Yasuyuki Sasaki, por todos os ensinamentos dentro da arte marcial que sempre me ajudaram na vida. Sempre mostrando o caminho do Budô e o verdadeiro sentido da faixa preta - o início da jornada sem fim de disciplina assim como o mestrado. E a toda filosofia que aprendi também com a prática do taekwondo ao longo de tantos anos, me ajudando a trilhar sempre o caminho em busca da vitória. 
DOJO KUN

Esforçar-se para formação do caráter Fidelidade para com o verdadeiro caminho da razão

Criar o intuito de esforço Respeito acima de tudo Conter o espírito de agressão

"Quem conhece os outros é inteligente Quem conhece a si mesmo é sábio

Quem vence os outros é forte

Quem vence a si mesmo é poderoso Quem se faz valer tem força de vontade

Quem é auto suficiente é rico

Quem não perde o seu lugar é estável Quem mesmo na morte não perece, esse vive.” 


\section{RESUMO}

Este trabalho utiliza uma teoria não linear tridimensional de barras de [1], que considera o empenamento da seção transversal como uma das variáveis do problema, para a análise de perfis de aço por meio do Método dos Elementos Finitos.

A equação constitutiva apresentada em [2] é aqui expressa de forma completa, com os termos de ordem cúbica e superiores antes desprezados. A influência da inclusão desses termos sobre a estabilidade da barra pode assim ser estudada com maior profundidade.

O modelo constitutivo obtido foi implementado em um programa computacional de elementos finitos, e diversos exemplos numéricos são apresentados para validar a teoria aqui desenvolvida. Foi possível obter a instabilidade por flexo-torção com a equação constitutiva “completa” aqui desenvolvida, onde antes não era possível em [2]. Melhorando o modelo constitutivo. 


\begin{abstract}
This work presents a nonlinear tridimensional beam theory of [1], which encompasses crosssectional warping as one of the model variables, and its application to the analysis of steel profiles with the Finite Element Method.

The constitutive equation presented in [2] is fully developed, including the third and higher order terms neglected therein. The influence of the inclusion of such terms on the beam stability could then be studied more deeply.

The obtained constitutive model was implemented in a computational Finite Element program and several numerical examples are presented to validate the theory here developed. The complete constitutive equation made possible to determine instability in composite flexuraltorsional states, a clear contribution to the model developed in [2].
\end{abstract}




\section{ÍNDICE}

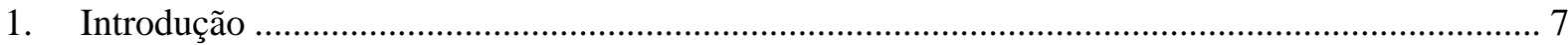

2. Formulação cinematicamente exata de barras com implementação em elementos finitos .............12

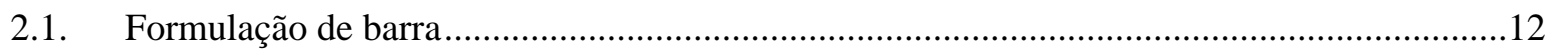

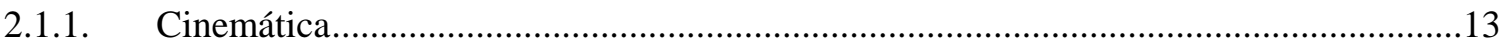

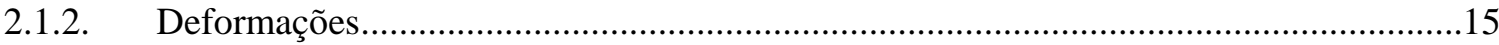

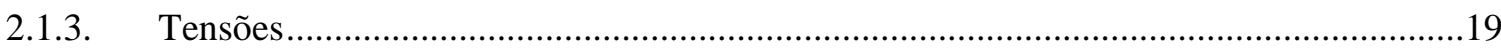

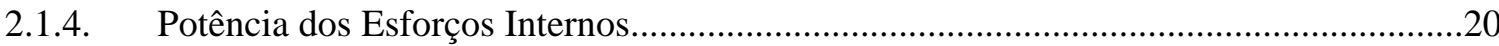

2.1.5. Potência dos Esforços Externos ………………...............................................................22

2.1.6. Equações de Equilíbrio e Condições de Contorno ............................................................23

2.1.7. Linearização dos trabalhos virtuais: operador Tangente ..................................................25

2.2. Implementação em método dos elementos finitos..................................................................27

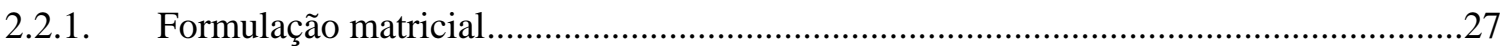

2.2.2. Formulação das matrizes globais e solução do problema .................................................31

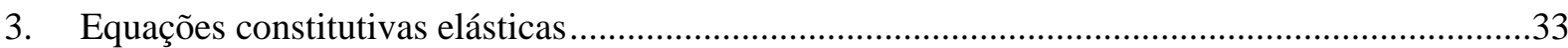

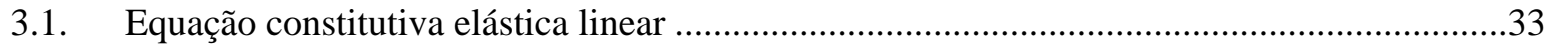

3.2. Equação constitutiva hiperelástica “completa” de Kirchhoff-St-Venant ................................36

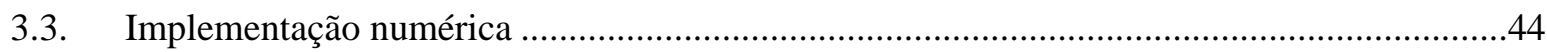

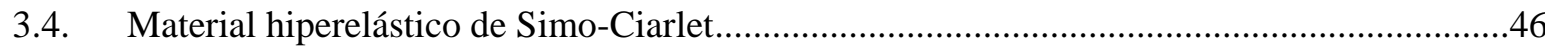

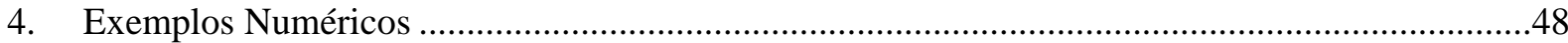

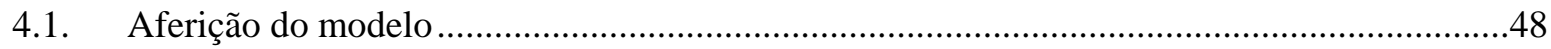

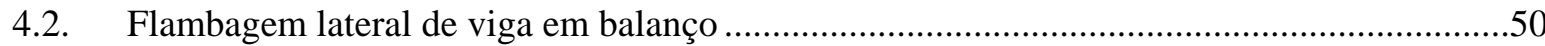

4.3. Flambagem de pilares por compressão com seção I e retangular ............................................52

4.4. Flambagem de pilares por compressão com seção cruciforme …..........................................54

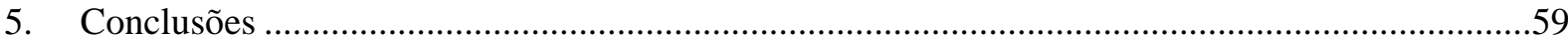

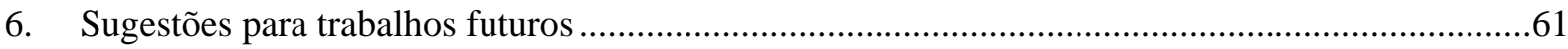

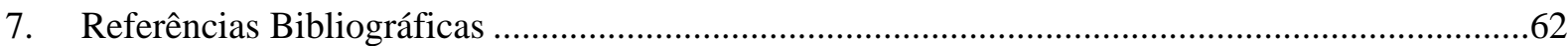




\section{Introdução}

Em [3], explica-se que o primeiro material siderúrgico empregado na construção foi o ferro fundido, entre 1780 e 1820, sendo utilizado na construção de pontes em arco e treliças nos elementos sujeitos à compressão. Os elementos que resistiam aos esforços de tração eram feitos de ferro forjado que, além da excelente resistência à tração, apresentava boa resistência à corrosão.

As estruturas metálicas têm registro de sua utilização em escala industrial a partir de 1750 . No Brasil, o ferro fundido começou a ser produzido em 1812, e a primeira obra em ferro fundido, moldada no Estaleiro Mauá, em Niterói, no estado do Rio de Janeiro, foi a Ponte de Paraíba do Sul, no Estado do Rio, com cinco vãos de 30 metros, estruturados em arcos atirantados, com o arco em ferro fundido e o tirante em ferro laminado, construída em 1857, estando em uso até hoje, sendo que o grande avanço na fabricação de perfis em larga escala ocorreu com a implementação das grandes siderúrgicas. Somente após a 2a. Guerra Mundial com a construção da Usina de Volta Redonda no Rio de Janeiro, a Indústria Siderúrgica implantouse de fato no Brasil.

O emprego de estruturas de aço no Brasil, todavia ainda pouco intenso, vem aumentando, em particular nos edifícios comercias e nos de uso industrial, nas residências de arquitetura mais moderna e nas estruturas de apoio de equipamentos.

Sendo os perfis metálicos elementos fabricados pela indústria com dimensões padronizadas, seu dimensionamento baseia-se na escolha do melhor elemento existente, não na elaboração da geometria ideal para cada caso. Dessa forma, o desafio do profissional de quem trabalha com projetos de estrutura de aço é a escolha do perfil que mais se adequa às suas necessidades.

As estruturas metálicas possuem diversas vantagens, tais como:

- qualidade uniforme, aliada à alta resistência mecânica, possibilitando a execução de estruturas leves para vencer grandes vãos;

- facilidade, rapidez e economia na fabricação, no manuseio e montagem, com a consequente redução nos custos financeiros e nas despesas gerais de canteiro e de administração;

- execução das estruturas com precisão milimétrica permitindo um alto controle de qualidade do produto acabado; 
- economia nas fundações, pela redução do peso próprio da estrutura;

- em caso de necessidade, podem-se desmontar as estruturas para posterior montagem em outro local;

- não exigem mão de obra especializada para a montagem, desde que as ligações de obra sejam através de parafusos;

- além disso, segundo [4], os elementos de aço oferecem uma grande margem de segurança no trabalho, o que se deve ao fato de o material ser único e homogêneo, com limite de escoamento, ruptura e módulo de elasticidade bem definidos.

Devido às características geométricas desses perfis, a estabilidade lateral e o empenamento das seções transversais tornam-se fatores importantes na etapa de análise estrutural. Muitas das metodologias usuais de cálculo e dimensionamento, no entanto, não contemplam adequadamente esses aspectos, fazendo com que às vezes resultem em estruturas pouco econômicas ou até mesmo com pouca ou nenhuma segurança.

A análise não linear de sistemas estruturais tem sido um assunto bastante pesquisado nas últimas duas décadas, tendo conquistado importância prática e aplicações cada vez maiores nos ramos da Engenharia.

Segundo [5], o cálculo estrutural teve seu maior desenvolvimento a partir dos meados do século XX, com o advento dos computadores.

Os métodos de análise estrutural, segundo [6], podem ser analíticos e numéricos. Os métodos analíticos, apesar de apresentarem soluções fechadas, de grande utilidade, são muito limitados em aplicações mais complexas [7]. Dos métodos numéricos, o que apresentou maior evolução foi o método matricial, havendo culminado no método dos elementos finitos, cujo desenvolvimento se deve a [8], principalmente, a [9] e [10] no período de 1955 a 1960. Desta época até os tempos atuais, as teorias envolvendo sólidos tridimensionais, estados planos de tensão e deformação, cascas, placas e barras tiveram grandes desenvolvimentos e vêm resolvendo problemas estruturais, até então impossíveis de serem solucionados analiticamente.

Nesse contexto, as teorias estruturais para barras também tiveram um desenvolvimento bem acentuado. Uma das teorias, cujas aplicações são de fundamental importância, é a teoria geometricamente ou cinematicamente exata de barras tridimensionais, pois esta apresenta uma 
efetiva interação entre a estabilidade da estrutura e os critérios de resistência dos materiais de forma sistemática, sem necessidade de impor restrições de qualquer ordem. Muitos conceitos ligados à teoria linear são fundamentais para esclarecer a teoria exata, a qual não deve ser entendida como simples generalização da teoria linear.

Até a década de 70 e meados dos anos 80, a teoria exata para barras tridimensionais, que não é apenas uma generalização da teoria no plano, era mal compreendida pelos estudiosos da época, como em [11] e [12]. A dificuldade da teoria se deve à manipulação das rotações, que no espaço tridimensional não é uma operação comutativa mesmo para pequenas rotações [7]. Em [13] e [14], foram apresentadas as primeiras teorias não lineares de barra geometricamente exatas, obtidas a partir de uma generalização da teoria apresentada por Kirchhoff em 1859. Entretanto, não demonstravam consistência clara com os princípios da Mecânica dos Sólidos Deformáveis. Sua real implantação como teorias consistentes neste sentido, envolvendo grandes rotações e com dedução de equações não lineares sem nenhuma aproximação cinemática, conhecidas como teorias geometricamente exatas, foi feita em [15], [16], [17], [18] e [19] para barras tridimensionais.

Em [15] foi apresentada uma formulação não linear para barras no espaço, sem aproximações geométricas, contendo deduções consistentes das equações do movimento (ou equilíbrio dinâmico). Em [16], por sua vez, foram deduzidas as equações não lineares de equilíbrio estático para barras tridimensionais sujeitas às hipóteses cinemáticas do modelo de Timoshenko, detalhando-se ainda a obtenção do operador tangente [20].

Em 1998 [21] propôs uma formulação para barras curvas tridimensionais de seção transversal qualquer, comportando situações de grandes deslocamentos e grandes rotações. Foram feitas algumas aproximações para considerar o empenamento da seção na cinemática do problema, como em [22] e [7]. Entretanto, a formulação somente é válida para os casos de pequenas deformações.

Em [22] foi feito uma análise comparativa entre a teoria de Vlasov [23] e a teoria não linear geometricamente exata formulada por [19], onde se verificou certos erros fazendo com que o autor propulse-se uma alteração na hipótese constitutiva desta última. A expressão linearizada da segunda variação da energia potencial foi obtida após esta modificação e comparada com a expressão obtida a partir da teoria de Vlasov. São apontadas as principais diferenças entre os termos geométricos e os termos constitutivos não lineares de ambas. 
No trabalho de [1], como uma generalização de [19], apresentou-se uma teoria estrutural geometricamente exata para barras retas no espaço que considera o empenamento não uniforme da seção transversal como umas das variáveis do problema. Foram consideradas equações constitutivas elásticas lineares e quadráticas, sendo que neste último caso os termos quadráticos referentes às deformações de empenamento foram desprezados (todos os outros foram retidos). Foi proposta uma forma alternativa de parametrizar as rotações no espaço tridimensional, mostrando-se que é computacionalmente mais vantajosa do que a maneira clássica de Euler-Rodrigues.

Em [2], foi utilizada a mesma teoria de barras de [1], porém considerando todos os termos de segunda ordem na parte constitutiva. Alguns modelos hiperelásticos foram discutidos, mostrando que os termos de segunda ordem na equação constitutiva podem ter efeitos consideráveis sobre a estabilidade da barra, confirmando o que havia sido sugerido em [24] para barras sem empenamento.

Dentro deste contexto, este trabalho de mestrado faz a generalização da equação constitutiva de [2] para incorporar todos os termos de ordem superior (cúbicos em diante) na equação constitutiva. Busca-se estudar em maior profundidade a influência desses termos sobre a estabilidade da barra.

O modelo constitutivo assim obtido chamado de "equação constitutiva completa" foi implementado no programa computacional PEFSYS, é um programa de elementos finitos, implementado em linguagem Fortran 90, para análise não linear de sólidos e estruturas, desenvolvido na Escola Politécnica da Universidade de São Paulo pelo grupo de pesquisadores do qual o autor faz parte, para análise não linear de sólidos e estruturas.

Simulações numéricas foram empreendidas, especialmente problemas de flambagem de vigas metálicas e pilares com diferentes tipos de condições de contorno, para avaliar o efeito dos termos constitutivos superiores sobre as cargas críticas e sobre o comportamento pós-crítico.

Este trabalho é uma extensão de dois trabalhos anteriores, [2] e [25], no sentido de que ele apresenta a equação constitutiva elástica “completa” para a análise de barras com graus de liberdade empenamento. Derivou-se a equação constitutiva a partir do modelo tridimensional hiperelástico de Kirchhoff-Saint-Venant, relacionando o segundo tensor de Piola-Kirchhoff com o tensor das deformações de Green-Lagrange. Em contraste com [2] e [25], no entanto, todos os termos de ordem superior (cúbicos e acima) nas deformações são retidos no nível 
constitutivo. As tensões assim obtidas são transformadas em tensões nominais (isto é, em tensões do primeiro tensor de Piola Kirchhoff) e depois integradas na seção transversal, fornecendo os esforços resultantes da seção transversal. Os correspondentes módulos elásticos de rigidez tangente (ou seja, as derivadas dos esforços resultantes com relação às deformações da seção transversal) são obtidos de maneira exata.

Uma vez que são retidos todos os termos nas deformações, a integração das tensões na seção transversal gera várias propriedades geométricas de ordem superior da seção transversal. Lida-se com essas “pesadas” quantidades por meio de integração numérica. No entanto, como conseqüência, a expressão explícita da função empenamento passa a ser necessária, e isso coloca uma desvantagem dentro do presente modelo. Aqui, propõe-se a adoção de uma função empenamento que é uma combinação da área setorial de Vlasov [23] com a função bicúbica de [26], contemplando tanto o empenamento primário quanto o chamado empenamento secundário ou empenamento na espessura.

Lembrando que neste trabalho se preocupou somente em melhorar a equação constitutiva elástica, não tratando de tensões residuais, elastoplasticidade ou efeitos locais 3D.

O texto está organizado da seguinte forma: no Capítulo 2, apresenta-se uma formulação cinematicamente exata de barras com implementação em elementos finitos. No Capítulo 3 são apresentados as equações constitutivas usadas neste trabalho e o desenvolvimento da equação contistitutiva “completa”. Os exemplos numéricos para validação da pesquisa são apresentados no Capítulo 4 e finalmente, as conclusões do trabalho são comentadas no Capítulo 5.

Com relação à notação utilizada, ao longo de todo o trabalho letras minúsculas latinas ou gregas em itálico $(a, b, \ldots, \alpha, \beta, \ldots)$ representam grandezas escalares, ao passo que as correspondentes em negrito $(\boldsymbol{a}, \boldsymbol{b}, \ldots, \boldsymbol{\alpha}, \boldsymbol{\beta}, \ldots)$ denotam vetores. Letras maiúsculas latinas ou gregas em negrito-itálico $(\boldsymbol{A}, \boldsymbol{B} \ldots)$ expressam tensores de segunda ordem, todos no espaço Euclidiano tridimensional. Vetores e matrizes construídos com componentes tensoriais (por exemplo para fins computacionais) são expressos por letras latinas em negrito não-itálico $(\mathbf{A}, \mathbf{B} \ldots \mathbf{a}, \mathbf{b}, \ldots)$. Adota-se ainda a convenção de somatório sobre índices repetidos, estando subentendido que esses tomam valores entre $\{1,2\}$ para letras gregas ou entre $\{1,2,3\}$ para letras latinas. 


\section{Formulação cinematicamente exata de barras com implementação em elementos finitos}

\subsection{Formulação de barra}

Em condições de linearidade geométrica, ou seja, situações de pequenos deslocamentos e pequenas rotações, várias teorias de barras foram introduzidas ao longo dos anos. Dentre elas destacam-se algumas da primeira metade do século XX, como a Teoria de Timoshenko [27] e a Teoria de Vlasov [23]. A última teve relevância por incluir o empenamento da seção transversal na cinemática da barra, contemplando o problema da torção não uniforme.

No trabalho de [15], apresentou-se uma formulação não linear para barras no espaço sem aproximações geométricas, contendo deduções consistentes das equações do movimento (ou equilíbrio dinâmico). Em [16], foram deduzidas as equações exatas de equilíbrio estático para barras tridimensionais sujeitas às hipóteses cinemáticas do modelo de Timoshenko, detalhando-se ainda a obtenção do operador tangente. Em [19], o operador tangente para esses tipos de teoria foi deduzido pela primeira vez de forma correta, e em [17] foi feito a sua extensão para incorporar o empenamento.

Este capítulo apresenta a teoria de barras cinematicamente (ou geometricamente) exata de [7] e [28] (e que teve sua implementação numérica efetuada em [1]), que incorpora o empenamento das seções transversais na descrição cinemática da barra. A teoria utiliza formulação Lagrangiana total e emprega como grandezas fundamentais o primeiro tensor das tensões de Piola-Kirchhoff e o gradiente das deformações. As variáveis utilizadas para o desenvolvimento das equações são grandezas relativas à configuração de referência, formadas por componentes dos vetores dos deslocamentos e rotações.

Por se uma formulação geometricamente exata é adequada tanto à análise de tensões e deslocamentos quanto à análise de estabilidade.

É considerado o efeito da distorção devido à força cortante e também o empenamento não uniforme das seções transversais devido à flexotorção. 


\subsubsection{Cinemática}

Nesta teoria, a hipótese cinemática fundamental é a de que as seções transversais inicialmente ortogonais ao eixo da barra na configuração de referência permanecem indeformáveis na projeção em seu plano durante o movimento, mas não necessariamente ortogonais ao eixo deformado, devido aos efeitos da força cortante. Além disso, podem deixar de ser planas, devido à consideração do empenamento. Os pontos situados na seção transversal da barra sofrem primeiramente um deslocamento e uma rotação, e então é superposto um deslocamento na direção ortogonal à seção deformada, correspondendo ao empenamento.

A Figura 2.1 mostra a representação do movimento da barra no espaço. Na configuração de referência, ou indeformada, define-se uma base local ortonormal de vetores $\left\{\boldsymbol{e}_{1}^{r}, \boldsymbol{e}_{2}^{r}, \boldsymbol{e}_{3}^{r}\right\}$, com $\boldsymbol{e}_{3}^{r}$ coincidente com o eixo da barra. A barra é admitida reta na sua posição indeformada e tem comprimento inicial $l$. O índice " $r$ " sobre uma grandeza indica que ela se encontra na configuração de referência.

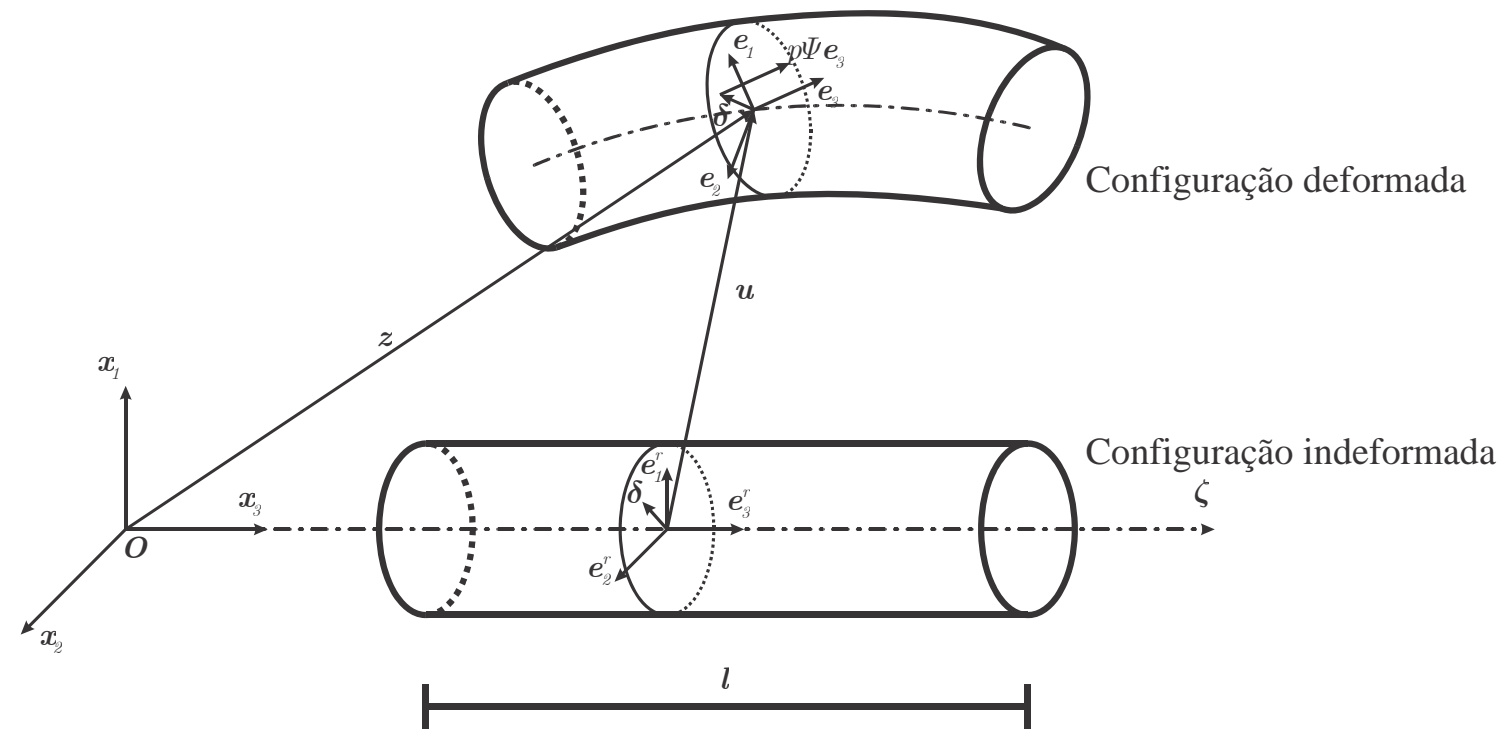

Figura 2.1- Descrição da barra e grandezas cinemáticas fundamentais (figura adaptada de [29]).

Os pontos da barra na configuração de referência podem ser descritos vetorialmente por:

$$
\boldsymbol{\xi}=\boldsymbol{\zeta}+\boldsymbol{a}^{r}
$$


onde:

$$
\zeta=\zeta e_{3}^{r}, \zeta \in[0, l]
$$

descreve a posição dos pontos do eixo e

$$
\boldsymbol{a}^{r}=x_{\alpha} e_{3}^{r}
$$

descreve a posição dos demais pontos da seção transversal em relação ao eixo.

Depois da deformação, define-se outra base $\left\{\boldsymbol{e}_{1}, \boldsymbol{e}_{2}, \boldsymbol{e}_{3}\right\}$ ortonormal de vetores, chamada de base local móvel, com as seções transversais contidas no plano $\left\{\boldsymbol{e}_{1}, \boldsymbol{e}_{2}\right\}$. Os pontos da barra passam a ser descritos por uma nova função vetorial $\boldsymbol{x}=\hat{\boldsymbol{x}}(\boldsymbol{\xi})$, dada por

$$
\boldsymbol{x}=\boldsymbol{z}+\boldsymbol{a}+\psi p \boldsymbol{e}_{3} .
$$

Da observação da Figura 2.1, pode-se notar que $\boldsymbol{z}=\hat{\boldsymbol{z}}(\boldsymbol{\zeta})$, que descreve a nova posição dos pontos do eixo da barra, e $\boldsymbol{a}=\hat{\boldsymbol{a}}(\boldsymbol{\xi})$ descreve a posição dos demais pontos da seção em relação ao eixo deformado. Além disso $\psi=\hat{\psi}\left(x_{\alpha}\right)$ é a função empenamento relativa ao centro de cisalhamento, e $p=\hat{p}(\boldsymbol{\zeta})$ é o parâmetro escalar associado a $\psi$ que dá sua intensidade.

O vetor $\boldsymbol{a}$ é dado por

$$
\boldsymbol{a}=\boldsymbol{Q \boldsymbol { a } ^ { r }}=x_{\alpha} \boldsymbol{e}_{\alpha}
$$

sendo $\boldsymbol{Q}=\hat{\boldsymbol{Q}}(\boldsymbol{\zeta})$ o tensor ortogonal das rotações das seções transversais. A expressão (2.5) mostra que $\boldsymbol{a}$ é nada mais que $\boldsymbol{a}^{r}$ rotacionado de $\boldsymbol{Q}$.

O tensor $\boldsymbol{Q}$ pode ser obtido através da fórmula de Euler-Rodrigues [30], [31], detalhada pela expressão abaixo:

$$
\boldsymbol{Q}=\boldsymbol{I}+\frac{\operatorname{sen} \theta}{\theta} \boldsymbol{\Theta}+\frac{1}{2} \frac{\operatorname{sen}^{2}(\theta / 2)}{(\theta / 2)^{2}} \boldsymbol{\Theta}^{2},
$$


onde $\Theta$ é um tensor antissimétrico cujo vetor axial é $\boldsymbol{\theta}$, sendo este último o chamado vetor rotação que caracteriza a rotação da seção tranversal, cuja magnitude é $\theta=\|\boldsymbol{\theta}\|$.

Pode-se determinar o vetor dos deslocamentos $\boldsymbol{u}$ de um ponto do eixo da barra usando a expressão:

$$
u=z-\zeta
$$

Já o vetor dos deslocamentos de um ponto genérico da barra pode ser expresso por

$$
\boldsymbol{\delta}=\boldsymbol{x}-\boldsymbol{\xi}
$$

\subsubsection{Deformações}

O gradiente da transformação, para a deformação da barra, pode ser representado por

$$
\boldsymbol{F}=\frac{\partial \boldsymbol{x}}{\partial \boldsymbol{\xi}}=\frac{\partial \boldsymbol{x}}{\partial x_{\alpha}} \otimes \boldsymbol{e}_{\alpha}^{r}+\boldsymbol{x}^{\prime} \otimes \boldsymbol{e}_{3}^{r},
$$

em que a notação $\partial(\bullet) / \partial \zeta$ foi introduzida.

Fazendo separadamente as duas diferenciações acima, chega-se a:

$$
\begin{aligned}
\frac{\partial \boldsymbol{x}}{\partial x_{\alpha}} & =\frac{\partial\left(\boldsymbol{z}+\boldsymbol{a}+\psi p \boldsymbol{e}_{3}\right)}{\partial x_{\alpha}}=\frac{\partial\left(\boldsymbol{Q} \boldsymbol{a}^{r}+\psi p \boldsymbol{e}_{3}\right)}{\partial x_{\alpha}} \\
& =\boldsymbol{Q} \boldsymbol{e}_{\alpha}^{r}+\psi_{, \alpha} p \boldsymbol{e}_{3}=\boldsymbol{e}_{\alpha}+\psi_{, \alpha} p \boldsymbol{e}_{3}
\end{aligned}
$$

e

$$
\begin{aligned}
\boldsymbol{x}^{\prime} & =\boldsymbol{z}^{\prime}+\boldsymbol{a}^{\prime}+\left(\psi p \boldsymbol{e}_{3}\right)^{\prime} \\
& =\boldsymbol{z}^{\prime}+\boldsymbol{Q}^{\prime} \boldsymbol{a}^{r}+\psi p^{\prime} \boldsymbol{e}_{3}+\psi p \boldsymbol{Q}^{\prime} \boldsymbol{e}_{3} \\
& =\boldsymbol{z}^{\prime}+\boldsymbol{Q}^{\prime} \boldsymbol{Q}^{T} \boldsymbol{a}+\psi p^{\prime} \boldsymbol{e}_{3}+\psi p \boldsymbol{Q}^{\prime} \boldsymbol{Q}^{T} \boldsymbol{e}_{3} \\
& =\boldsymbol{z}^{\prime}+\boldsymbol{K} \boldsymbol{a}+\psi p^{\prime} \boldsymbol{e}_{3}+\psi p \boldsymbol{K} \boldsymbol{e}_{3},
\end{aligned}
$$

sendo que $\boldsymbol{K}=\boldsymbol{Q}^{\prime} \boldsymbol{Q}^{T}$ é um tensor antissimétrico cujo vetor axial é designado por $\boldsymbol{k}$.

Através de (2.7), pode-se chegar a:

$$
z^{\prime}=u^{\prime}+\zeta^{\prime}=u^{\prime}+e_{3}^{r}
$$


Definindo-se convenientemente um vetor $\boldsymbol{\eta}$ de deformações como sendo

$$
\eta=z^{\prime}-e_{3}=u^{\prime}+e_{3}^{r}-e_{3}
$$

e com ajuda dos vetores

$$
\begin{aligned}
\boldsymbol{\eta}^{r} & =\boldsymbol{Q}^{T} \boldsymbol{\eta} \\
\boldsymbol{\kappa}^{r} & =\boldsymbol{Q}^{T} \boldsymbol{\kappa},
\end{aligned}
$$

Após algumas manipulações chega-se então à seguinte forma do gradiente das deformações $\boldsymbol{F}:$

$$
\begin{aligned}
\boldsymbol{F} & =\boldsymbol{Q}\left\{\boldsymbol{I}+\psi_{, \alpha} p \boldsymbol{e}_{3}^{r} \otimes \boldsymbol{e}_{\alpha}^{r}+\left[\boldsymbol{\eta}^{r}+\boldsymbol{\kappa}^{r} \times\left(\boldsymbol{a}^{r}+\psi p \boldsymbol{e}_{3}^{r}\right)+\psi p^{\prime} \boldsymbol{e}_{3}^{r}\right] \otimes \boldsymbol{e}_{3}^{r}\right\} \\
& =\boldsymbol{Q}\left\{\boldsymbol{I}+\psi_{, \alpha} p \boldsymbol{e}_{3}^{r} \otimes \boldsymbol{e}_{\alpha}^{r}+\boldsymbol{\gamma}^{r} \otimes \boldsymbol{e}_{3}^{r}\right\}
\end{aligned}
$$

onde

$$
\boldsymbol{\gamma}^{r}=\boldsymbol{\eta}^{r}+\boldsymbol{\kappa}^{r} \times\left(\boldsymbol{a}^{r}+\psi p \boldsymbol{e}_{3}^{r}\right)+\psi p^{\prime} \boldsymbol{e}_{3}^{r}
$$

é o vetor das deformações em um ponto qualquer da seção seção transversal.

A velocidade de um ponto genérico da barra é dada pela diferenciação de seu deslocamento no tempo, ou seja:

$$
\begin{aligned}
\dot{\boldsymbol{\delta}} & =(\boldsymbol{x}-\boldsymbol{\xi})^{\bullet}=\dot{\boldsymbol{x}} \\
& =\dot{\boldsymbol{z}}+\dot{\boldsymbol{a}}+\left(\psi p \boldsymbol{e}_{3}\right)^{\bullet} \\
& =\dot{\boldsymbol{u}}+\dot{\boldsymbol{Q}} \boldsymbol{a}^{r}+\psi \dot{p} \boldsymbol{e}_{3}+\psi p \dot{\boldsymbol{Q}} \boldsymbol{e}_{3}^{r} \\
& =\dot{\boldsymbol{u}}+\dot{\boldsymbol{Q}} \boldsymbol{Q}^{T} \boldsymbol{a}+\psi \dot{p} \boldsymbol{e}_{3}+\psi p \dot{\boldsymbol{Q}} \boldsymbol{Q}^{T} \boldsymbol{e}_{3} \\
& =\dot{\boldsymbol{u}}+\boldsymbol{\Omega}\left(\boldsymbol{a}+\psi p \boldsymbol{e}_{3}\right)+\psi \dot{p} \boldsymbol{e}_{3}=\dot{\boldsymbol{u}}+\boldsymbol{\omega} \times\left(\boldsymbol{a}+\psi p \boldsymbol{e}_{3}\right)+\psi \dot{p} \boldsymbol{e}_{3},
\end{aligned}
$$

onde $\boldsymbol{\Omega}=\dot{\boldsymbol{Q}} \boldsymbol{Q}^{T}$ é o tensor antissimétrico das velocidades angulares cujo vetor axial é designado por $\boldsymbol{\omega}$. Fazendo diferenciação de (2.6) no tempo e multiplicando por $\boldsymbol{Q}^{T}$, após algumas manipulações, obtém-se a expressão abaixo para o vetor das velocidades angulares:

$$
\omega=\Gamma \dot{\boldsymbol{\theta}}
$$


com

$$
\boldsymbol{\Gamma}=\boldsymbol{I}+\frac{1}{2} \frac{\operatorname{sen}^{2}(\theta / 2)}{(\theta / 2)^{2}} \boldsymbol{\Theta}+\left(1-\frac{\operatorname{sen} \theta}{\theta}\right) \frac{1}{\theta^{2}} \boldsymbol{\Theta}^{2}
$$

Dessa forma, pode-se escrever a velocidade de um ponto da barra como

$$
\dot{\boldsymbol{\delta}}=\dot{\boldsymbol{u}}+(\boldsymbol{\Gamma} \dot{\boldsymbol{\theta}}) \times\left(\boldsymbol{a}+\psi p \boldsymbol{e}_{3}\right)+\psi \dot{p} \boldsymbol{e}_{3}
$$

Analogamente, tem-se que

$$
\kappa=\Gamma \boldsymbol{\theta}^{\prime}
$$

de maneira que

$$
\boldsymbol{\kappa}^{r}=\boldsymbol{Q}^{T} \boldsymbol{\kappa}=\boldsymbol{Q}^{T} \boldsymbol{\Gamma} \boldsymbol{\theta}^{\prime}=\boldsymbol{\Gamma}^{T} \boldsymbol{\theta}^{\prime}
$$

já que $\dot{\boldsymbol{Q}}^{\prime}=(\dot{\boldsymbol{K}}+\boldsymbol{K} \boldsymbol{\Omega}) \boldsymbol{Q}=\left(\boldsymbol{\Omega}^{\prime}+\boldsymbol{\Omega} \boldsymbol{K}\right) \boldsymbol{Q}$, pois a ordem da diferenciação não altera o resultado. Portanto, tem-se que $\boldsymbol{\Omega}^{\prime}=\dot{\boldsymbol{K}}+\boldsymbol{K} \boldsymbol{\Omega}-\boldsymbol{\Omega} \boldsymbol{K}$, o que conduz

$$
\omega^{\prime}=\dot{\boldsymbol{\kappa}}-\boldsymbol{\omega} \times \boldsymbol{\kappa} .
$$

Através da diferenciação no tempo de (2.15), ou pela diferenciação no espaço de (2.17), e usando (2.23), chega-se ao gradiente das velocidades conforme abaixo

$$
\begin{aligned}
\dot{\boldsymbol{F}} & =\frac{\partial \boldsymbol{F}}{\partial \boldsymbol{t}}=\frac{\partial \dot{\boldsymbol{\delta}}}{\partial \boldsymbol{\xi}} \\
& =\boldsymbol{\Omega} \boldsymbol{F}+\boldsymbol{Q}\left\{\psi_{, \alpha} \dot{p} \boldsymbol{e}_{3}^{r} \otimes \boldsymbol{e}_{\alpha}^{r}+\left[\dot{\boldsymbol{\eta}}^{r}+\dot{\boldsymbol{\kappa}}^{r} \times\left(\boldsymbol{a}^{r}+\psi p \boldsymbol{e}_{3}^{r}\right)+\psi \dot{p}^{\prime} \boldsymbol{e}_{3}^{r}+\psi \dot{p} \boldsymbol{\kappa}^{r} \times \boldsymbol{e}_{3}^{r}\right] \otimes \boldsymbol{e}_{3}^{r}\right\},
\end{aligned}
$$

onde

$$
\begin{aligned}
& \dot{\boldsymbol{\eta}}^{r}=\boldsymbol{Q}^{T}\left(\dot{\boldsymbol{u}}^{\prime}-\boldsymbol{\omega} \times \boldsymbol{z}^{\prime}\right)=\boldsymbol{Q}^{T}\left[\dot{\boldsymbol{u}}^{\prime}+\boldsymbol{z}^{\prime} \times(\boldsymbol{\Gamma} \dot{\boldsymbol{\theta}})\right] \quad \mathrm{e} \\
& \dot{\boldsymbol{\kappa}}^{r}=\boldsymbol{Q}^{T}(\dot{\boldsymbol{\kappa}}-\boldsymbol{\omega} \times \boldsymbol{\kappa})=\boldsymbol{Q}^{T} \boldsymbol{\omega}^{\prime}=\boldsymbol{Q}^{T}\left(\boldsymbol{\Gamma} \dot{\boldsymbol{\theta}}+\boldsymbol{\Gamma} \dot{\boldsymbol{\theta}}^{\prime}\right) .
\end{aligned}
$$

Por diferenciação de (2.19), obtém-se o tensor $\boldsymbol{\Gamma}^{\prime}$ que aparece em (2.25): 


$$
\begin{aligned}
\boldsymbol{\Gamma}^{\prime}= & \frac{1}{\theta^{2}}\left(\frac{\operatorname{sen} \theta}{\theta}-\frac{\operatorname{sen}^{2}(\theta / 2)}{(\theta / 2)^{2}}\right)\left(\boldsymbol{\theta} \cdot \boldsymbol{\theta}^{\prime}\right) \boldsymbol{\Theta}+ \\
& +\frac{1}{\theta^{2}}\left[\frac{1}{2} \frac{\operatorname{sen}^{2}(\theta / 2)}{(\theta / 2)^{2}}-3\left(1-\frac{\operatorname{sen} \theta}{\theta}\right) \frac{1}{\theta^{2}}\right]\left(\boldsymbol{\theta} \cdot \boldsymbol{\theta}^{\prime}\right) \boldsymbol{\Theta}^{2} \\
& +\frac{1}{2} \frac{\operatorname{sen}^{2}(\theta / 2)}{(\theta / 2)^{2}} \boldsymbol{\Theta}^{\prime}+\left(1-\frac{\operatorname{sen} \theta}{\theta}\right) \frac{1}{\theta^{2}}\left(\boldsymbol{\Theta} \boldsymbol{\Theta}^{\prime}+\boldsymbol{\Theta}^{\prime} \boldsymbol{\Theta}\right) .
\end{aligned}
$$

O vetor das deformações generalizadas da barra é definido por

$$
\boldsymbol{\varepsilon}^{r}=\left[\begin{array}{c}
\boldsymbol{\eta}^{r} \\
\boldsymbol{\kappa}^{r} \\
p \\
p^{\prime}
\end{array}\right]
$$

e o vetor dos os graus de liberdade da barra por

$$
\boldsymbol{d}=\left[\begin{array}{l}
\boldsymbol{u} \\
\boldsymbol{\theta} \\
p
\end{array}\right],
$$

Com a ajuda do operador

$$
\boldsymbol{B}=\left[\begin{array}{cccc}
\boldsymbol{Q}^{T} & \boldsymbol{O} & \boldsymbol{o} & \boldsymbol{o} \\
\boldsymbol{O} & \boldsymbol{Q}^{T} & \boldsymbol{o} & \boldsymbol{o} \\
\boldsymbol{o}^{T} & \boldsymbol{o}^{T} & 1 & 0 \\
\boldsymbol{o}^{T} & \boldsymbol{o}^{T} & 0 & 1
\end{array}\right]\left[\begin{array}{ccccc}
\boldsymbol{I} & \boldsymbol{Z}^{\prime} \boldsymbol{\Gamma} & \boldsymbol{O} & \boldsymbol{o} & \boldsymbol{o} \\
\boldsymbol{O} & \boldsymbol{\Gamma}^{\prime} & \boldsymbol{\Gamma} & \boldsymbol{o} & \boldsymbol{o} \\
\boldsymbol{o}^{T} & \boldsymbol{o}^{T} & \boldsymbol{o}^{T} & 1 & 0 \\
\boldsymbol{o}^{T} & \boldsymbol{o}^{T} & \boldsymbol{o}^{T} & 0 & 1
\end{array}\right]
$$

onde $\boldsymbol{O}$ é o tensor nulo e $Z^{\prime}$ é o tensor antissimétrico cujo vetor axial é $z$, e do operador diferencial 


$$
\boldsymbol{\Delta}=\left[\begin{array}{ccc}
\boldsymbol{I} \frac{\partial}{\partial \zeta} & \boldsymbol{O} & \boldsymbol{o} \\
\boldsymbol{O} & \boldsymbol{I} & \boldsymbol{o} \\
\boldsymbol{O} & \boldsymbol{I} \frac{\partial}{\partial \zeta} & \boldsymbol{o} \\
\boldsymbol{o}^{T} & \boldsymbol{o}^{T} & 1 \\
\boldsymbol{o}^{T} & \boldsymbol{o}^{T} & \frac{\partial}{\partial \zeta}
\end{array}\right]
$$

pode-se escrever a seguinte relação a partir de (2.25), (2.27) e (2.28):

$$
\dot{\varepsilon}^{r}=\boldsymbol{B} \boldsymbol{\Delta} \dot{\boldsymbol{d}}
$$

Os vetores de deformação $\boldsymbol{\gamma}^{r}, \boldsymbol{\eta}^{r}$ e $\boldsymbol{\kappa}^{r}$ não estão relacionados com os componentes dos tensores de deformação usualmente apresentados nas teorias de sólidos ou estruturas como por exemplo o Tensor das Deformações Lineares.

As deformações aqui apresentadas não pertencem a nenhuma família de deformações [32], mas possuem a grande vantagem de serem invariantes perante movimentos superpostos de corpo rígido.

\subsubsection{Tensões}

O tensor das tensões usado na análise não-linear para se fundamentar a formulação variacional deve estar de acordo com os Princípios da Mecânica dos Sólidos Deformáveis. O tensor das tensões empregado neste trabalho é o primeiro tensor das tensões de PiolaKirchhoff $\boldsymbol{P}$, que é energicamente conjugado com o gradiente das velocidades $\dot{\boldsymbol{F}}$, representando as tensões nominais atuantes na barra.

Através de um produto tensorial, $\boldsymbol{P}$ pode ser expresso em função de seus vetores-colunas por

$$
\boldsymbol{P}=\boldsymbol{\tau}_{\alpha} \otimes \boldsymbol{e}_{\alpha}^{r}+\boldsymbol{\tau}_{3} \otimes \boldsymbol{e}_{3}^{r}
$$

com $\tau_{\alpha}$ sendo as tensões atuantes nos planos cujas normais na configuração de referência são $\boldsymbol{e}_{\alpha}^{r}$, e $\boldsymbol{\tau}_{3}$ é a tensão atuante no plano da seção transversal por unidade de área da configuração de referência. $\tau_{\alpha}$ e $\tau_{3}$ podem ser representados também por 


$$
\begin{aligned}
\boldsymbol{\tau}_{\alpha} & =\boldsymbol{P} \boldsymbol{e}_{\alpha}^{r} \\
\boldsymbol{\tau}_{3} & =\boldsymbol{P} \boldsymbol{e}_{3}^{r},
\end{aligned}
$$

ou, na base rotacionada de versores, por

$$
\begin{aligned}
& \boldsymbol{\tau}_{\alpha}=\boldsymbol{\tau}_{\alpha \beta} \boldsymbol{e}_{\alpha}+\boldsymbol{\tau}_{\alpha 3} \boldsymbol{e}_{3} \\
& \boldsymbol{\tau}_{3}=\boldsymbol{\tau}_{3 \alpha} \boldsymbol{e}_{\alpha}+\boldsymbol{\tau}_{33} \boldsymbol{e}_{3}
\end{aligned}
$$

Nesse caso $\tau_{3 \alpha}$ e $\tau_{33}$, são, respectivamente, as tensões de cisalhamento e a tensão normal atuantes na seção transversal deformada.

\subsubsection{Potência dos Esforços Internos}

A potência dos esforços internos de uma barra de comprimento inicial $l$ e seção transversal de área A é dada por

$$
P_{\mathrm{int}}=\int_{0}^{l} \int_{A} \boldsymbol{P}: \dot{\boldsymbol{F}} d A d \zeta
$$

em que $\boldsymbol{P}$ é o primeiro tensor das tensões de Piola-Kirchhoff.

Com a ajuda de (2.24), tem-se

$$
\boldsymbol{P}: \dot{\boldsymbol{F}}=\boldsymbol{P}: \boldsymbol{\Omega} \boldsymbol{F}+\boldsymbol{P}: \boldsymbol{Q}\left\{\psi_{, \alpha} \dot{p} \boldsymbol{e}_{3}^{r} \otimes \boldsymbol{e}_{\alpha}^{r}+\left[\dot{\boldsymbol{\eta}}^{r}+\dot{\boldsymbol{\kappa}}^{r} \times\left(\boldsymbol{a}^{r}+\psi p \boldsymbol{e}_{3}^{r}\right)+\psi \dot{p}^{\prime} \boldsymbol{e}_{3}^{r}+\psi \dot{p} \boldsymbol{\kappa}^{r} \times \boldsymbol{e}_{3}^{r}\right] \otimes \boldsymbol{e}_{3}^{r}\right\}
$$

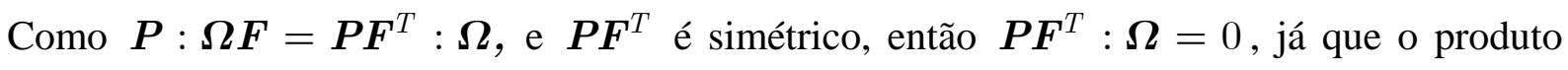
escalar de um tensor simétrico por um antissimétrico é nulo. Desta forma, com o auxílio da definição de operador transposto e de (2.32), chega-se a

$$
\begin{aligned}
\boldsymbol{P}: \dot{\boldsymbol{F}} & =\boldsymbol{P}: \boldsymbol{Q}\left\{\psi_{, \alpha} \dot{p} \boldsymbol{e}_{3}^{r} \otimes \boldsymbol{e}_{\alpha}^{r}+\left[\dot{\boldsymbol{\eta}}^{r}+\dot{\boldsymbol{\kappa}}^{r} \times\left(\boldsymbol{a}^{r}+\psi p \boldsymbol{e}_{3}^{r}\right)+\psi \dot{p}^{\prime} \boldsymbol{e}_{3}^{r}+\psi \dot{p} \boldsymbol{\kappa}^{r} \times \boldsymbol{e}_{3}^{r}\right] \otimes \boldsymbol{e}_{3}^{r}\right\} \\
& =\boldsymbol{Q}^{T} \boldsymbol{P}:\left\{\psi_{, \alpha} \dot{p} \boldsymbol{e}_{3}^{r} \otimes \boldsymbol{e}_{\alpha}^{r}+\left[\dot{\boldsymbol{\eta}}^{r}+\dot{\boldsymbol{\kappa}}^{r} \times\left(\boldsymbol{a}^{r}+\psi p \boldsymbol{e}_{3}^{r}\right)+\psi \dot{p}^{\prime} \boldsymbol{e}_{3}^{r}+\psi \dot{p} \boldsymbol{\kappa}^{r} \times \boldsymbol{e}_{3}^{r}\right] \otimes \boldsymbol{e}_{3}^{r}\right\} \\
& =\left(\boldsymbol{Q}^{T} \boldsymbol{\tau}_{\alpha}\right) \cdot \psi_{, \alpha} \dot{p} \boldsymbol{e}_{3}^{r}+\left(\boldsymbol{Q}^{T} \boldsymbol{\tau}_{3}\right) \cdot\left[\dot{\boldsymbol{\eta}}^{r}+\dot{\boldsymbol{\kappa}}^{r} \times\left(\boldsymbol{a}^{r}+\psi p \boldsymbol{e}_{3}^{r}\right)+\psi \dot{p} \boldsymbol{e}_{3}^{r}+\psi \dot{p} \boldsymbol{\kappa}^{r} \times \boldsymbol{e}_{3}^{r}\right] .
\end{aligned}
$$

A expressão acima sugere a definição dos vetores 


$$
\begin{aligned}
& \boldsymbol{\tau}_{\alpha}^{r}=\boldsymbol{Q}^{T} \boldsymbol{\tau}_{\alpha}=\boldsymbol{\tau}_{\alpha \beta} \boldsymbol{e}_{\alpha}^{r}+\boldsymbol{\tau}_{\alpha 3} \boldsymbol{e}_{3}^{r} \\
& \boldsymbol{\tau}_{3}^{r}=\boldsymbol{Q}^{T} \boldsymbol{\tau}_{3}=\boldsymbol{\tau}_{3 \alpha} \boldsymbol{e}_{\alpha}^{r}+\boldsymbol{\tau}_{33} \boldsymbol{e}_{3}^{r}
\end{aligned}
$$

Substituindo em (2.37), chega-se a

$$
\boldsymbol{P}: \dot{\boldsymbol{F}}=\psi_{, \alpha} \dot{p} \boldsymbol{\tau}_{\alpha 3}+\boldsymbol{\tau}_{3}^{r} \cdot\left[\dot{\boldsymbol{\eta}}^{r}+\dot{\boldsymbol{\kappa}}^{r} \times\left(\boldsymbol{a}^{r}+\psi p \boldsymbol{e}_{3}^{r}\right)+\psi \dot{p}^{\prime} \boldsymbol{e}_{3}^{r}+\psi \dot{p} \boldsymbol{\kappa}^{r} \times \boldsymbol{e}_{3}^{r}\right]
$$

Substituindo-se (2.39) na integral de (2.35), e após algumas manipulações, a potência dos esforços internos resulta em

$$
\boldsymbol{P}_{\mathrm{int}}=\int_{0}^{l}\left(\boldsymbol{n}^{r} \cdot \dot{\boldsymbol{\eta}}^{r}+\boldsymbol{m}^{r} \cdot \dot{\boldsymbol{\kappa}}^{r}+Q \dot{p}+B \dot{p}^{\prime}\right) d \zeta
$$

sendo $\boldsymbol{n}^{r}, \boldsymbol{m}^{r}, Q$ e B dados por

$$
\begin{aligned}
& \boldsymbol{n}^{r}=\int_{A} \boldsymbol{\tau}_{3}^{r} d A=V_{\alpha} \boldsymbol{e}_{\alpha}^{r}+N \boldsymbol{e}_{3}^{r} \\
& \boldsymbol{m}^{r}=\int_{A}\left(\boldsymbol{a}^{r}+\psi p \boldsymbol{e}_{3}^{r}\right) \times \boldsymbol{\tau}_{3}^{r} d A=M_{\alpha} \boldsymbol{e}_{\alpha}^{r}+T \boldsymbol{e}_{3}^{r} \\
& Q=\int_{A}\left[\boldsymbol{\tau}_{\alpha 3} \psi_{\alpha}+\boldsymbol{\tau}_{3}^{r} \cdot\left(\boldsymbol{\kappa}^{r} \times \boldsymbol{e}_{3}^{r}\right) \psi\right] d A \\
& B=\int_{A}\left(\boldsymbol{\tau}_{3}^{r} \cdot \boldsymbol{e}_{3}^{r}\right) \psi d A=\int_{A} \boldsymbol{\tau}_{33} \psi d A .
\end{aligned}
$$

Nota-se assim que as forças e os momentos que realmente atuam numa seção transversal são dados por

$$
\begin{aligned}
& \boldsymbol{n}=\int_{A} \boldsymbol{\tau}_{3} d A=\boldsymbol{Q} \boldsymbol{n}^{r}=V_{\alpha} \boldsymbol{e}_{\alpha}+N \boldsymbol{e}_{3} \\
& \boldsymbol{m}=\int_{A}\left(\boldsymbol{a}+\psi p \boldsymbol{e}_{3}\right) \times \boldsymbol{\tau}_{3} d A=\boldsymbol{Q} \boldsymbol{m}^{r}=M_{\alpha} \boldsymbol{e}_{\alpha}+T \boldsymbol{e}_{3} .
\end{aligned}
$$

Mesmo com esta diferença, as componentes de $\boldsymbol{n}, \boldsymbol{n}^{r}, \boldsymbol{m}$ e $\boldsymbol{m}^{r}$, respectivamente, correspondem exatamente às forças cortantes $\left(V_{\alpha}\right)$, à força normal $(N)$, aos momentos fletores $\left(M_{\alpha}\right)$ e ao momento torçor $(T)$ atuantes na seção. É importante observar que $\boldsymbol{m}^{r}, \boldsymbol{n}^{r}, Q$ e $B$ não são afetados por movimentos superpostos de corpo rígido, o que não ocorre com $\boldsymbol{n}$ e $\boldsymbol{m}$, sendo portanto convenientes na formulação de equações constitutivas. 
Nota-se que um vetor $(\cdot)^{r}$ tem na base fixa os mesmos componentes que o vetor $(\cdot)$ tem na base móvel.

Definindo-se, agora, o vetor dos esforços generalizados de uma seção

$$
\boldsymbol{\sigma}^{r}=\left[\begin{array}{c}
\boldsymbol{n}^{r} \\
\boldsymbol{m}^{r} \\
Q \\
B
\end{array}\right],
$$

com a ajuda de (2.31), a potência dos esforços internos pode ser expressa por

$$
P_{i n t}=\int_{0}^{l} \boldsymbol{\sigma}^{r} \cdot \dot{\boldsymbol{\varepsilon}}^{r} d \zeta=\int_{0}^{l} \boldsymbol{\sigma}^{r} \cdot \boldsymbol{B} \boldsymbol{\Delta} \dot{\boldsymbol{d}} d \zeta
$$

\subsubsection{Potência dos Esforços Externos}

A expressão da potência dos esforços externos em uma barra de comprimento inicial $l$ e seção transversal de área $A$ e contorno $C$, por definição, é

$$
P_{e x t}=\int_{0}^{l}\left[\int_{C} \overline{\boldsymbol{t}} \cdot \dot{\boldsymbol{\delta}} d C+\int_{A} \overline{\boldsymbol{b}} \cdot \dot{\boldsymbol{\delta}} d A\right] d \zeta
$$

sendo $\bar{t}$ o vetor das forças superficiais externas na configuração atual por unidade de área da configuração de referência e $\boldsymbol{b}$ o vetor das forças volumétricas externas na configuração atual por unidade de volume da configuração de referência, ambos atuantes na barra.

Substituindo (2.20) em (2.45), e realizando-se algumas manipulações chega-se a

$$
P_{e x t}=\int_{0}^{l}(\overline{\boldsymbol{n}} \cdot \dot{\boldsymbol{u}}+\overline{\boldsymbol{m}} \cdot \boldsymbol{\omega}+\bar{B} \dot{p}) d \zeta=\int_{0}^{l}[\overline{\boldsymbol{n}} \cdot \dot{\boldsymbol{u}}+\overline{\boldsymbol{m}} \cdot(\boldsymbol{\Gamma} \dot{\boldsymbol{\theta}})+\bar{B} \dot{p}] d \zeta
$$

onde $\overline{\boldsymbol{n}}, \overline{\boldsymbol{m}}$ e $\bar{B}$ são, respectivamente, as forças, momentos externos e bimomentos externos aplicados ao longo da barra por unidade de comprimento de referência dados por 


$$
\begin{aligned}
& \overline{\boldsymbol{n}}=\int_{C} \overline{\boldsymbol{t}} d C+\int_{A} \overline{\boldsymbol{b}} d A \mathrm{e} \\
& \overline{\boldsymbol{m}}=\int_{C}\left(\boldsymbol{a}+\psi p \boldsymbol{e}_{3}\right) \times \overline{\boldsymbol{t}} d C+\int_{A}\left(\boldsymbol{a}+\psi p \boldsymbol{e}_{3}\right) \times \overline{\boldsymbol{b}} d A, \\
& \bar{B}=\int_{C} \psi \overline{\boldsymbol{t}} \cdot \boldsymbol{e}_{3} d C+\int_{A} \psi \overline{\boldsymbol{b}} \cdot \boldsymbol{e}_{3} d A .
\end{aligned}
$$

\subsubsection{Equações de Equilíbrio e Condições de Contorno}

É utilizado o método variacional na formulação das equações de equilíbrio, neste caso o Teorema dos Trabalhos Virtuais. Variações das deformações são definidas por linearização consistente das deformações e são também chamadas de deformações virtuais.

Desta forma, a partir (2.25), essa linearização conduz a

$$
\begin{aligned}
& \delta \boldsymbol{\eta}^{r}=\boldsymbol{Q}^{T}\left[\delta \boldsymbol{u}^{\prime}+\boldsymbol{z}^{\prime} \times(\boldsymbol{\Gamma} \delta \boldsymbol{\theta})\right] \\
& \delta \boldsymbol{\kappa}^{r}=\boldsymbol{Q}^{T}\left(\boldsymbol{\Gamma}^{\prime} \delta \boldsymbol{\theta}+\boldsymbol{\Gamma} \delta \boldsymbol{\theta}^{\prime}\right)
\end{aligned}
$$

onde $\delta(\cdot)$ indica as grandezas virtuais. Portanto, pode-se se escrever

$$
\delta \varepsilon^{r}=\boldsymbol{B} \boldsymbol{\Delta} \delta \boldsymbol{d}
$$

A expressão do trabalho virtual dos esforços internos de uma barra, sendo obtida de maneira análoga à da potência interna, é então dada pela expressão abaixo

$$
\delta W_{\mathrm{int}}=\int_{0}^{l} \boldsymbol{\sigma} \cdot \delta \boldsymbol{\varepsilon}^{r} d \zeta=\int_{0}^{l}\left(\boldsymbol{n}^{r} \cdot \delta \boldsymbol{\eta}^{r}+\boldsymbol{m}^{r} \cdot \delta \boldsymbol{\kappa}^{r}+Q \delta p+B \delta p^{\prime}\right) d \zeta
$$

Já o trabalho virtual dos esforços externos aplicados ao longo da uma barra é da mesma forma dado por

$$
\delta W_{e x t}=\int_{0}^{l} \overline{\boldsymbol{q}} \cdot \delta \boldsymbol{d} d \zeta=\int_{0}^{l}[\overline{\boldsymbol{n}} \cdot \delta \boldsymbol{u}+\overline{\boldsymbol{m}} \cdot(\boldsymbol{\Gamma} \delta \boldsymbol{\theta})+\bar{B} \delta p] d \zeta
$$

sendo 


$$
\overline{\boldsymbol{q}}=\left[\begin{array}{c}
\bar{n} \\
\bar{m} \\
\bar{B}
\end{array}\right]
$$

o vetor dos esforços externos aplicados ao longo da barra.

De acordo com as relações que expressam $\delta W_{\text {int }}$ e $\delta W_{\text {ext }}$, o equilíbrio da barra com condições de contornos essenciais em suas extremidades pode ser formulado através do Teorema dos Trabalhos Virtuais, ou seja,

$$
\begin{aligned}
& \delta W_{\mathrm{int}}-\delta W_{\text {ext }}=0, \forall \delta \boldsymbol{d}=\delta \hat{\boldsymbol{d}}(\zeta) \mid \delta \hat{\boldsymbol{d}}(0)=\delta \hat{\boldsymbol{d}}(l)=\boldsymbol{o} \Rightarrow \\
& \int_{0}^{l}\left[\boldsymbol{n} \cdot \delta \boldsymbol{u}^{\prime}+\boldsymbol{m} \cdot(\boldsymbol{\Gamma} \delta \boldsymbol{\theta})^{\prime}-\left(\boldsymbol{z}^{\prime} \times \boldsymbol{n}\right) \cdot(\boldsymbol{\Gamma} \delta \boldsymbol{\theta})+Q \delta p+B \delta p^{\prime}-\overline{\boldsymbol{n}} \cdot \delta \boldsymbol{u}-\overline{\boldsymbol{m}} \cdot(\boldsymbol{\Gamma} \delta \boldsymbol{\theta})-\bar{B} \delta p\right] d \zeta=0 .
\end{aligned}
$$

Efetuando-se integração por partes nos termos com $\delta \boldsymbol{u}^{\prime}$ e $(\boldsymbol{\Gamma} \delta \boldsymbol{\theta})^{\prime}$, chega-se à seguinte equação

$$
\int_{0}^{l}\left[\left(\boldsymbol{n}^{\prime}+\overline{\boldsymbol{n}}\right) \cdot \delta \boldsymbol{u}+\left(\boldsymbol{m}^{\prime}+\boldsymbol{z}^{\prime} \times \boldsymbol{n}+\overline{\boldsymbol{m}}\right) \cdot(\boldsymbol{\Gamma} \delta \boldsymbol{\theta})+\left(B^{\prime}-Q+\bar{B}\right) \delta p\right] d \zeta=0, \forall \delta \boldsymbol{d} . \text { (2.54) }
$$

Para a igualdade acima ser válida, ela deve conduzir às seguintes condições de contorno:

$$
\begin{aligned}
& \left.\left(\boldsymbol{n}-\boldsymbol{n}^{*}\right) \cdot \delta \boldsymbol{u}\right|_{0} ^{l}=0, \\
& \left.\left(\boldsymbol{\Gamma}^{T} \boldsymbol{m}-\boldsymbol{\mu}^{*}\right) \cdot \delta \boldsymbol{\theta}\right|_{0} ^{l}=0, \\
& \left.\left(B-B^{*}\right) \delta p\right|_{0} ^{l}=0 .
\end{aligned}
$$

As condições de contornos naturais que anulam os termos de contorno são

$$
\begin{array}{rlllrl}
\boldsymbol{n}(0) & =\boldsymbol{n}^{*}(0) & \text { e } & \boldsymbol{n}(l) & =\boldsymbol{n}^{*}(l), \\
\boldsymbol{\Gamma}^{T} \boldsymbol{m}(0) & =\boldsymbol{\mu}^{*}(0) & \text { e } & \boldsymbol{\Gamma}^{T} \boldsymbol{m}(l) & =\boldsymbol{\mu}^{*}(l), \\
B(0) & =B^{*}(0) & \text { e } & & B(l) & =B^{*}(l) .
\end{array}
$$

e as seguintes condições de contorno essenciais: 


$$
\begin{aligned}
& \boldsymbol{u}(0)=\boldsymbol{u}^{*}(0) \text { e } \boldsymbol{u}(l)=\boldsymbol{u}^{*}(l), \\
& \boldsymbol{\theta}(0)=\boldsymbol{\theta}^{*}(0) \text { e } \boldsymbol{\theta}(l)=\boldsymbol{\theta}^{*}(l) \text {, } \\
& p(0)=p^{*}(0) \text { e } p(l)=p^{*}(l) \text {. }
\end{aligned}
$$

\subsubsection{Linearização dos trabalhos virtuais: operador Tangente}

A aplicação do Método dos Elementos Finitos em estruturas não lineares recai na utilização do método de Newton para solução do sistema, o qual invariavelmente necessita da linearização das expressões que definem o equilíbrio do sistema. Essa linearização pode ser realizada através da derivada de Fréchet resultando no chamado operador tangente. Assim diferenciando (2.53) no tempo e usando (2.49) e (2.50), tem-se

$$
\frac{\partial}{\partial t}\left(\delta W_{\mathrm{int}}-\delta W_{\text {ext }}\right)=\int_{0}^{l}\left(\dot{\boldsymbol{\sigma}}^{r} \cdot \boldsymbol{B} \boldsymbol{\Delta} \delta \boldsymbol{d}+\boldsymbol{\sigma}^{r} \cdot \dot{\boldsymbol{B}} \boldsymbol{\Delta} \delta \boldsymbol{d}-\dot{\overline{\boldsymbol{q}}} \cdot \delta \boldsymbol{d}\right) d \zeta
$$

Depois de muitas manipulações algébricas, de (2.58) chega-se a

$$
\frac{\partial}{\partial t}\left(\delta W_{\mathrm{int}}-\delta W_{e x t}\right)=\int_{0}^{l}[(\boldsymbol{D} \boldsymbol{B} \boldsymbol{\Delta} \dot{\boldsymbol{d}}) \cdot(\boldsymbol{B} \boldsymbol{\Delta} \delta \boldsymbol{d})+(\boldsymbol{G} \boldsymbol{\Delta} \dot{\boldsymbol{d}}) \cdot(\boldsymbol{\Delta} \delta \boldsymbol{d})-(\boldsymbol{L} \dot{\boldsymbol{d}}) \cdot \delta \boldsymbol{d}] d \zeta
$$

onde

$$
\boldsymbol{D}=\frac{\partial \boldsymbol{\sigma}^{r}}{\partial \boldsymbol{\varepsilon}^{r}}=\left[\begin{array}{cccc}
\frac{\partial \boldsymbol{n}^{r}}{\partial \boldsymbol{\eta}^{r}} & \frac{\partial \boldsymbol{n}^{r}}{\partial \boldsymbol{k}^{r}} & \frac{\partial \boldsymbol{n}^{r}}{\partial p} & \frac{\partial \boldsymbol{n}^{r}}{\partial p^{\prime}} \\
\frac{\partial \boldsymbol{m}^{r}}{\partial \boldsymbol{\eta}^{r}} & \frac{\partial m^{r}}{\partial \kappa^{r}} & \frac{\partial m^{r}}{\partial p} & \frac{\partial m^{r}}{\partial p^{\prime}} \\
\frac{\partial Q}{\partial \boldsymbol{\eta}^{r}} & \frac{\partial Q}{\partial \boldsymbol{\kappa}^{r}} & \frac{\partial Q}{\partial p} & \frac{\partial Q}{\partial p^{\prime}} \\
\frac{\partial B}{\partial \boldsymbol{\eta}^{r}} & \frac{\partial B}{\partial \boldsymbol{k}^{r}} & \frac{\partial B}{\partial p} & \frac{\partial B}{\partial p^{\prime}}
\end{array}\right]
$$

é a matriz dos módulos constitutivos de rigidez tangente 


$$
\boldsymbol{G}=\left[\begin{array}{ccccc}
\boldsymbol{O} & \boldsymbol{G}_{u^{\prime} \theta} & \boldsymbol{O} & \boldsymbol{o} & \boldsymbol{o} \\
\boldsymbol{G}_{u^{\prime} \theta}^{T} & \boldsymbol{G}_{\theta \theta} & \boldsymbol{G}_{\theta \theta^{\prime}} & \boldsymbol{o} & \boldsymbol{o} \\
\boldsymbol{O} & \boldsymbol{G}_{\theta \theta^{\prime}}^{T} & \boldsymbol{O} & \boldsymbol{o} & \boldsymbol{o} \\
\boldsymbol{o}^{T} & \boldsymbol{o}^{T} & \boldsymbol{o}^{T} & 0 & 0 \\
\boldsymbol{o}^{T} & \boldsymbol{o}^{T} & \boldsymbol{o}^{T} & 0 & 0
\end{array}\right]
$$

é a matriz simétrica que caracteriza os efeitos geométricos dos esforços internos e

$$
\boldsymbol{L}=\frac{\partial \overline{\boldsymbol{q}}}{\partial \boldsymbol{d}}=\left[\begin{array}{ccc}
\boldsymbol{O} & \boldsymbol{O} & \boldsymbol{o} \\
\boldsymbol{O} & \boldsymbol{L}_{\theta \theta} & \boldsymbol{L}_{\theta p} \\
\boldsymbol{o}^{T} & \boldsymbol{L}_{\theta p}^{T} & 0
\end{array}\right]
$$

é a matriz que caracteriza os efeitos geométricos dos esforços externos.

As submatrizes de $\boldsymbol{D}$ podem ser obtidas desde que se conheçam $\boldsymbol{n}^{r}, \boldsymbol{m}^{r}, Q$ e $B$ (dados por (2.41)), isto é, desde que se conheça a equação constitutiva, a qual fornece $\boldsymbol{\tau}_{\alpha}^{r}$ e $\boldsymbol{\tau}_{3}^{r}$. Já as submatrizes $\boldsymbol{G}$ e $\boldsymbol{L}$, a partir da ajuda dos tensores antissimétricos $\boldsymbol{N}, \boldsymbol{M}$ e $\boldsymbol{Z}^{\prime}$ (cujos vetores axiais são $\boldsymbol{n}, \boldsymbol{m}$ e $\boldsymbol{z}^{\prime}$, respectivamente) são dadas por

$$
\begin{aligned}
& \boldsymbol{G}_{u^{\prime} \theta}=-\boldsymbol{N} \boldsymbol{\Gamma} \\
& \boldsymbol{G}_{u^{\prime} \theta}^{T}=\boldsymbol{\Gamma}^{T} \boldsymbol{N} \\
& \boldsymbol{G}_{\theta \theta}=\frac{1}{2} \boldsymbol{\Gamma}^{T}\left(\boldsymbol{Z}^{\prime} \boldsymbol{N}+\boldsymbol{N} \boldsymbol{Z}^{\prime}\right) \boldsymbol{\Gamma}-\boldsymbol{U}\left(\boldsymbol{\theta}, \boldsymbol{Z}^{\prime} \boldsymbol{n}\right)+\boldsymbol{U}^{\prime}\left(\boldsymbol{\theta}^{\prime}, \boldsymbol{\theta}, \boldsymbol{m}\right)-\frac{1}{2}\left(\boldsymbol{\Gamma}^{T^{\prime}} \boldsymbol{M} \boldsymbol{\Gamma}-\boldsymbol{\Gamma}^{T} \boldsymbol{M} \boldsymbol{\Gamma}^{\prime}\right) \\
& \boldsymbol{G}_{\theta \theta}{ }^{\prime}=\boldsymbol{U}(\boldsymbol{\theta}, \boldsymbol{m})+\frac{1}{2} \boldsymbol{\Gamma}^{T} \boldsymbol{M} \boldsymbol{\Gamma}=\boldsymbol{V}(\boldsymbol{\theta}, \boldsymbol{m}) \\
& \boldsymbol{G}_{\theta \theta^{\prime}}^{T}=\boldsymbol{U}(\boldsymbol{\theta}, \boldsymbol{m})+\frac{1}{2} \boldsymbol{\Gamma}^{T} \boldsymbol{M} \boldsymbol{\Gamma}=\boldsymbol{V}^{T}(\boldsymbol{\theta}, \boldsymbol{m})
\end{aligned}
$$

e

$$
\begin{array}{rll}
\boldsymbol{L}_{u u}=\frac{\partial \overline{\boldsymbol{n}}}{\partial \boldsymbol{u}} & \boldsymbol{L}_{\theta u}=\frac{\partial\left(\boldsymbol{\Gamma}^{T} \overline{\boldsymbol{m}}\right)}{\partial \boldsymbol{u}} & \boldsymbol{L}_{p u}=\frac{\partial \bar{B}}{\partial \boldsymbol{u}} \\
\boldsymbol{L}_{u \theta}=\frac{\partial \overline{\boldsymbol{n}}}{\partial \boldsymbol{\theta}} & \boldsymbol{L}_{\theta \theta}=\frac{\partial\left(\boldsymbol{\Gamma}^{T} \overline{\boldsymbol{m}}\right)}{\partial \boldsymbol{\theta}} & \boldsymbol{L}_{p \theta}=\frac{\partial \bar{B}}{\partial \boldsymbol{\theta}} \\
\boldsymbol{L}_{u p}=\frac{\partial \overline{\boldsymbol{n}}}{\partial p} & \boldsymbol{L}_{\theta \theta}=\frac{\partial\left(\boldsymbol{\Gamma}^{T} \overline{\boldsymbol{m}}\right)}{\partial p} & \boldsymbol{L}_{p p}=\frac{\partial \bar{B}}{\partial p}
\end{array}
$$


O tensor $\boldsymbol{L}$ depende diretamente dos esforços externos atuantes na barra. Sendo esses não conservativos, $\boldsymbol{L}$ não será simétrica.

Sendo a matriz constitutiva $\boldsymbol{D}$ simétrica e os carregamentos ao longo da barra sendo conservativos, o operador tangente sempre será simétrico, uma vez que as matrizes $\boldsymbol{G} e \boldsymbol{L}$ são simétricas. Cabe mencionar que as expressões (2.63) foram publicadas de forma correta pela primeira vez na literatura em [19], corrigindo o erro cometido por Simo em [20].

\subsection{Implementação em método dos elementos finitos}

O Método dos Elementos Finitos (MEF) é uma ferramenta muito utilizada na solução de problemas da Mecânica dos Sólidos Deformáveis, sendo possível fazer a análise de sistemas estruturais bastante complexos, com condições de vínculo arbitrárias e carregamentos bem genéricos.

Este item a aplicação do MEF para a resolução da equação (2.53). Os elementos utilizados serão elementos de barra isoparamétricos de 2 e 3 nós.

\subsubsection{Formulação matricial}

Cada nó de um elemento possui sete graus de liberdade, três translações, três rotações e um parâmetro de empenamento. Os deslocamentos $\boldsymbol{d}$ para toda a estrutura serão obtidos através da interpolação dos valores nodais. Os valores interpolados serão introduzidos nas expressões dos trabalhos virtuais e de suas linearizações, dando origem às equações das forças residuais e às matrizes de rigidez tangente constitutiva, geométrica e de carregamento. Com essas últimas, resolver-se-ão os sistemas não lineares resultantes, através do Método de Newton.

$\mathrm{Na}$ formulação do Método dos Elementos Finitos, interpolam-se os deslocamentos generalizados $\boldsymbol{d}$ das seções transversais de um elemento de barra utilizando a relação:

$$
d=\mathbf{N p},
$$


onde $\boldsymbol{N}=\hat{\boldsymbol{N}}(\xi)$ é a matriz que contém as funções de interpolação e $\boldsymbol{p}$ é o vetor dos deslocamentos nodais generalizados do elemento, dado por

$$
\mathbf{p}=\left[\begin{array}{c}
\boldsymbol{p}_{1} \\
\boldsymbol{p}_{2} \\
\boldsymbol{p}_{3} \\
\vdots \\
\boldsymbol{p}_{n}
\end{array}\right]
$$

( $\boldsymbol{p}_{i}$ representa o vetor dos graus de liberdade do nó $i$ e $n$ é o número de nós do elemento).

Os componentes da matriz de interpolação $\mathbf{N}$ nada mais são do que as funções de forma dos elementos isoparamétricos, ou seja, polinômios de Lagrange [8], [33] do tipo

$$
N_{a}=l_{a}^{n-1}(\xi)=\frac{\left(\xi-\xi_{1}\right)\left(\xi-\xi_{2}\right) \ldots\left(\xi-\xi_{a-1}\right)\left(\xi-\xi_{a+1}\right) \ldots\left(\xi-\xi_{n}\right)}{\left(\xi_{a}-\xi_{1}\right)\left(\xi_{a}-\xi_{2}\right) \ldots\left(\xi_{a}-\xi_{a-1}\right)\left(\xi_{a}-\xi_{a+1}\right) \ldots\left(\xi_{a}-\xi_{n}\right)}
$$

As funções de forma do elemento de barra com $n$ nós podem ser expressas pela equação

$$
\mathbf{N}_{a}=l_{a}^{n-1} \boldsymbol{I}_{p}=N_{a} \boldsymbol{I}_{p}
$$

onde $\boldsymbol{I}_{p}$ é uma matriz identidade com dimensão igual ao número de graus de liberdade por nó. A matriz de interpolação $\mathbf{N}$ é dada por

$$
\mathbf{N}=\left[\begin{array}{llll}
\boldsymbol{N}_{1} & \boldsymbol{N}_{2} & \ldots & \boldsymbol{N}_{n}
\end{array}\right]
$$

As componente da matriz $N$ assumem os seguintes valores para elementos de barra de dois nós:

$$
\begin{aligned}
& N_{1}(\xi)=l_{1}^{1}(\xi)=\frac{1}{2}(1-\xi) \text { e } \\
& N_{2}(\xi)=l_{2}^{1}(\xi)=\frac{1}{2}(1+\xi) .
\end{aligned}
$$


Para elementos de três nós obtém-se:

$$
\begin{aligned}
& N_{1}(\xi)=l_{1}^{2}(\xi)=\frac{1}{2} \xi(\xi-1), \\
& N_{2}(\xi)=l_{2}^{2}(\xi)=\left(1-\xi^{2}\right) \mathrm{e} \\
& N_{3}(\xi)=l_{3}^{2}(\xi)=\frac{1}{2} \xi(1+\xi) .
\end{aligned}
$$

As coordenadas $\zeta$ da barra são válidas no intervalo de 0 a $l$, enquanto que as coordenadas $\xi$ do elemento estão normalizadas para o intervalo -1 a 1 . Como se trata de elementos isoparamétricos, a forma de contornar este fato é imediata. Para elementos de dois nós, obtém-se:

$$
\zeta=\zeta_{1} N_{1}+\zeta_{2} N_{2}=l N_{2}(\xi)=\frac{l}{2}(1+\xi)
$$

(já que as posições dos nós 1 e 2 são $\zeta_{1}=0$ e $\zeta_{2}=l$ ), de modo que o Jacobiano da transformação de coordenadas será

$$
J_{\xi}=\frac{\partial \zeta}{\partial \xi}=l \frac{\partial N_{2}(\xi)}{\partial \xi}=\frac{l}{2} .
$$

No caso do elemento de três nós, com o nó intermediário em uma posição qualquer $\alpha l$, com $0<\alpha<l$ obtemo-se:

$$
\zeta=\zeta_{1} N_{1}+\zeta_{2} N_{2}+\zeta_{3} N_{3}=\alpha l N_{2}(\xi)+l N_{3}(\xi)
$$

O jacobiano neste caso será:

$$
J_{\xi}=\frac{\partial \zeta}{\partial \xi}=\alpha l \frac{\partial N_{2}(\xi)}{\partial \xi}+l \frac{\partial N_{3}(\xi)}{\partial \xi}
$$

As derivadas das funções de forma em relação a $\zeta$, pela regra da cadeia, serão dadas por

$$
\frac{\partial N_{a}}{\partial \zeta}=\frac{\partial N_{a}}{\partial \xi} \frac{\partial \xi}{\partial \zeta}=\left(J_{\xi}\right)^{-1} \frac{\partial N_{a}}{\partial \xi},
$$

valendo para elementos com qualquer número de nós. 
De (2.65), obtém-se:

$$
\delta \boldsymbol{d}=\mathbf{N} \delta \mathbf{p}
$$

onde $\delta \mathbf{p}$ é o vetor dos deslocamentos virtuais nodais do elemento.

Substituindo os vetores $\boldsymbol{d}$ e $\delta \boldsymbol{d}$ por (2.65) e (2.77) nas expressões dos trabalhos virtuais, pode-se dizer que $\delta W=\delta W_{\text {int }}-\delta W_{\text {ext }}$ das forças residuais de um elemento é dado por

$$
\begin{aligned}
\delta W & =\int_{0}^{l}[\boldsymbol{\sigma} \cdot \boldsymbol{B} \boldsymbol{\Delta}(\mathbf{N} \delta \boldsymbol{p})-\overline{\boldsymbol{q}} \cdot(\mathbf{N} \delta \boldsymbol{p})] d \zeta-\left.\boldsymbol{q}^{*} \cdot(\mathbf{N} \delta \boldsymbol{p})\right|_{0} ^{l}= \\
& =\left(\int_{0}^{l}\left[(\boldsymbol{\Delta} \mathbf{N})^{T} \boldsymbol{B}^{T} \boldsymbol{\sigma}^{r}-\mathbf{N}^{T} \overline{\boldsymbol{q}}\right] d \zeta\right) \cdot \delta \boldsymbol{p}-\left.\left(\mathbf{N}^{T} \boldsymbol{q}^{*}\right)\right|_{0} ^{l} \cdot \delta \boldsymbol{p}= \\
& =\mathbf{P} \cdot \delta \boldsymbol{p}-\left.\left(\mathbf{N}^{T} \boldsymbol{q}^{*}\right)\right|_{0} ^{l} \cdot \delta \boldsymbol{p} .
\end{aligned}
$$

Neste caso $q^{*}$ é o vetor dos esforços concentrados atuantes nas extremidades do elemento e $\mathbf{P}$ é o vetor do esforços nodais residuais do elemento (não confundir o vetor $\mathbf{P}$ com o tensor das tensões $\boldsymbol{P}$ de Piola-Kirchhoff). A condição de equilíbrio implica que:

$$
\mathbf{P} \cdot \delta \boldsymbol{p}=\left.\left(\mathbf{N}^{T} \boldsymbol{q}^{*}\right)\right|_{0} ^{l} \cdot \delta \boldsymbol{p}
$$

Daí decorre:

$$
\begin{aligned}
\mathbf{P} & =\left.\left(\mathbf{N}^{T} \boldsymbol{q}^{*}\right)\right|_{0} ^{l} \therefore \\
\mathbf{P} & =\mathbf{N}_{2}^{T} \boldsymbol{q}^{*}(l)-\mathbf{N}_{1}^{T} \boldsymbol{q}^{*}(0)= \\
& =\int_{0}^{l}\left[(\boldsymbol{\Delta} \mathbf{N})^{T} \boldsymbol{B}^{T} \boldsymbol{\sigma}^{r}-\mathbf{N}^{T} \overline{\boldsymbol{q}}\right] d \zeta
\end{aligned}
$$

sendo necessário efetuar a mudança de coordenadas nas integrações, mediante uso do Jacobiano.

A matriz rigidez tangente do elemento decorre da introdução de (2.65) e (2.77) em (2.59), o que após alguma álgebra conduz a:

$$
\mathbf{k}_{T}=\frac{\partial \mathbf{P}}{\partial \boldsymbol{p}}
$$


Como $\mathbf{P}$ é dado em (2.80), a diferenciação acima é possível de ser realizada, de maneira que, após algumas manipulações algébricas, chega-se a

$$
\begin{aligned}
\mathbf{k}_{T} & =\int_{0}^{l}(\boldsymbol{\Delta} \mathbf{N})^{T} \boldsymbol{B}^{T} \boldsymbol{D} \boldsymbol{B}(\boldsymbol{\Delta} \mathbf{N}) d \zeta+\int_{0}^{l}(\boldsymbol{\Delta} \mathbf{N})^{T} \boldsymbol{G}(\boldsymbol{\Delta} \mathbf{N}) d \zeta-\int_{0}^{l} \mathbf{N}^{T} \boldsymbol{L} \mathbf{N} d \zeta \\
& =\mathbf{k}_{c}+\mathbf{k}_{G}-\mathbf{k}_{L} .
\end{aligned}
$$

Os tensores $\boldsymbol{G}$ e $\boldsymbol{L}$ que aparecem nas integrais acima são aqueles expressos pelas equações (2.60) e (2.61), respectivamente, que caracterizam os efeitos geométricos dos esforços internos e externos. Além disso, as parcelas $\mathbf{k}_{c}, \mathbf{k}_{G}$ e $\mathbf{k}_{L}$ são chamadas de contribuições constitutiva, geométrica e de carregamento na matriz de rigidez.

\subsubsection{Formulação das matrizes globais e solução do problema}

Seja $\mathbf{A}_{j}$ a matriz que "localiza” os nós do elemento $j$ na estrutura global. A partir dessa matriz, chamada de matriz de conectividade, o vetor $\boldsymbol{p}_{j}$ dos graus de liberdade nodais do elemento $j$ pode ser extraído do vetor $\boldsymbol{r}$ dos graus de liberdade nodais de toda a estrutura:

$$
\boldsymbol{p}_{j}=\mathbf{A}_{j} \boldsymbol{r}
$$

O vetor $\mathbf{R}$ dos esforços nodais residuais de toda a estrutura pode ser relacionado com o vetor $\mathbf{P}_{j}$ dos esforços nodais residuais do elemento $j$ :

$$
\mathbf{R}=\sum_{j=1}^{n} \mathbf{A}_{j}^{T} \mathbf{P}_{j}
$$

Observa-se que $\mathbf{R}$, sendo função de $\mathbf{P}_{j}$, é função de $\boldsymbol{p}_{j}$, devido a (2.80) e assim, como pode ser visto em (2.83), é uma função dos graus de liberdade nodais $r$ da estrutura, já que $\boldsymbol{p}_{j}=\mathbf{A}_{j} \boldsymbol{r}$

A estrutura estará em equilíbrio quando a resultante dos esforços residuais globais for nula, isto é, se existir um campo de deslocamentos e empenamentos que satisfaça:

$$
\mathbf{R}=\boldsymbol{o}
$$


A equação (2.85) resulta num sistema de equações não lineares, cuja solução pode ser obtida por exemplo através do Método de Newton [8], [33] e [34]. Assim, aplicando-se o Método de Newton, arbitra-se uma estimativa inicial para o vetor $r$ e a partir dela ter-se-á:

$$
\boldsymbol{r}^{i+1}=\boldsymbol{r}^{i}-\left(\frac{\partial \mathbf{R}}{\partial \boldsymbol{r}}\left(\boldsymbol{r}^{i}\right)\right)^{-1} \mathbf{R}\left(\boldsymbol{r}^{i}\right)
$$

sendo $\boldsymbol{r}^{i}$ e $\boldsymbol{r}^{i+1}$ os graus de liberdade nodais da estrutura nas iterações $i$ e $i+1$, respectivamente. Utilizando-se algumas expressões anteriores, a derivada parcial que surge em (2.85) fica:

$$
\begin{aligned}
\frac{\partial \mathbf{R}}{\partial \boldsymbol{r}} & =\frac{\partial}{\partial \boldsymbol{r}}\left(\sum_{j=1}^{n} \boldsymbol{A}_{j}^{T} \mathbf{P}_{j}\right) \\
& =\sum_{j=1}^{n} \frac{\partial}{\partial \boldsymbol{r}}\left(\boldsymbol{A}_{j}^{T} \mathbf{P}_{j}\right) \\
& =\sum_{j=1}^{n} \boldsymbol{A}_{j}^{T} \frac{\partial \mathbf{P}_{j}}{\partial \boldsymbol{r}} \\
& =\sum_{j=1}^{n} \boldsymbol{A}_{j}^{T} \frac{\partial \mathbf{P}_{j}}{\partial \boldsymbol{p}_{j}} \frac{\partial \boldsymbol{p}_{j}}{\partial \boldsymbol{r}} \\
& =\sum_{j=1}^{n} \boldsymbol{A}_{j}^{T} \mathbf{k}_{T j} \boldsymbol{A}_{j} \\
& =\mathbf{K}_{T},
\end{aligned}
$$

onde $\mathbf{k}_{T j}$ é a matriz de rigidez tangente do elemento $j$, expressa em (2.80) e (2.81), e $\mathbf{K}_{T}$ é a matriz de rigidez tangente da estrutura, ambas no sistema global. Então, (2.85) pode ser escrita como

$$
\boldsymbol{r}^{i+1}=\boldsymbol{r}^{i}-\left(\mathbf{K}_{T}^{i}\right)^{-1} \mathbf{R}\left(\boldsymbol{r}^{i}\right)
$$

Lembrando que para elementos formulados sob linearidade geométrica, a matriz de rigidez global fica independente dos deslocamentos, já que o equilíbrio é escrito na configuração de referência (não há contribuição geométrica e nem de carregamento em $\mathbf{k}_{T}$ ) [1]. Como consequência, o sistema de equações resultante da condição de equilíbrio é algebricamente linear, e pode ser, portanto, resolvido por meio de métodos diretos, como o de Crout [34], que verifica se a matriz é positivo definida. Sem necessidade de iterações. 


\section{Equações constitutivas elásticas}

No âmbito da elasticidade, as equações constitutivas são igualdades que relacionam o campo de tensões com o campo de deformações. São relações entre grandezas físicas que procuram introduzir em um modelo mecânico as propriedades dos materiais [32].

Este capítulo aborda a questão da formulação adequada de relações constitutivas elásticas, para aplicação na teoria de barras cinematicamente exata.

A teoria apresentada no capítulo anterior é válida para barras constituídas de qualquer tipo de material. Neste capítulo é apresentada uma extensão dos trabalhos de [2] e [25], apresentando uma equação constitutiva elástica “completa” (isto é, incluindo todos os termos de ordem superior nas deformações) para análise de barras com graus de liberdade de empenamento. O ponto de partida é o modelo constitutivo hiperelástico tridimensional de Kirchhoff-St-Venant. A título de curiosidade, apresenta-se inicialmente a formulação de uma equação constitutiva elática-linear para a teoria de barras em questão. Partindo-se em seguida para a formulação com o material de Kirchhoff-St-Venant. Como desenvolvimento futuro, apresenta-se, no final do capítulo, uma possibilidade de extensão dessas idéias para o material hiperelástico de Simo-Ciarlet [35].

\subsection{Equação constitutiva elástica linear}

Em não linearidade geométrica deve-se ressaltar a importância do Princípio da Objetividade, segundo o qual uma equação constitutiva não pode ser afetada por movimentos de corpo rígido superpostos ao movimento do sólido. Em outras palavras, como os movimentos de corpo rígido não provocam deformações, não devem alterar o estado interno de tensões do sólido em estudo [32].

Para a teoria geometricamente exata de barras no espaço, as componentes de alongamento e de distorção da deformação também podem ser relacionadas com as suas tensões correspondentes (isto é, com suas tensões energicamente conjugadas), através dos módulos de elasticidade longitudinal e transversal do material [1]. As expressões que definem estas deformações podem ser extraídas, por exemplo, do tensor de Green $\boldsymbol{E}$, que para o caso de cinemática exata vale: 


$$
\boldsymbol{E}=\frac{1}{2}(\boldsymbol{C}-\boldsymbol{I})
$$

onde $\boldsymbol{C}$ é o tensor direito de Cauchy, dado por:

$$
\boldsymbol{C}=\boldsymbol{F}^{T} \boldsymbol{F}
$$

Substituindo-se (2.15) e (3.2) em (3.1), e desenvolvendo a expressão, chega-se a:

$$
\boldsymbol{E}=\frac{1}{2}\left(\left[\begin{array}{ccc}
1 & 0 & \gamma_{1}^{r} \\
0 & 1 & \gamma_{2}^{r} \\
p \psi_{, 1} & p \psi_{, 2} & \varepsilon
\end{array}\right]^{T}\left[\begin{array}{ccc}
1 & 0 & \gamma_{1}^{r} \\
0 & 1 & \gamma_{2}^{r} \\
p \psi_{, 1} & p \psi_{, 2} & \varepsilon
\end{array}\right]-\boldsymbol{I}\right)
$$

onde $\gamma_{\alpha}^{r}$ e $\varepsilon$ são as 3 componentes do vetor $\gamma^{r}$ (dada pela equação (2.16)), isto é

$$
\boldsymbol{\gamma}^{r}=\left[\begin{array}{c}
\gamma_{1}^{r} \\
\gamma_{2}^{r} \\
\varepsilon
\end{array}\right]=\left[\begin{array}{c}
\eta_{1}^{r}-x_{2} \kappa_{3}^{r}+p \psi \kappa_{2}^{r} \\
\eta_{2}^{r}+x_{1} \kappa_{3}^{r}-p \psi \kappa_{1}^{r} \\
\eta_{3}^{r}-x_{1} \kappa_{2}^{r}+x_{2} \kappa_{1}^{r}-p^{\prime} \psi
\end{array}\right]=\gamma_{\alpha}^{r} \boldsymbol{e}_{\alpha}^{r}+\varepsilon \boldsymbol{e}_{3}^{r}
$$

Desenvolvendo as operações matriciais acima, e desprezando os todos os termos a partir da segunda ordem nas deformações, obtém-se:

$$
\boldsymbol{E}=\frac{1}{2}\left[\begin{array}{ccc}
0 & 0 & \gamma_{1}^{r}+p \psi_{, 1} \\
0 & 0 & \gamma_{1}^{r}+p \psi_{, 2} \\
\gamma_{1}^{r}+p \psi_{, 1} & \gamma_{2}^{r}+p \psi_{, 2} & 2 \varepsilon
\end{array}\right]
$$

Quando se despreza os termos de ordem superior em $\boldsymbol{E}$, implicitamente se presume que as deformações da barra são pequenas, embora os deslocamentos e as rotações possam ser de qualquer magnitude.

Como, até a primeira ordem nas deformações $\boldsymbol{Q}^{T} \boldsymbol{P}=\boldsymbol{S}$ ( $\boldsymbol{S}$ = segundo tensor das tensões de Piola-Kirchhoff), as tensões retrorrotacionadas de $\boldsymbol{P}$ podem ser relacionadas com as deformações $\boldsymbol{E}$ de Green uma vez que são essas últimas as deformações energeticamente conjugadas a $S$. 
Dessa forma, a partir de (3.5), a lei elástica linear clássica envolvendo todas as distorções e todos os alongamentos da barra estabelece que

$$
\begin{aligned}
\tau_{33} & =E \varepsilon \\
\tau_{3 \alpha} & =G\left(\gamma_{\alpha}^{r}+p \psi_{, \alpha}\right) \\
\tau_{\alpha 3} & =G\left(\gamma_{\alpha}^{r}+p \psi_{, \alpha}\right),
\end{aligned}
$$

onde as tensões $\tau_{33}, \tau_{\alpha 3}$ e $\tau_{3 \alpha}$ são definidas em (2.34). Sendo o vetor $\boldsymbol{\sigma}^{r}$ objetivo, então as relações de (3.6) podem ser aplicadas nas suas componentes, ou seja, nas expressões que definem $V_{\alpha}, N, M_{\alpha}, T, Q$ e $B$, de onde resultam diversas equações relacionando os esforços internos e as deformações generalizadas [1].

Produtos de deformação do tipo $\eta_{i} \kappa_{i}, \eta_{i} p, \kappa_{i} p, \eta_{i} p^{\prime} \quad\left(\eta_{i}=\right.$ componentes de $\boldsymbol{\eta}^{r} ; \kappa_{i}=$ componentes de $\boldsymbol{\kappa}^{r}$ ) e aparecem nestas expressões, devendo ser desprezados mais uma vez já que as deformações são consideradas pequenas. Após essa simplificação, pode-se escrever matricialmente que

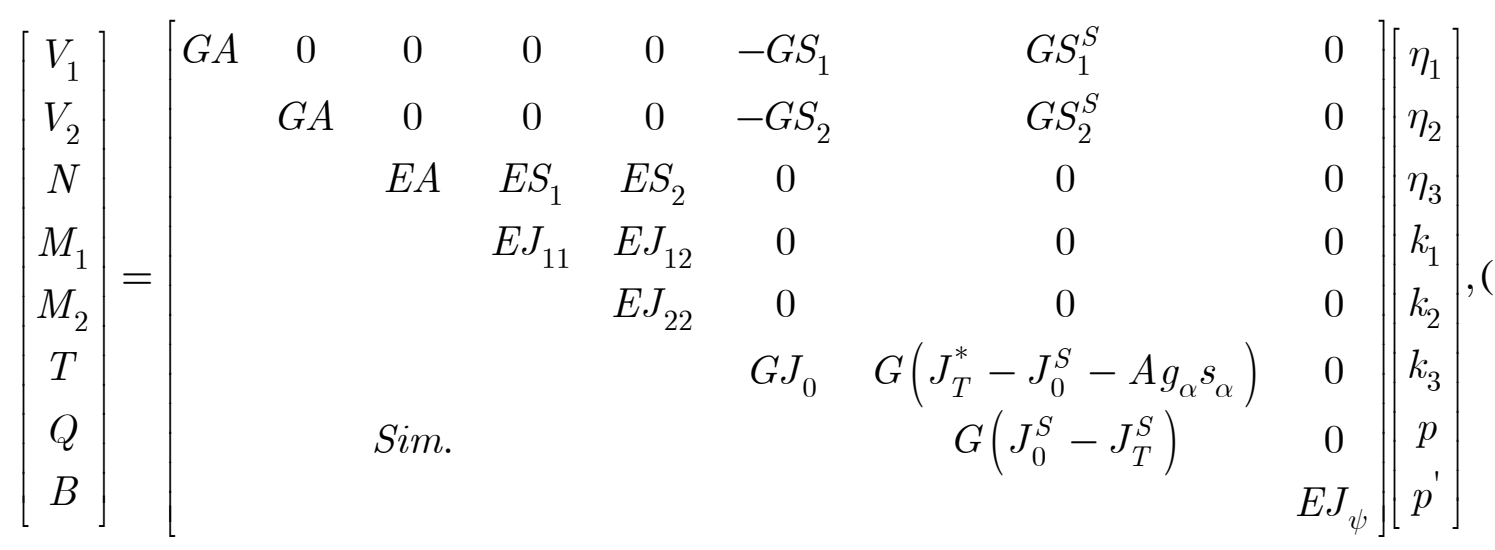

ou

$$
\boldsymbol{\sigma}^{r}=\boldsymbol{D} \varepsilon^{r}
$$

$D$ é a chamada matriz dos módulos de rigidez constitutiva, no sistema local da barra.

Conforme apontado em [1], desprezar os termos de segunda ordem nas deformações faz com que a matriz dos coeficientes de rigidez constitutiva $\boldsymbol{D}$ fique incapaz de modelar a instabilidade por torção em barras comprimidas, além de fornecer resultados discrepantes para 
problemas de flambagem lateral. Conclusões semelhantes também foram alcançadas em [22], linearizando o funcional.

\subsection{Equação constitutiva hiperelástica “completa” de Kirchhoff-St-Venant}

Seja o gradiente das transformações retrorrotacionado $\boldsymbol{F}^{r}$ tal que:

$$
\boldsymbol{F}=\boldsymbol{Q} \boldsymbol{F}^{r}
$$

onde $\boldsymbol{F}^{r}$ é retirado de (2.15), sendo:

$$
\boldsymbol{F}^{r}=\boldsymbol{I}+\gamma^{r} \otimes \boldsymbol{e}_{3}^{r}+\psi_{, \alpha} p \boldsymbol{e}_{3}^{r} \otimes \boldsymbol{e}_{\alpha}^{r}
$$

Sejam $\gamma_{1}^{r}, \gamma_{2}^{r}$ e $\varepsilon$ as três componentes do vetor das deformações $\gamma^{r}$, extraídas de (2.16) e escritas novamente a seguir

$$
\left[\begin{array}{c}
\gamma_{1}^{r} \\
\gamma_{2}^{r} \\
\varepsilon
\end{array}\right]=\left[\begin{array}{c}
\eta_{1}^{r}-x_{2} \kappa_{3}^{r}+p \psi \kappa_{2}^{r} \\
\eta_{2}^{r}+x_{1} \kappa_{3}^{r}-p \psi \kappa_{1}^{r} \\
\eta_{3}^{r}-x_{1} \kappa_{2}^{r}+x_{2} \kappa_{1}^{r}-p^{\prime} \psi
\end{array}\right]=\gamma_{\alpha}^{r} \boldsymbol{e}_{\alpha}^{r}+\varepsilon \boldsymbol{e}_{3}^{r} .
$$

Substituindo (3.9) e (3.10) em (3.2), retendo todos os termos de ordem superior nas deformações e fazendo as manipulações algébricas necessárias, chega-se à forma para o tensor direito de Cauchy: 


$$
\begin{aligned}
\boldsymbol{C}= & \boldsymbol{F}^{T} \boldsymbol{F}=\boldsymbol{F}^{r^{T}} \boldsymbol{F}^{r}=\left(\boldsymbol{I}+\boldsymbol{e}_{3}^{r} \otimes \boldsymbol{\gamma}^{r}+p \psi_{, \alpha} \boldsymbol{e}_{\alpha}^{r} \otimes \boldsymbol{e}_{3}^{r}\right)\left(\boldsymbol{I}+\boldsymbol{\gamma}^{r} \otimes \boldsymbol{e}_{3}^{r}+p \psi_{, \beta} \boldsymbol{e}_{3}^{r} \otimes \boldsymbol{e}_{\beta}^{r}\right) \\
= & \boldsymbol{I}+\boldsymbol{\gamma}^{r} \otimes \boldsymbol{e}_{3}^{r}+p \psi_{, \beta} \boldsymbol{e}_{3}^{r} \otimes \boldsymbol{e}_{\beta}^{r}+\boldsymbol{e}_{3}^{r} \otimes \boldsymbol{\gamma}^{r}+\boldsymbol{e}_{3}^{r} \gamma^{r^{T}} \boldsymbol{\gamma}^{r} \boldsymbol{e}_{3}^{r^{T}}+p \psi_{, \beta} \boldsymbol{e}_{3}^{r} \boldsymbol{\gamma}^{r^{T}} \boldsymbol{e}_{3}^{r} \boldsymbol{e}_{\beta}^{r^{T}}+ \\
& +p \psi_{, \alpha} \boldsymbol{e}_{\alpha}^{r} \otimes \boldsymbol{e}_{3}^{r}+p \psi_{, \alpha} \boldsymbol{e}_{\alpha}^{r} \boldsymbol{e}_{3}^{r^{T}} \boldsymbol{\gamma}^{r} \boldsymbol{e}_{3}^{r^{T}}+p^{2} \psi_{, \alpha} \psi_{, \beta} \boldsymbol{e}_{\alpha}^{r} \boldsymbol{e}_{3}^{r^{T}} \boldsymbol{e}_{3}^{r} \boldsymbol{e}_{\beta}^{r^{T}} \\
= & \boldsymbol{I}+\boldsymbol{\gamma}^{r} \otimes \boldsymbol{e}_{3}^{r}+p \psi_{, \beta} \boldsymbol{e}_{3}^{r} \otimes \boldsymbol{e}_{\beta}^{r}+\boldsymbol{e}_{3}^{r}\left(\boldsymbol{\gamma}^{r} \cdot \boldsymbol{e}_{\beta}^{r}\right) \boldsymbol{e}_{\beta}^{r^{T}}+\boldsymbol{e}_{3}^{r}\left(\boldsymbol{\gamma}^{r} \cdot \boldsymbol{e}_{3}^{r}\right) \boldsymbol{e}_{3}^{r^{T}}+\left(\boldsymbol{\gamma}^{r} \cdot \boldsymbol{\gamma}^{r}\right) \boldsymbol{e}_{3}^{r} \otimes \boldsymbol{e}_{3}^{r}+ \\
& +p \psi_{, \beta}\left(\boldsymbol{\gamma}^{r} \cdot \boldsymbol{e}_{3}^{r}\right) \boldsymbol{e}_{3}^{r} \otimes \boldsymbol{e}_{\beta}^{r}+p \psi_{, \alpha} \boldsymbol{e}_{\alpha}^{r} \otimes \boldsymbol{e}_{3}^{r}+p \psi_{, \alpha}\left(\boldsymbol{\gamma}^{r} \cdot \boldsymbol{e}_{3}^{r}\right) \boldsymbol{e}_{\alpha}^{r} \otimes \boldsymbol{e}_{3}^{r}+p^{2} \psi_{, \alpha} \psi_{, \beta} \boldsymbol{e}_{\alpha}^{r} \otimes \boldsymbol{e}_{\beta}^{r} \\
= & \boldsymbol{I}+\underbrace{\left[p \psi_{, \beta} \boldsymbol{e}_{3}^{r}+\left(\boldsymbol{\gamma}^{r} \cdot \boldsymbol{e}_{\beta}^{r}\right) \boldsymbol{e}_{3}^{r}+p \psi_{, \beta}\left(\boldsymbol{\gamma}^{r} \cdot \boldsymbol{e}_{3}^{r}\right) \boldsymbol{e}_{3}^{r}+p^{2} \psi_{, \alpha} \psi_{, \beta} \boldsymbol{e}_{\alpha}^{r}\right] \otimes \boldsymbol{e}_{\beta}^{r}+}_{\boldsymbol{c}_{\alpha}} \\
& +\underbrace{\left[\boldsymbol{\gamma}^{r}+\left(\boldsymbol{\gamma}^{r} \cdot \boldsymbol{e}_{3}^{r}\right) \boldsymbol{e}_{3}^{r}+\left(\boldsymbol{\gamma}^{r} \cdot \boldsymbol{\gamma}^{r}\right) \boldsymbol{e}_{3}^{r}+p \psi_{, \alpha} \boldsymbol{e}_{\alpha}^{r}+p \psi_{, \alpha}\left(\boldsymbol{\gamma}^{r} \cdot \boldsymbol{e}_{3}^{r}\right) \boldsymbol{e}_{\alpha}^{r}\right] \otimes \boldsymbol{e}_{3}^{r}}_{\boldsymbol{c}_{3}} \\
= & \boldsymbol{I}+\boldsymbol{c}_{\alpha} \otimes \boldsymbol{e}_{\alpha}^{r}+\boldsymbol{c}_{3} \otimes \boldsymbol{e}_{3}^{r} .
\end{aligned}
$$

Assim, o tensor de Green $\boldsymbol{E}$ resulta em:

$$
\boldsymbol{E}=\frac{1}{2}(\boldsymbol{C}-\boldsymbol{I})=\frac{1}{2} \boldsymbol{c}_{\alpha} \otimes \boldsymbol{e}_{\alpha}^{r}+\frac{1}{2} \boldsymbol{c}_{3} \otimes \boldsymbol{e}_{3}^{r}
$$

A lei material de Kirchhoff-Saint-Venant, caracterizando um material hiperelástico, estabeleca que:

$$
\boldsymbol{S}=\lambda(\boldsymbol{I}: \boldsymbol{E}) \boldsymbol{I}+2 \mu \boldsymbol{E}
$$

onde $S$ é o segundo tensor das tensões de Piola-Kirchhoff, e $\lambda$ e $\mu$ fazem o papel das contantes de Lamé (ou módulos de Lamé) para o par $\{\boldsymbol{S}, \boldsymbol{E}\}$.

Utilizando-se a relação conhecida entre $\boldsymbol{S}$ e $\boldsymbol{P}$ dada por

$$
\begin{aligned}
& \boldsymbol{P}=\boldsymbol{F} \boldsymbol{S} \\
& \boldsymbol{P}^{r}=\boldsymbol{F}^{r} \boldsymbol{S},
\end{aligned}
$$

e introduzindo (3.14) e (3.10) nessa última, chega-se a 


$$
\begin{aligned}
\boldsymbol{P}^{r}= & \boldsymbol{F}^{r} \boldsymbol{S}=\left(\boldsymbol{I}+\boldsymbol{\gamma}^{r} \otimes \boldsymbol{e}_{3}^{r}+\psi_{, \alpha} p \boldsymbol{e}_{3}^{r} \otimes \boldsymbol{e}_{\alpha}^{r}\right)(\lambda(\boldsymbol{I}: \boldsymbol{E}) \boldsymbol{I}+2 \mu \boldsymbol{E}) \\
= & \lambda(\boldsymbol{I}: \boldsymbol{E}) \boldsymbol{I}+2 \mu \boldsymbol{E}+\lambda(\boldsymbol{I}: \boldsymbol{E}) \boldsymbol{\gamma}^{r} \otimes \boldsymbol{e}_{3}^{r}+\mu \boldsymbol{\gamma}^{r} \boldsymbol{e}_{3}^{r^{T}} \boldsymbol{c}_{\alpha} \boldsymbol{e}_{\alpha}^{r^{T}}+\mu \boldsymbol{\gamma}^{r} \boldsymbol{e}_{3}^{r^{T}} \boldsymbol{c}_{3} \boldsymbol{e}_{3}^{r^{T}} \\
& +\lambda(\boldsymbol{I}: \boldsymbol{E}) p \psi_{, \alpha} \boldsymbol{e}_{3}^{r} \otimes \boldsymbol{e}_{\alpha}^{r}+\mu p \psi_{, \alpha} \boldsymbol{e}_{3}^{r} \boldsymbol{e}_{\alpha}^{r^{T}} \boldsymbol{c}_{\beta} \boldsymbol{e}_{\beta}^{r^{T}}+\mu p \psi_{, \alpha} \boldsymbol{e}_{3}^{r} \boldsymbol{e}_{\alpha}^{r^{T}} \boldsymbol{c}_{3} \boldsymbol{e}_{3}^{r^{T}} \\
= & \lambda(\boldsymbol{I}: \boldsymbol{E}) \boldsymbol{I}+\mu \boldsymbol{c}_{\alpha} \otimes \boldsymbol{e}_{\alpha}^{r}+\mu \boldsymbol{c}_{3} \otimes \boldsymbol{e}_{3}^{r}+\lambda(\boldsymbol{I}: \boldsymbol{E}) \boldsymbol{\gamma}^{r} \otimes \boldsymbol{e}_{3}^{r}+ \\
& +\mu\left(\boldsymbol{c}_{\alpha} \cdot \boldsymbol{e}_{3}^{r}\right) \boldsymbol{\gamma}^{r} \otimes \boldsymbol{e}_{\alpha}^{r}+\mu\left(\boldsymbol{c}_{3} \cdot \boldsymbol{e}_{3}^{r}\right) \boldsymbol{\gamma}^{r} \otimes \boldsymbol{e}_{3}^{r}+\lambda(\boldsymbol{I}: \boldsymbol{E}) p \psi_{, \alpha} \boldsymbol{e}_{3}^{r} \otimes \boldsymbol{e}_{\alpha}^{r}+ \\
& +\mu p \psi_{, \beta}\left(\boldsymbol{c}_{\alpha} \cdot \boldsymbol{e}_{\beta}^{r}\right) \boldsymbol{e}_{3}^{r} \otimes \boldsymbol{e}_{\alpha}^{r}+\mu p \psi_{, \alpha}\left(\boldsymbol{c}_{3} \cdot \boldsymbol{e}_{\alpha}^{r}\right) \boldsymbol{e}_{3}^{r} \otimes \boldsymbol{e}_{3}^{r} \\
= & \lambda(\boldsymbol{I}: \boldsymbol{E}) \boldsymbol{e}_{i}^{r} \otimes \boldsymbol{e}_{i}^{r}+\left[\mu \boldsymbol{c}_{\alpha}+\mu\left(\boldsymbol{c}_{\alpha} \cdot \boldsymbol{e}_{3}^{r}\right) \boldsymbol{\gamma}^{r}+\lambda(\boldsymbol{I}: \boldsymbol{E}) p \psi_{, \alpha} \boldsymbol{e}_{3}^{r}+\mu p \psi_{, \beta}\left(\boldsymbol{c}_{\alpha} \cdot \boldsymbol{e}_{\beta}^{r}\right) \boldsymbol{e}_{3}^{r}\right] \otimes \boldsymbol{e}_{\alpha}^{r}+ \\
& +\left[\mu \boldsymbol{c}_{3}+\lambda(\boldsymbol{I}: \boldsymbol{E}) \boldsymbol{\gamma}^{r}+\mu\left(\boldsymbol{c}_{3} \cdot \boldsymbol{e}_{3}^{r}\right) \boldsymbol{\gamma}^{r}+\mu p \psi_{, \alpha}\left(\boldsymbol{c}_{3} \cdot \boldsymbol{e}_{\alpha}^{r}\right) \boldsymbol{e}_{3}^{r}\right] \otimes \boldsymbol{e}_{3}^{r} \\
= & \boldsymbol{\tau}_{1}^{r} \otimes \boldsymbol{e}_{1}^{r}+\boldsymbol{\tau}_{2}^{r} \otimes \boldsymbol{e}_{2}^{r}+\boldsymbol{\tau}_{3}^{r} \otimes \boldsymbol{e}_{3}^{r}=\boldsymbol{\tau}_{\alpha}^{r} \otimes \boldsymbol{e}_{\alpha}^{r}+\boldsymbol{\tau}_{3}^{r} \otimes \boldsymbol{e}_{3}^{r} .
\end{aligned}
$$

Desta forma, resulta que os vetores-coluna de $\boldsymbol{P}^{r}$ são dados por:

$$
\begin{aligned}
\boldsymbol{\tau}_{\alpha}^{r} & =\lambda(\boldsymbol{I}: \boldsymbol{E}) \boldsymbol{e}_{\alpha}^{r}+\mu \boldsymbol{c}_{\alpha}+\mu\left(\boldsymbol{c}_{\alpha} \cdot \boldsymbol{e}_{3}^{r}\right) \boldsymbol{\gamma}^{r}+\lambda(\boldsymbol{I}: \boldsymbol{E}) p \psi_{, \alpha} \boldsymbol{e}_{3}^{r}+\mu p \psi_{, \beta}\left(\boldsymbol{c}_{\alpha} \cdot \boldsymbol{e}_{\beta}^{r}\right) \boldsymbol{e}_{3}^{r} \\
\boldsymbol{\tau}_{3}^{r} & =\lambda(\boldsymbol{I}: \boldsymbol{E}) \boldsymbol{e}_{3}^{r}+\mu \boldsymbol{c}_{3}+\lambda(\boldsymbol{I}: \boldsymbol{E}) \boldsymbol{\gamma}^{r}+\mu\left(\boldsymbol{c}_{3} \cdot \boldsymbol{e}_{3}^{r}\right) \boldsymbol{\gamma}^{r}+\mu p \psi_{, \alpha}\left(\boldsymbol{c}_{3} \cdot \boldsymbol{e}_{\alpha}^{r}\right) \boldsymbol{e}_{3}^{r} .
\end{aligned}
$$

Uma forma alternativa de se escrever essas expressões é através das expansões nas direções $\boldsymbol{e}_{\alpha}^{r}$ e $\boldsymbol{e}_{3}^{r}$,ou seja:

$$
\begin{aligned}
\boldsymbol{\tau}_{\alpha}^{r}= & {\left[\lambda(\boldsymbol{I}: \boldsymbol{E})+\mu p^{2} \psi_{, \alpha}^{2}+\mu\left(\boldsymbol{c}_{\alpha} \cdot \boldsymbol{e}_{3}^{r}\right)\left(\boldsymbol{\gamma}^{r} \cdot \boldsymbol{e}_{\alpha}^{r}\right)\right] \boldsymbol{e}_{\alpha}^{r}+\left[\mu p \psi_{, \alpha}+\mu\left(\boldsymbol{\gamma}^{r} \cdot \boldsymbol{e}_{\alpha}^{r}\right)\right.} \\
& \left.+\mu p \psi_{, \alpha}\left(\boldsymbol{\gamma}^{r} \cdot \boldsymbol{e}_{3}^{r}\right)+\mu\left(\boldsymbol{c}_{\alpha} \cdot \boldsymbol{e}_{3}^{r}\right)\left(\boldsymbol{\gamma}^{r} \cdot \boldsymbol{e}_{3}^{r}\right)+\lambda(\boldsymbol{I}: \boldsymbol{E}) p \psi_{, \alpha}+\mu p \psi_{, \beta}\left(\boldsymbol{c}_{\alpha} \cdot \boldsymbol{e}_{\beta}^{r}\right)\right] \boldsymbol{e}_{3}^{r} \\
\boldsymbol{\tau}_{3}^{r}= & {\left[\mu\left(\boldsymbol{\gamma}^{r} \cdot \boldsymbol{e}_{\alpha}^{r}\right)+\mu p \psi_{, \alpha}+\mu p \psi_{, \alpha}\left(\boldsymbol{\gamma}^{r} \cdot \boldsymbol{e}_{3}^{r}\right)+\lambda(\boldsymbol{I}: \boldsymbol{E})\left(\boldsymbol{\gamma}^{r} \cdot \boldsymbol{e}_{\alpha}^{r}\right)+\mu\left(\boldsymbol{c}_{3} \cdot \boldsymbol{e}_{3}^{r}\right)\left(\boldsymbol{\gamma}^{r} \cdot \boldsymbol{e}_{\alpha}^{r}\right)\right] \boldsymbol{e}_{\alpha}^{r} } \\
& +\left[\lambda(\boldsymbol{I}: \boldsymbol{E})+\mu\left(\boldsymbol{\gamma}^{r} \cdot \boldsymbol{e}_{3}^{r}\right)+\mu\left(\boldsymbol{\gamma}^{r} \cdot \boldsymbol{e}_{3}^{r}\right)+\mu\left(\boldsymbol{\gamma}^{r} \cdot \boldsymbol{\gamma}^{r}\right)+\lambda(\boldsymbol{I}: \boldsymbol{E})\left(\boldsymbol{\gamma}^{r} \cdot \boldsymbol{e}_{3}^{r}\right)+\right. \\
& \left.+\mu\left(\boldsymbol{c}_{\alpha} \cdot \boldsymbol{e}_{3}^{r}\right)\left(\boldsymbol{\gamma}^{r} \cdot \boldsymbol{e}_{3}^{r}\right)+\mu p \psi_{, \alpha}\left(\boldsymbol{c}_{3} \cdot \boldsymbol{e}_{\alpha}^{r}\right)\right] \boldsymbol{e}_{3}^{r} .
\end{aligned}
$$

Como, de (3.13), tem-se

$$
\begin{aligned}
\boldsymbol{I}: \boldsymbol{E} & =\operatorname{tr}(\boldsymbol{E})=\frac{1}{2}\left(\boldsymbol{c}_{1} \cdot \boldsymbol{e}_{1}^{r}+\boldsymbol{c}_{2} \cdot \boldsymbol{e}_{2}^{r}+\boldsymbol{c}_{3} \cdot \boldsymbol{e}_{3}^{r}\right)= \\
& =\frac{1}{2}\left[p^{2} \psi_{, 1}^{2}+p^{2} \psi_{, 2}^{2}+\left(\boldsymbol{\gamma}^{r} \cdot \boldsymbol{e}_{3}^{r}\right)+\left(\boldsymbol{\gamma}^{r} \cdot \boldsymbol{e}_{3}^{r}\right)+\left(\boldsymbol{\gamma}^{r} \cdot \boldsymbol{\gamma}^{r}\right)\right] \\
& =\left(\boldsymbol{\gamma}^{r} \cdot \boldsymbol{e}_{3}^{r}\right)+\frac{1}{2}\left(\boldsymbol{\gamma}^{r} \cdot \boldsymbol{\gamma}^{r}\right)+\frac{1}{2} p^{2} \psi_{, \beta}^{2} \\
& =\varepsilon+\frac{1}{2} \varepsilon^{2}+\frac{1}{2} \boldsymbol{\gamma}_{\beta}^{r^{2}}+\frac{1}{2} p^{2} \psi_{, \beta}^{2},
\end{aligned}
$$


pode-se também escrever para $\tau_{3}^{r}$ :

$$
\begin{aligned}
\boldsymbol{\tau}_{3 \alpha}^{r}= & \mu \gamma_{\alpha}^{r}+\mu p \psi_{, \alpha}+\mu p \psi_{, \alpha} \varepsilon+\lambda\left(\varepsilon+\frac{1}{2} \varepsilon^{2}+\frac{1}{2} \gamma_{\beta}^{r^{2}}+\frac{1}{2} p^{2} \psi_{, \beta}^{2}\right) \gamma_{\alpha}^{r} \\
& +\mu\left(\varepsilon+\varepsilon+\gamma_{\beta}^{r^{2}}+\varepsilon^{2}\right) \boldsymbol{\gamma}_{\alpha}^{r} \\
= & \mu \gamma_{\alpha}^{r}+\mu p \psi_{, \alpha}+\mu p \psi_{, \alpha} \varepsilon+\lambda \varepsilon \gamma_{\alpha}^{r}+\frac{1}{2} \lambda \varepsilon^{2} \gamma_{\alpha}^{r}+\frac{1}{2} \lambda \boldsymbol{\gamma}_{\beta}^{r^{2}} \gamma_{\alpha}^{r}+ \\
& +\frac{1}{2} \lambda p^{2} \psi_{, \beta}^{2} \gamma_{\alpha}^{r}+2 \mu \varepsilon \gamma_{\alpha}^{r}+\mu \gamma_{\beta}^{r^{2}} \gamma_{\alpha}^{r}+\mu \varepsilon^{2} \gamma_{\alpha}^{r} \\
\boldsymbol{\tau}_{33}^{r}= & \lambda \varepsilon+\frac{1}{2} \lambda \varepsilon^{2}+\frac{1}{2} \lambda \gamma_{\beta}^{r^{2}}+\frac{1}{2} \lambda p^{2} \psi_{, \beta}^{2}+2 \mu \varepsilon+\mu \boldsymbol{\gamma}_{\beta}^{r^{2}}+\mu \varepsilon^{2}+\lambda \varepsilon^{2}+ \\
& +\frac{1}{2} \lambda \varepsilon^{3}+\frac{1}{2} \lambda \gamma_{\beta}^{r^{2}} \varepsilon+\frac{1}{2} \lambda p^{2} \psi_{, \beta}^{2} \varepsilon+\mu \varepsilon^{2}+\mu \varepsilon^{2}+\mu \gamma_{\beta}^{r^{2}} \varepsilon+ \\
& +\mu \varepsilon^{3}+\mu p \psi_{, \alpha} \gamma_{\alpha}^{r}+\mu p^{2} \psi_{, \alpha}^{2}+\mu p^{2} \psi_{, \alpha}^{2} \varepsilon
\end{aligned}
$$

Rearrumando a expressão acima, de forma a que se tenha os termos cúbicos por último, temse:

$$
\begin{aligned}
\boldsymbol{\tau}_{3 \alpha}^{r}= & \mu \boldsymbol{\gamma}_{\alpha}^{r}+\mu p \psi_{, \alpha}+\mu p \psi_{, \alpha} \varepsilon+(\lambda+2 \mu) \varepsilon \boldsymbol{\gamma}_{\alpha}^{r}+\frac{1}{2}(\lambda+2 \mu) \varepsilon^{2} \boldsymbol{\gamma}_{\alpha}^{r}+ \\
& +\frac{1}{2}(\lambda+2 \mu) \boldsymbol{\gamma}_{\beta}^{r^{2}} \boldsymbol{\gamma}_{\alpha}^{r}+\frac{1}{2} \lambda p^{2} \psi_{, \beta}^{2} \gamma_{\alpha}^{r} \\
\boldsymbol{\tau}_{33}^{r}= & (\lambda+2 \mu) \varepsilon+\frac{3}{2}(\lambda+2 \mu) \varepsilon^{2}+\frac{1}{2}(\lambda+2 \mu) \boldsymbol{\gamma}_{\beta}^{r^{2}}+\frac{1}{2}(\lambda+2 \mu) p^{2} \psi_{, \beta}^{2}+ \\
& +\frac{1}{2}(\lambda+2 \mu) \varepsilon^{3}+\frac{1}{2}(\lambda+2 \mu) \boldsymbol{\gamma}_{\beta}^{r^{2}} \varepsilon+\frac{1}{2}(\lambda+2 \mu) p^{2} \psi_{, \beta}^{2} \varepsilon
\end{aligned}
$$

Para $\boldsymbol{\tau}_{1}^{r}$, analogamente, tem-se:

$$
\begin{aligned}
\boldsymbol{\tau}_{1}^{r}= & \lambda(\boldsymbol{I}: \boldsymbol{E}) \boldsymbol{e}_{1}^{r}+\mu \boldsymbol{c}_{1}+\mu\left(\boldsymbol{c}_{1} \cdot \boldsymbol{e}_{3}^{r}\right) \boldsymbol{\gamma}^{r}+\lambda(\boldsymbol{I}: \boldsymbol{E}) p \psi_{, 1} \boldsymbol{e}_{3}^{r}+ \\
& +\mu p \psi_{, 1}\left(\boldsymbol{c}_{1} \cdot \boldsymbol{e}_{1}^{r}\right) \boldsymbol{e}_{3}^{r}+\mu p \psi_{, 2}\left(\boldsymbol{c}_{1} \cdot \boldsymbol{e}_{2}^{r}\right) \boldsymbol{e}_{3}^{r} \\
\boldsymbol{\tau}_{1_{1}}^{r}= & \lambda\left(\varepsilon+\frac{1}{2} \varepsilon^{2}+\frac{1}{2} \boldsymbol{\gamma}_{\beta}^{r^{2}}+\frac{1}{2} p^{2} \psi_{, \beta}^{2}\right)+\mu p^{2} \psi_{, 1}^{2}+\mu\left(p \psi_{, 1}+\boldsymbol{\gamma}_{1}^{r}+p \psi_{, 1} \varepsilon\right) \boldsymbol{\gamma}_{1}^{r} \\
\boldsymbol{\tau}_{1_{2}}^{r}= & \mu\left(p^{2} \psi_{, 1} \psi_{, 2}\right)+\mu\left(p \psi_{, 1}+\gamma_{1}^{r}+p \psi_{, 1} \varepsilon\right) \boldsymbol{\gamma}_{2}^{r} \\
\boldsymbol{\tau}_{1_{3}}^{r}= & \mu\left(p \psi_{, 1}+\gamma_{1}^{r}+p \psi_{, 1} \varepsilon\right)+\mu\left(p \psi_{, 1}+\gamma_{1}^{r}+p \psi_{, 1} \varepsilon\right) \varepsilon+\lambda p \psi_{, 1}\left(\varepsilon+\frac{1}{2} \varepsilon^{2}+\frac{1}{2} \boldsymbol{\gamma}_{\beta}^{r^{2}}+\frac{1}{2} p^{2} \psi_{, \beta}^{2}\right)+ \\
& +\mu p \psi_{, 1}\left(p^{2} \psi_{, 1}^{2}\right)+\mu p \psi_{, 2}\left(p^{2} \psi_{, 1} \psi_{, 2}\right) .
\end{aligned}
$$


E para $\tau_{2}^{r}$, tem-se:

$$
\begin{aligned}
\boldsymbol{\tau}_{2}^{r}= & \lambda(\boldsymbol{I}: \boldsymbol{E}) \boldsymbol{e}_{2}^{r}+\mu \boldsymbol{c}_{2}+\mu\left(\boldsymbol{c}_{2} \cdot \boldsymbol{e}_{3}^{r}\right) \boldsymbol{\gamma}^{r}+\lambda(\boldsymbol{I}: \boldsymbol{E}) p \psi_{, 2} \boldsymbol{e}_{3}^{r}+ \\
& +\mu p \psi_{, 1}\left(\boldsymbol{c}_{2} \cdot \boldsymbol{e}_{1}^{r}\right) \boldsymbol{e}_{3}^{r}+\mu p \psi_{, 2}\left(\boldsymbol{c}_{2} \cdot \boldsymbol{e}_{2}^{r}\right) \boldsymbol{e}_{3}^{r} \\
\boldsymbol{\tau}_{2_{1}}^{r}= & \mu\left(p^{2} \psi_{, 1} \psi_{, 2}\right)+\mu\left(p \psi_{, 2}+\boldsymbol{\gamma}_{2}^{r}+p \psi_{, 2} \varepsilon\right) \boldsymbol{\gamma}_{1}^{r} \\
\boldsymbol{\tau}_{2_{2}}^{r}= & \lambda\left(\varepsilon+\frac{1}{2} \varepsilon^{2}+\frac{1}{2} \boldsymbol{\gamma}_{\beta}^{r^{2}}+\frac{1}{2} p^{2} \psi_{, \beta}^{2}\right)+\mu\left(p^{2} \psi_{, 2}^{2}\right)+\mu\left(p \psi_{, 2}+\boldsymbol{\gamma}_{2}^{r}+p \psi_{, 2} \varepsilon\right) \boldsymbol{\gamma}_{2}^{r} \\
\boldsymbol{\tau}_{2_{3}}^{r}= & \mu\left(p \psi_{, 2}+\boldsymbol{\gamma}_{2}^{r}+p \psi_{, 2} \varepsilon\right)+\mu\left(p \psi_{, 2}+\boldsymbol{\gamma}_{2}^{r}+p \psi_{, 2} \varepsilon\right) \varepsilon+\lambda p \psi_{, 2}\left(\varepsilon+\frac{1}{2} \varepsilon^{2}+\frac{1}{2} \boldsymbol{\gamma}_{\beta}^{r^{2}}+\frac{1}{2} p^{2} \psi_{, \beta}^{2}\right)+ \\
& +\mu p \psi_{, 1}\left(p^{2} \psi_{, 2}^{2}\right)+\mu p \psi_{, 1}\left(p^{2} \psi_{, 1} \psi_{, 2}\right) .
\end{aligned}
$$

A matriz dos módulos elásticos de rigidez tangente $\boldsymbol{D}$, que decorre dessas tensões, pode ser obtida como explicado a seguir. Por definição, $\boldsymbol{D}$ é dado por (2.60), isto é,

$$
\boldsymbol{D}=\frac{\partial \boldsymbol{\sigma}^{r}}{\partial \boldsymbol{\varepsilon}^{r}}=\left[\begin{array}{llll}
\frac{\partial \boldsymbol{n}^{r}}{\partial \boldsymbol{\eta}^{r}} & \frac{\partial \boldsymbol{n}^{r}}{\partial \boldsymbol{k}^{r}} & \frac{\partial \boldsymbol{n}^{r}}{\partial p} & \frac{\partial \boldsymbol{n}^{r}}{\partial p^{\prime}} \\
\frac{\partial \boldsymbol{m}^{r}}{\partial \boldsymbol{\eta}^{r}} & \frac{\partial m^{r}}{\partial \kappa^{r}} & \frac{\partial m^{r}}{\partial p} & \frac{\partial m^{r}}{\partial p^{\prime}} \\
\frac{\partial Q}{\partial \boldsymbol{\eta}^{r}} & \frac{\partial Q}{\partial \boldsymbol{\kappa}^{r}} & \frac{\partial Q}{\partial p} & \frac{\partial Q}{\partial p^{\prime}} \\
\frac{\partial B}{\partial \boldsymbol{\eta}^{r}} & \frac{\partial B}{\partial \boldsymbol{k}^{r}} & \frac{\partial B}{\partial p} & \frac{\partial B}{\partial p^{\prime}}
\end{array}\right]=\left[\begin{array}{llll}
\boldsymbol{D}_{\eta \eta} & \boldsymbol{D}_{\eta \kappa} & \boldsymbol{D}_{\eta p} & \boldsymbol{D}_{\eta p^{\prime}} \\
& \boldsymbol{D}_{k k} & \boldsymbol{D}_{k p} & \boldsymbol{D}_{k p^{\prime}} \\
& \boldsymbol{D}_{p p} & \boldsymbol{D}_{p p^{\prime}} \\
& & \boldsymbol{D}_{p^{\prime} p^{\prime}}
\end{array}\right]
$$

Para o cálculo das derivadas envolvidas nas submatrizes de $\boldsymbol{D}$, torna-se necessário as seguintes grandezas auxiliares: 


$$
\begin{aligned}
& \frac{\partial(\boldsymbol{I}: \boldsymbol{E})}{\partial \boldsymbol{\gamma}^{r}}=\boldsymbol{e}_{3}^{r^{T}}+\boldsymbol{\gamma}^{r^{T}}=\left(\boldsymbol{e}_{3}^{r}+\boldsymbol{\gamma}^{r}\right)^{T} \\
& \frac{\partial \boldsymbol{c}_{3}}{\partial \boldsymbol{\gamma}^{r}}=\boldsymbol{I}+\boldsymbol{e}_{3}^{r} \otimes \boldsymbol{e}_{3}^{r}+2 \boldsymbol{e}_{3}^{r} \otimes \boldsymbol{\gamma}^{r}+p \psi_{, \alpha} \boldsymbol{e}_{\alpha}^{r} \otimes \boldsymbol{e}_{3}^{r} \\
& \begin{aligned}
& \frac{\boldsymbol{d}(\boldsymbol{I}: \boldsymbol{E}) \boldsymbol{\gamma}^{r}}{\partial \boldsymbol{\gamma}^{r}}=\boldsymbol{\gamma}^{r} \frac{\partial(\boldsymbol{I}: \boldsymbol{E})}{\partial \boldsymbol{\gamma}^{r}}+(\boldsymbol{I}: \boldsymbol{E}) \frac{\partial \boldsymbol{\gamma}^{r}}{\partial \boldsymbol{\gamma}^{r}}=\boldsymbol{\gamma}^{r} \otimes\left(\boldsymbol{e}_{3}^{r}+\boldsymbol{\gamma}^{r}\right)+(\boldsymbol{I}: \boldsymbol{E}) \boldsymbol{I} \\
& \frac{\partial\left(\boldsymbol{c}_{3} \cdot \boldsymbol{e}_{3}^{r}\right) \boldsymbol{\gamma}^{r}}{\partial \boldsymbol{\gamma}^{r}}=\boldsymbol{\gamma}^{r} \frac{\partial\left(\boldsymbol{c}_{3} \cdot \boldsymbol{e}_{3}^{r}\right)}{\partial \boldsymbol{\gamma}^{r}}+\left(\boldsymbol{c}_{3} \cdot \boldsymbol{e}_{3}^{r}\right) \frac{\partial \boldsymbol{\gamma}^{r}}{\partial \boldsymbol{\gamma}^{r}}=\boldsymbol{\gamma}^{r} \otimes\left(\boldsymbol{e}_{3}^{r}+\boldsymbol{e}_{3}^{r}+2 \boldsymbol{\gamma}^{r}\right)+\left(\boldsymbol{c}_{3} \cdot \boldsymbol{e}_{3}^{r}\right) \boldsymbol{I} \\
& \frac{\partial\left(\boldsymbol{c}_{3} \cdot \boldsymbol{e}_{\alpha}^{r}\right) \boldsymbol{e}_{3}^{r}}{\partial \boldsymbol{\gamma}^{r}}=\boldsymbol{e}_{3}^{r} \otimes \boldsymbol{e}_{\alpha}^{r}+p \psi_{, \alpha} \boldsymbol{e}_{3}^{r} \otimes \boldsymbol{e}_{3}^{r} \\
& \frac{\partial(\boldsymbol{I}: \boldsymbol{E})}{\partial p}=\frac{\partial}{\partial p}\left(\boldsymbol{\gamma}^{r} \cdot \boldsymbol{e}_{3}^{r}+\frac{1}{2} \boldsymbol{\gamma}^{r} \cdot \boldsymbol{\gamma}^{r}+\frac{1}{2} p^{2} \psi_{, \beta}^{2}\right) \\
& \quad=\boldsymbol{\gamma}^{r} \cdot \psi \boldsymbol{\kappa}^{r} \times \boldsymbol{e}_{3}^{r}+p \psi_{, \beta}^{2} \\
&=\boldsymbol{\gamma}_{1}^{r}\left(\psi \boldsymbol{\kappa}_{2}^{r}\right)+\boldsymbol{\gamma}_{2}^{r}\left(-\psi \boldsymbol{\kappa}_{1}^{r}\right)+p \psi_{, 1}^{2}+p \psi_{, 2}^{2}
\end{aligned}
\end{aligned}
$$

$$
\begin{aligned}
\frac{\partial \boldsymbol{c}_{3}}{\partial p}= & \psi \boldsymbol{\kappa}^{r} \times \boldsymbol{e}_{3}^{r}+\boldsymbol{e}_{3}^{r} \cdot \psi \boldsymbol{\kappa}^{r} \times \boldsymbol{e}_{3}^{r}+2 \psi\left(\boldsymbol{\gamma}^{r} \cdot \boldsymbol{\kappa}^{r} \times \boldsymbol{e}_{3}^{r}\right) \boldsymbol{e}_{3}^{r}+\psi_{, \alpha} \boldsymbol{e}_{\alpha}^{r}+ \\
& +\psi_{, \alpha}\left(\boldsymbol{\gamma}^{r} \cdot \boldsymbol{e}_{3}^{r}\right) \boldsymbol{e}_{\alpha}^{r}+p \psi_{, \alpha}(\circ) \boldsymbol{e}_{\alpha}^{r} \\
= & \psi \boldsymbol{\kappa}^{r} \times \boldsymbol{e}_{3}^{r}+2 \psi\left(\boldsymbol{\gamma}^{r} \cdot \boldsymbol{\kappa}^{r} \times \boldsymbol{e}_{3}^{r}\right) \boldsymbol{e}_{3}^{r}+\psi_{, \alpha} \boldsymbol{e}_{\alpha}^{r}+\psi_{, \alpha}\left(\gamma^{r} \cdot \boldsymbol{e}_{3}^{r}\right) \boldsymbol{e}_{\alpha}^{r}
\end{aligned}
$$$$
\frac{\partial(\boldsymbol{I}: \boldsymbol{E}) \boldsymbol{\gamma}^{r}}{\partial p}=\frac{\partial(\boldsymbol{I}: \boldsymbol{E})}{\partial p} \boldsymbol{\gamma}^{r}+(\boldsymbol{I}: \boldsymbol{E}) \frac{\partial \boldsymbol{\gamma}^{r}}{\partial p}
$$$$
=\left(\boldsymbol{\gamma}^{r} \cdot \psi \boldsymbol{\kappa}^{r} \times \boldsymbol{e}_{3}^{r}+p \psi_{, \beta}^{2}\right) \boldsymbol{\gamma}^{r}+(\boldsymbol{I}: \boldsymbol{E}) \psi \boldsymbol{\kappa}^{r} \times \boldsymbol{e}_{3}^{r}
$$$$
\frac{\partial\left(\boldsymbol{c}_{3} \cdot \boldsymbol{e}_{3}^{r}\right) \boldsymbol{\gamma}^{r}}{\partial p}=\frac{\partial\left(\boldsymbol{c}_{3} \cdot \boldsymbol{e}_{3}^{r}\right)}{\partial p} \boldsymbol{\gamma}^{r}+\left(\boldsymbol{c}_{3} \cdot \boldsymbol{e}_{3}^{r}\right) \frac{\partial \boldsymbol{\gamma}^{r}}{\partial p}=
$$$$
=\left(o+o+2 \psi \boldsymbol{\gamma}^{r} \cdot \boldsymbol{\kappa}^{r} \times \boldsymbol{e}_{3}^{r}\right) \boldsymbol{\gamma}^{r}+\left(\boldsymbol{c}_{3} \cdot \boldsymbol{e}_{3}^{r}\right) \psi \boldsymbol{\kappa}^{r} \times \boldsymbol{e}_{3}^{r}
$$ 


$$
\begin{aligned}
& \frac{\partial}{\partial p}\left(p \psi_{, \alpha}\left(\boldsymbol{c}_{3} \cdot \boldsymbol{e}_{3}^{r}\right) \boldsymbol{e}_{3}^{r}\right)=\psi_{, \alpha}\left(\boldsymbol{c}_{3} \cdot \boldsymbol{e}_{3}^{r}\right) \boldsymbol{e}_{3}^{r}+p \psi_{, \alpha} \frac{\partial}{\partial p}\left(\boldsymbol{e}_{\alpha}^{r^{T}} \boldsymbol{c}_{3}\right) \boldsymbol{e}_{3}^{r} \\
& =\psi_{, \alpha}\left(\boldsymbol{c}_{3} \cdot \boldsymbol{e}_{3}^{r}\right) \boldsymbol{e}_{3}^{r}+p \psi_{, \alpha}\left(\boldsymbol { e } _ { \alpha } ^ { r } \cdot \left(\psi \boldsymbol{\kappa}^{r} \times \boldsymbol{e}_{3}^{r}+2 \psi\left(\boldsymbol{\gamma}^{r} \cdot \boldsymbol{\kappa}^{r} \times \boldsymbol{e}_{3}^{r}\right) \boldsymbol{e}_{3}^{r}+\right.\right. \\
& \left.+\psi_{, \alpha} \boldsymbol{e}_{\alpha}^{r}+\psi_{, \alpha}\left(\boldsymbol{\gamma}^{r} \cdot \boldsymbol{e}_{3}^{r}\right) \boldsymbol{e}_{\alpha}^{r}\right) \boldsymbol{e}_{3}^{r} \\
& \frac{\partial(\boldsymbol{I}: \boldsymbol{E})}{\partial p^{\prime}}=\frac{\partial}{\partial p^{\prime}}\left(\boldsymbol{\gamma}^{r} \cdot \boldsymbol{e}_{3}^{r}+\frac{1}{2} \boldsymbol{\gamma}^{r} \cdot \boldsymbol{\gamma}^{r}+\frac{1}{2} p^{2} \psi_{, \beta}^{2}\right)= \\
& =\psi+\psi \gamma^{r} \cdot \boldsymbol{e}_{3}^{r} \\
& \frac{\partial \boldsymbol{c}_{3}}{\partial p^{\prime}}=\psi \boldsymbol{e}_{3}^{r}+\psi \boldsymbol{e}_{3}^{r}+2\left(\boldsymbol{\gamma}^{r} \cdot \psi \boldsymbol{e}_{3}^{r}\right) \boldsymbol{e}_{3}^{r}+p \psi_{, \alpha} \psi \boldsymbol{e}_{\alpha}^{r} \\
& =2 \psi \boldsymbol{e}_{3}^{r}+2 \psi\left(\boldsymbol{\gamma}^{r} \cdot \boldsymbol{e}_{3}^{r}\right) \boldsymbol{e}_{3}^{r}+p \psi_{, \alpha} \psi \boldsymbol{e}_{\alpha}^{r} \\
& \frac{\partial(\boldsymbol{I}: \boldsymbol{E}) \boldsymbol{\gamma}^{r}}{\partial p^{\prime}}=\frac{\partial(\boldsymbol{I}: \boldsymbol{E})}{\partial p^{\prime}} \boldsymbol{\gamma}^{r}+(\boldsymbol{I}: \boldsymbol{E}) \frac{\partial \boldsymbol{\gamma}^{r}}{\partial p^{\prime}}= \\
& =\left(\psi+\psi \boldsymbol{\gamma}^{r} \cdot \boldsymbol{e}_{3}^{r}\right) \boldsymbol{\gamma}^{r}+(\boldsymbol{I}: \boldsymbol{E}) \psi \boldsymbol{e}_{3}^{r} \\
& \frac{\partial\left(\boldsymbol{c}_{3} \cdot \boldsymbol{e}_{3}^{r}\right) \boldsymbol{\gamma}^{r}}{\partial p^{\prime}}=\frac{\partial\left(\boldsymbol{c}_{3} \cdot \boldsymbol{e}_{3}^{r}\right)}{\partial p^{\prime}} \boldsymbol{\gamma}^{r}+\left(\boldsymbol{c}_{3} \cdot \boldsymbol{e}_{3}^{r}\right) \frac{\partial \boldsymbol{\gamma}^{r}}{\partial p^{\prime}}= \\
& =(\underbrace{\boldsymbol{e}_{3}^{r} \cdot \psi \boldsymbol{e}_{3}^{r}}_{\psi}+\boldsymbol{e}_{3}^{r} \cdot \psi \boldsymbol{e}_{3}^{r}+2 \psi \boldsymbol{\gamma}^{r} \cdot \boldsymbol{e}_{3}^{r}) \boldsymbol{\gamma}^{r}+\left(\boldsymbol{c}_{3} \cdot \boldsymbol{e}_{3}^{r}\right) \psi \boldsymbol{e}_{3}^{r} \\
& \frac{\partial\left(\boldsymbol{c}_{3} \cdot \boldsymbol{e}_{3}^{r}\right) \boldsymbol{e}_{3}^{r}}{\partial p^{\prime}}=\left(\boldsymbol{e}_{\alpha}^{r^{T}} \frac{\partial \boldsymbol{c}_{3}}{\partial p^{\prime}}\right) \boldsymbol{e}_{3}^{r} \\
& =\left[\boldsymbol{e}_{\alpha}^{r}\left(2 \psi \boldsymbol{e}_{3}^{r}+2 \psi\left(\boldsymbol{\gamma}^{r} \cdot \boldsymbol{e}_{3}^{r}\right) \boldsymbol{e}_{3}^{r}+p \psi_{, \alpha} \psi \boldsymbol{e}_{\alpha}^{r}\right)\right] \boldsymbol{e}_{3}^{r} \\
& =p \psi_{, \alpha} \psi \boldsymbol{e}_{3}^{r}
\end{aligned}
$$

Com isso, pode-se escrever: 


$$
\begin{aligned}
& \frac{\partial \boldsymbol{\tau}_{3}^{r}}{\partial \boldsymbol{\gamma}^{r}}=\boldsymbol{C}_{3}=\lambda \boldsymbol{e}_{3}^{r} \otimes\left(\boldsymbol{e}_{3}^{r}+\boldsymbol{\gamma}^{r}\right)+\mu\left(\boldsymbol{I}+\boldsymbol{e}_{3}^{r} \otimes \boldsymbol{e}_{3}^{r}+2 \boldsymbol{e}_{3}^{r} \otimes \boldsymbol{\gamma}^{r}+p \psi_{, \alpha} \boldsymbol{e}_{\alpha}^{r} \otimes \boldsymbol{e}_{3}^{r}\right)+ \\
& +\lambda\left[\boldsymbol{\gamma}^{r} \otimes\left(\boldsymbol{e}_{3}^{r}+\boldsymbol{\gamma}^{r}\right)+(\boldsymbol{I}: \boldsymbol{E}) \boldsymbol{I}\right]+ \\
& +\mu\left[\boldsymbol{\gamma}^{r} \otimes\left(2 \boldsymbol{e}_{3}^{r}+2 \boldsymbol{\gamma}^{r}\right)+\left(\boldsymbol{c}_{3} \cdot \boldsymbol{e}_{3}^{r}\right) \boldsymbol{I}\right]+ \\
& +\mu p \psi_{, \alpha}\left(\boldsymbol{e}_{3}^{r} \otimes \boldsymbol{e}_{\alpha}^{r}+p \psi_{, \alpha} \boldsymbol{e}_{3}^{r} \otimes \boldsymbol{e}_{3}^{r}\right) \\
& \frac{\partial \boldsymbol{\tau}_{3}^{r}}{\partial p}=\boldsymbol{c}=\lambda\left[\boldsymbol{\gamma}^{r} \cdot\left(\psi \boldsymbol{\kappa}^{r} \times \boldsymbol{e}_{3}^{r}\right)+p \psi_{, \beta}^{2}\right] \boldsymbol{e}_{3}^{r}+ \\
& +\mu\left[\psi \boldsymbol{\kappa}^{r} \times \boldsymbol{e}_{3}^{r}+2 \psi\left(\boldsymbol{\gamma}^{r}\left(\boldsymbol{\kappa}^{r} \times \boldsymbol{e}_{3}^{r}\right)\right) \boldsymbol{e}_{3}^{r}+\psi_{, \alpha} \boldsymbol{e}_{\alpha}^{r}+\psi_{, \alpha}\left(\boldsymbol{\gamma}^{r} \cdot \boldsymbol{e}_{3}^{r}\right) \boldsymbol{e}_{\alpha}^{r}\right] \\
& +\lambda\left[\left(\boldsymbol{\gamma}^{r} \cdot \psi \boldsymbol{\kappa}^{r} \times \boldsymbol{e}_{3}^{r}+p \psi_{, \beta}^{2}\right) \boldsymbol{\gamma}^{r}+(\boldsymbol{I}: \boldsymbol{E}) \psi \boldsymbol{\kappa}^{r} \times \boldsymbol{e}_{3}^{r}\right]+ \\
& +\mu\left[\left(2 \psi \boldsymbol{\gamma}^{r} \cdot \boldsymbol{\kappa}^{r} \times \boldsymbol{e}_{3}^{r}\right) \boldsymbol{\gamma}^{r}+\left(\boldsymbol{c}_{3} \cdot \boldsymbol{e}_{3}^{r}\right) \psi \boldsymbol{\kappa}^{r} \times \boldsymbol{e}_{3}^{r}\right]+ \\
& +\mu\left[\psi_{, \alpha}\left(\boldsymbol{c}_{3} \cdot \boldsymbol{e}_{3}^{r}\right) \boldsymbol{e}_{3}^{r}+p \psi_{, \alpha}\left(\boldsymbol { e } _ { \alpha } ^ { r } \cdot \left(\psi \boldsymbol{\kappa}^{r} \times \boldsymbol{e}_{3}^{r}+2 \psi\left(\boldsymbol{\gamma}^{r} \cdot \boldsymbol{\kappa}^{r} \times \boldsymbol{e}_{3}^{r}\right) \boldsymbol{e}_{3}^{r}+\right.\right.\right. \\
& \left.\left.+\psi_{, \alpha} \boldsymbol{e}_{\alpha}^{r}+\psi_{, \alpha}\left(\boldsymbol{\gamma}^{r} \cdot \boldsymbol{e}_{3}^{r}\right) \boldsymbol{e}_{\alpha}^{r}\right) \boldsymbol{e}_{3}^{r}\right] \\
& \frac{\partial \boldsymbol{\tau}_{3}^{r}}{\partial p^{\prime}}=\boldsymbol{d}_{3}=\lambda\left(\psi+\psi \boldsymbol{\gamma}^{r} \cdot \boldsymbol{e}_{3}^{r}\right) \boldsymbol{e}_{3}^{r}+\mu\left[2 \psi \boldsymbol{e}_{3}^{r}+2 \psi\left(\boldsymbol{\gamma}^{r} \cdot \boldsymbol{e}_{3}^{r}\right) \boldsymbol{e}_{3}^{r}+p \psi_{, \alpha} \psi \boldsymbol{e}_{\alpha}^{r}\right]+ \\
& +\lambda\left[\left(\psi+\psi \boldsymbol{\gamma}^{r} \cdot \boldsymbol{e}_{3}^{r}\right) \boldsymbol{\gamma}^{r}+(\boldsymbol{I}: \boldsymbol{E}) \psi \boldsymbol{e}_{3}^{r}\right]+ \\
& +\mu\left[\left(2 \psi+2 \psi \boldsymbol{\gamma}^{r} \cdot \boldsymbol{e}_{3}^{r}\right) \boldsymbol{\gamma}^{r}+\left(\boldsymbol{c}_{3} \cdot \boldsymbol{e}_{3}^{r}\right) \psi \boldsymbol{e}_{3}^{r}\right]+\mu p \psi_{, \alpha}\left(p \psi_{, \alpha} \psi \boldsymbol{e}_{3}^{r}\right) \\
& \frac{\partial\left(\boldsymbol{\tau}_{\alpha}^{r} \cdot \boldsymbol{e}_{3}^{r}\right)}{\partial p}=\mu[\psi_{, \alpha}+\overbrace{\boldsymbol{e}_{\alpha}^{r} \cdot\left(\psi \boldsymbol{\kappa}^{r} \times \boldsymbol{e}_{3}^{r}\right)}^{\psi \boldsymbol{\kappa}_{2}^{r}}+\psi_{, \alpha}\left(\boldsymbol{\gamma}^{r} \cdot \boldsymbol{e}_{3}^{r}\right)]+ \\
& +\mu\left[\psi_{, \alpha}+\psi \boldsymbol{\kappa}_{2}^{r}+\psi_{, \alpha}\left(\boldsymbol{\gamma}^{r} \cdot \boldsymbol{e}_{3}^{r}\right)\right]\left(\boldsymbol{\gamma}^{r} \cdot \boldsymbol{e}_{3}^{r}\right)+ \\
& +\mu\left(\boldsymbol{c}_{\alpha} \cdot \boldsymbol{e}_{3}^{r}\right)+\lambda\left(\boldsymbol{\gamma}^{r} \cdot \psi \boldsymbol{\kappa}^{r} \times \boldsymbol{e}_{3}^{r}+p \psi_{, \beta}^{2}\right) p \psi_{, \alpha}+\lambda(\boldsymbol{I}: \boldsymbol{E}) \psi_{, \alpha}+ \\
& +\mu \psi_{, \beta}\left(\boldsymbol{c}_{\alpha} \cdot \boldsymbol{e}_{\beta}^{r}\right)+\mu p \psi_{, \beta}\left(2 p \psi_{, \beta} \psi_{, \alpha}\right)=\boldsymbol{b}_{\alpha} \\
& \frac{\partial\left(\boldsymbol{\tau}_{\alpha}^{r} \cdot \boldsymbol{e}_{3}^{r}\right)}{\partial p^{\prime}}=\mu\left(p \psi_{, \alpha} \psi\right)+\mu\left[p \psi_{, \alpha} \psi+\left(\boldsymbol{c}_{\alpha} \cdot \boldsymbol{e}_{3}^{r}\right) \psi\right]+\lambda\left[\left(\psi+\psi \gamma^{r} \cdot \boldsymbol{e}_{3}^{r}\right) p \psi_{, \alpha}\right]+o=d_{\alpha}
\end{aligned}
$$

Assim, as submatrizes de $\boldsymbol{D}$ resultam em: 


$$
\begin{aligned}
& \boldsymbol{D}_{\eta \eta}=\frac{\partial \boldsymbol{n}^{r}}{\partial \boldsymbol{\eta}^{r}}=\int_{A} \frac{\partial \boldsymbol{\tau}_{3}^{r}}{\partial \boldsymbol{\gamma}^{r}} \frac{\partial \boldsymbol{\gamma}^{r}}{\partial \boldsymbol{\eta}^{r}} d A=\int_{A} \boldsymbol{C}_{3} d A \\
& \boldsymbol{D}_{\eta \kappa}=\frac{\partial \boldsymbol{n}^{r}}{\partial \boldsymbol{\kappa}^{r}}=\int_{A} \frac{\partial \boldsymbol{\tau}_{3}^{r}}{\partial \boldsymbol{\gamma}^{r}} \frac{\partial \boldsymbol{\gamma}^{r}}{\partial \boldsymbol{\kappa}^{r}} d A=\int_{A}-\boldsymbol{C}_{3}\left(\boldsymbol{A}^{r}+p \psi \boldsymbol{E}_{3}^{r}\right) d A \\
& \boldsymbol{D}_{\eta p}=\frac{\partial \boldsymbol{n}^{r}}{\partial p}=\int_{A} \frac{\partial \boldsymbol{\tau}_{3}^{r}}{\partial p} d A=\int_{A} \boldsymbol{c} d A \\
& \boldsymbol{D}_{\eta p^{\prime}}=\frac{\partial \boldsymbol{n}^{r}}{\partial p^{\prime}}=\int_{A} \frac{\partial \boldsymbol{\tau}_{3}^{r}}{\partial p^{\prime}} d A=\int_{A} \boldsymbol{d} d A \\
& \boldsymbol{D}_{k k}=\frac{\partial \boldsymbol{m}^{r}}{\partial \boldsymbol{\kappa}^{r}}=\int_{A} \frac{\partial}{\partial \boldsymbol{\kappa}^{r}}\left(\boldsymbol{A}^{r}+p \psi \boldsymbol{E}_{3}^{r}\right) \boldsymbol{\tau}_{3}^{r} d A=\int_{A}\left(\boldsymbol{A}^{r}+p \psi \boldsymbol{E}_{3}^{r}\right) \frac{\partial \boldsymbol{\tau}_{3}^{r}}{\partial \boldsymbol{\kappa}^{r}} d A \\
& =\int_{A}-\left(\boldsymbol{A}^{r}+p \psi \boldsymbol{E}_{3}^{r}\right) \boldsymbol{C}_{3}\left(\boldsymbol{A}^{r}+p \psi \boldsymbol{E}_{3}^{r}\right) d A \\
& \boldsymbol{D}_{k p}=\frac{\partial \boldsymbol{m}^{r}}{\partial p}=\int_{A} \frac{\partial}{\partial p}\left(\boldsymbol{A}^{r}+p \psi \boldsymbol{E}_{3}^{r}\right) \boldsymbol{\tau}_{3}^{r} d A= \\
& =\int_{A}^{A}\left[\psi \boldsymbol{E}_{3}^{r} \boldsymbol{\tau}_{3}^{r}+\left(\boldsymbol{A}^{r}+p \psi \boldsymbol{E}_{3}^{r}\right) \boldsymbol{c}\right] d A \\
& \boldsymbol{D}_{k p^{\prime}}=\frac{\partial \boldsymbol{m}^{r}}{\partial p^{\prime}}=\int_{A} \frac{\partial}{\partial p^{\prime}}\left(\boldsymbol{A}^{r}+p \psi \boldsymbol{E}_{3}^{r}\right) \boldsymbol{\tau}_{3}^{r} d A=\int_{A}\left(\boldsymbol{A}^{r}+p \psi \boldsymbol{E}_{3}^{r}\right) \boldsymbol{d} d A \\
& \boldsymbol{D}_{p p}=\frac{\partial Q}{\partial p}=\int \frac{\partial}{\partial p}\left[\left(\boldsymbol{\tau}_{\alpha}^{r} \cdot \boldsymbol{e}_{3}^{r}\right) \psi_{, \alpha}+\boldsymbol{\tau}_{3}^{r} \cdot\left(\boldsymbol{\kappa}^{r} \times \boldsymbol{e}_{3}^{r}\right) \psi\right] d A= \\
& =\int_{A}^{A}\left[\boldsymbol{b}_{\alpha} \psi_{, \alpha}+\left(\boldsymbol{\kappa}^{r} \times \boldsymbol{e}_{3}^{r}\right) \psi \cdot \boldsymbol{c}\right] d A \\
& \boldsymbol{D}_{p p^{\prime}}=\frac{\partial Q}{\partial p^{\prime}}=\int_{A} \frac{\partial}{\partial p^{\prime}}\left[\left(\boldsymbol{\tau}_{\alpha}^{r} \cdot \boldsymbol{e}_{3}^{r}\right) \psi_{, \alpha}+\boldsymbol{\tau}_{3}^{r} \cdot\left(\boldsymbol{\kappa}^{r} \times \boldsymbol{e}_{3}^{r}\right) \psi\right] d A \\
& =\int_{A}^{A}\left[d_{\alpha} \psi_{, \alpha}+\psi\left(\boldsymbol{\kappa}^{r} \times \boldsymbol{e}_{3}^{r}\right) \cdot \boldsymbol{d}_{3}\right] d A \\
& \boldsymbol{D}_{p^{\prime} p^{\prime}}=\frac{\partial B}{\partial p^{\prime}}=\int_{A} \frac{\partial}{\partial p^{\prime}}\left(\boldsymbol{\tau}_{3}^{r} \cdot \boldsymbol{e}_{3}^{r}\right) \psi d A=\int_{A} \psi\left(\boldsymbol{e}_{3}^{r} \cdot \boldsymbol{d}_{3}\right) d A
\end{aligned}
$$

\subsection{Implementação numérica}

A teoria de barras acima apresentada foi codificada no programa de elementos finitos PEFSYS. Uma das desvantagens de se reter todos os termos na equação constitutiva é que se torna necessária a expressão explícita da função empenamento.

Nesse trabalho, em cada segmento retangular da seção transversal (mesa, alma, etc), a função empenamento adotada tem a seguinte expressão: 


$$
\psi(x, y)=\omega_{\text {vlasov }}+\psi_{\text {furia }}(x, y)
$$

onde $\omega_{\text {vlasov }}$ é a área setorial da teoria de Vlasov [23], e $\Psi_{\text {furia }}$ é uma função cúbica que, para domínios retangulares, vale

$$
\psi_{\text {furia }}(x, y)=\psi_{0}-\frac{\left(a^{6}+19 a^{4} b^{2}-19 a^{2} b^{4}-b^{6}\right) x y+\frac{35}{12} a^{2} b^{2}\left(-4 x^{3} y+4 x y^{3}\right)}{a^{6}+14 a^{4} b^{2}+14 a^{2} b^{4}+b^{6}}
$$

onde $a$ é a semi base da seção transversal retangular e $b$ a semi altura. A Figura 3.1 mostra a seção em forma de retângulo

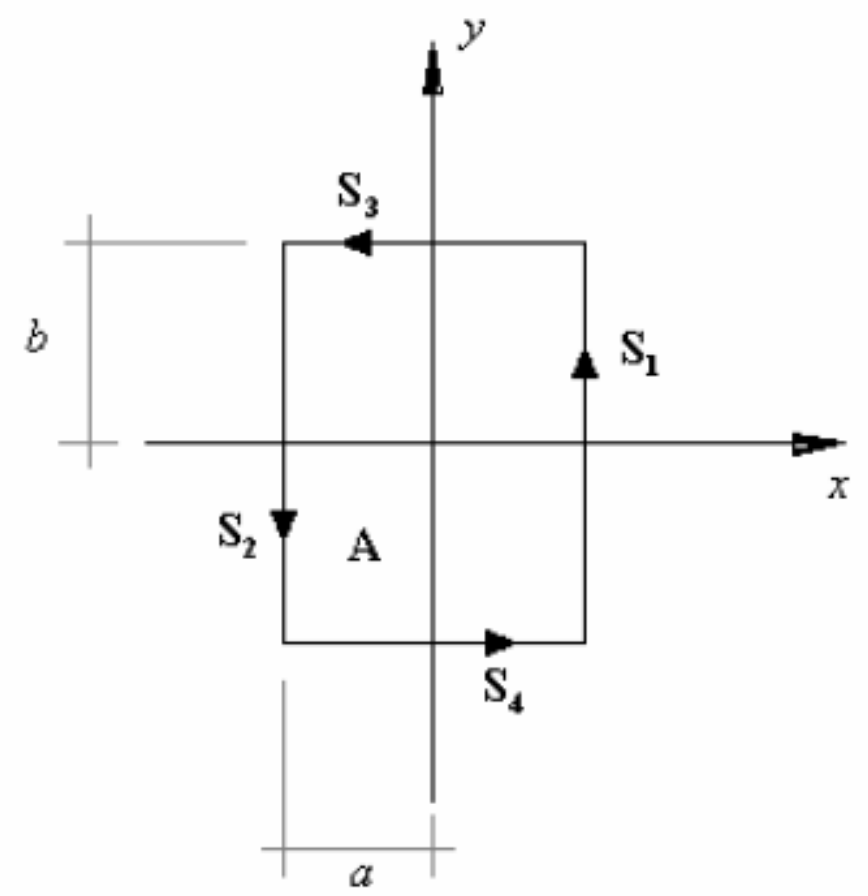

Figura 3.1- Seção em forma de retângulo [26].

O contorno do retângulo é formado pela união de quatro curvas:

$$
S=S_{1} \cup S_{2} \cup S_{3} \cup S_{4}
$$

Essa função (3.30) foi desenvolvida em [26], tendo sido obtida como solução aproximada do seguinte problema 


$$
\begin{array}{ll}
\nabla^{2} \psi=\psi_{, \alpha \alpha}=0 & \text { em } A, \\
\nabla \psi \cdot \boldsymbol{\nu}=\psi_{, \alpha} \nu_{\alpha}=-e_{\alpha \beta}\left(x_{\alpha}-s_{\alpha}\right) v_{\beta} & \text { em } C,
\end{array}
$$

onde $C$ é o contorno da seção, $\nu$ é o versor normal a $C, s_{\alpha}$ são as coordenadas de um ponto conhecido como centro de cisalhamento da seção, e $\psi$ é a função de empenamento relativa ao centro de cisalhamento.

Sabendo-se as coordenadas $s_{\alpha}$ do centro de cisalhamento, o problema (3.32) tem uma solução única a menos de uma constante, a qual pode ser determinada impondo-se

$$
\int_{A} \psi d A=0 .
$$

Por sua vez, o centro de cisalhamento pode ser definido de maneira que

$$
\int_{A} x_{\alpha} \psi d A=0
$$

O que vale dizer que o produto de inércia setorial é nulo para um par de eixos passando pelo centro de cisalhamento [22], e [23].

\subsection{Material hiperelástico de Simo-Ciarlet}

A lei hiperelástica de Simo-Cialert [35] é dada por:

$$
\begin{aligned}
& \boldsymbol{S}=\lambda \frac{1}{2}\left(J^{2}-1\right) \boldsymbol{C}^{-1}+\mu\left(\boldsymbol{I}-\boldsymbol{C}^{-1}\right) \Rightarrow \\
& \boldsymbol{P}=\boldsymbol{F} \boldsymbol{S} \Rightarrow \boldsymbol{P}^{r}=\boldsymbol{F}^{r} \boldsymbol{S}=\boldsymbol{F}^{r}\left[\lambda \frac{1}{2}\left(J^{2}-1\right) \boldsymbol{C}^{-1}+\mu\left(\boldsymbol{I}-\boldsymbol{C}^{-1}\right)\right] .
\end{aligned}
$$

Daqui saem os vetores coluna $\boldsymbol{\tau}_{\alpha}^{r}$ e $\boldsymbol{\tau}_{3}^{r}$, gerando expressões análogas às de (3.17).

Em seguida, procede-se de forma semelhante ao item anterior para obtenção das submatrizes de $\boldsymbol{D}$.

Esta etapa encontra-se em desenvolvimento, será implementada muito em breve no programa PEFSYS, e os resultados obtidos serão incorporados em um artigo para publicação em periódico. 
Deseja-se usar este material pelo fato desse possuir a policonvexidade, não existente no material de St-Venant.

Espera-se com isso comprovar que a inclusão de todos os termos de ordem superior na equação constitutiva de Simo-Ciarlet faz com que esta lei material seja sim adequada para determinação das cargas críticas de flambagem lateral e de flambagem por torção, fato que não ocorria em [2]. 


\section{Exemplos Numéricos}

São apresentados neste capítulo alguns exemplos numéricos clássicos encontrados na literatura, nos quais se utilizou os modelos desenvolvidos neste trabalho para demonstrar a sua eficiência.

Para comprovar a eficiência da equação constitutiva “completa” desenvolvida neste trabalho, foi feita uma comparação com outros dois modelos já existentes no programa PEFSYS, o primeiro com uma equação constitutiva linear com 6 graus de liberdade e o segundo com uma equação constitutiva linear com 7 graus de liberdade incluindo o grau de empenamento. A nomenclatura usada nos gráficos foi de 6GDL Linear para o modelo de 6 graus de liberdade linear, 7GDL Linear para o segundo modelo com 7 graus de liberdade linear e para o modelo desenvolvido neste trabalho utilizou-se a nomenclatura 7GDL completo.

No processamento via elementos finitos, o vão das vigas é dividido igualmente em dez elementos de dois nós (funções de interpolação linear) como mostra a Figura 4.1.

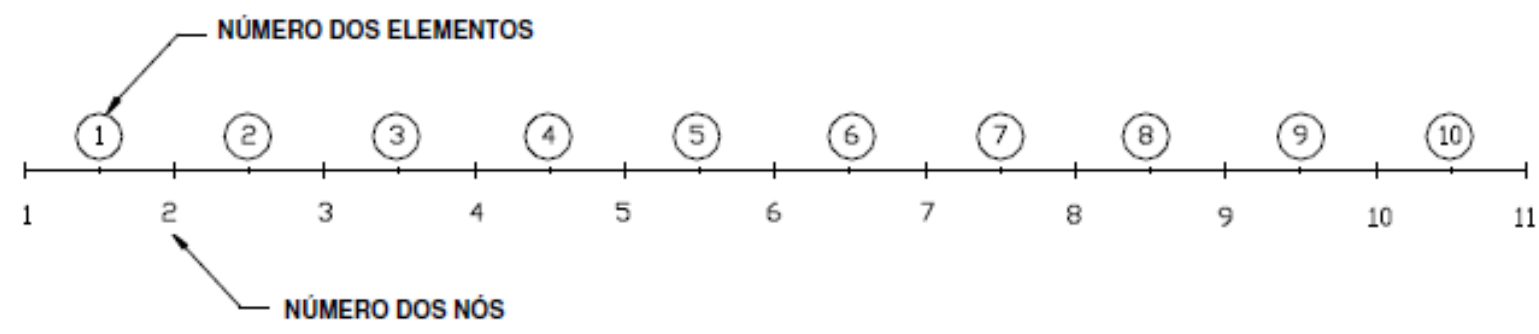

Figura 4.1- Numeração de nós e elementos - PEFSYS.

\subsection{Aferição do modelo}

Com o objetivo de aferir o modelo teórico desenvolvido aqui, foram processados dois perfis de seção I. O primeiro foi o perfil CS 250X52 e o segundo o perfil CVS 150x22.

Neste exemplo analisa-se uma viga metálica de seção transversal I, engastada numa extremidade e livre na outra, com uma força concentrada vertical de $5000 \mathrm{kN}$ aplicada na ponta do balanço. O comprimento total da viga é de $240 \mathrm{~cm}$. Com $E=20000 \mathrm{kN} / \mathrm{cm}^{2}$ e $G=8000 \mathrm{kN} / \mathrm{cm}^{2}$. Foram utilizadas duas seções transversais diferentes, como mostra a Figura 4.2, todas com a carga vertical concentrada aplicada no eixo de simetria da alma. 

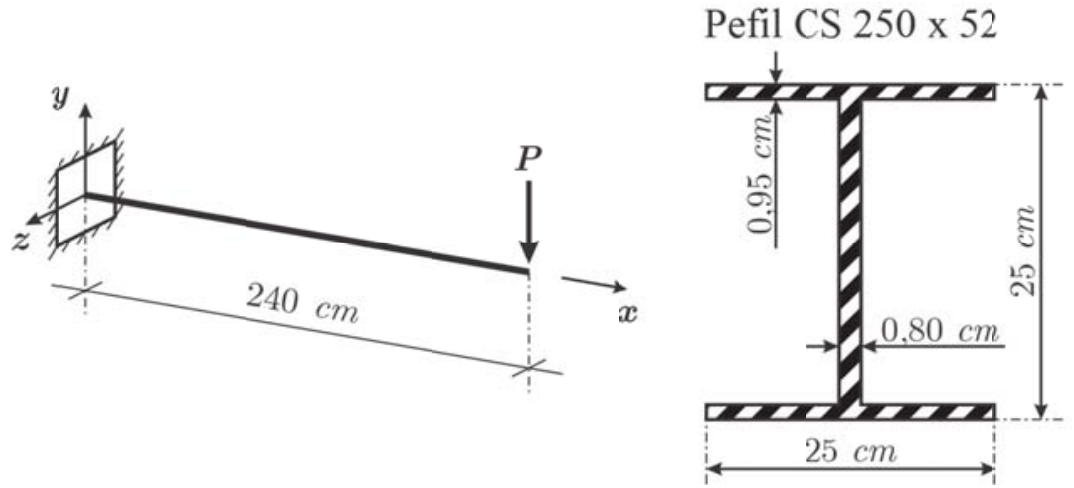

Pefil CVS $150 \times 22$

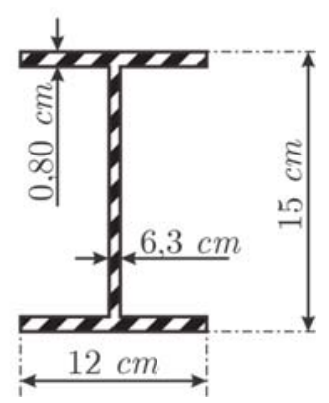

Figura 4.2- Detalhe esquemático do exemplo 4.1.

Em ambos os perfis a viga foi discretizada em seu eixo por 10 elementos lineares de barra, totalizando 11 nós espaçados igualmente. Foi introduzida uma pequena imperfeição geométrica na direção z, para que se pudesse transpor o ponto de bifurcação. Os resultados foram obtidos gerando gráficos de carga versus deslocamento lateral da extremidade, fora do plano (uz). Fazendo a comparação entre os modelos, 6GDL Linear, 7 GDL Linear e o modelo desenvolvido neste trabalho 7GDL completo. As figuras 4.3 e 4.4 ilustram os resultados encontrados.

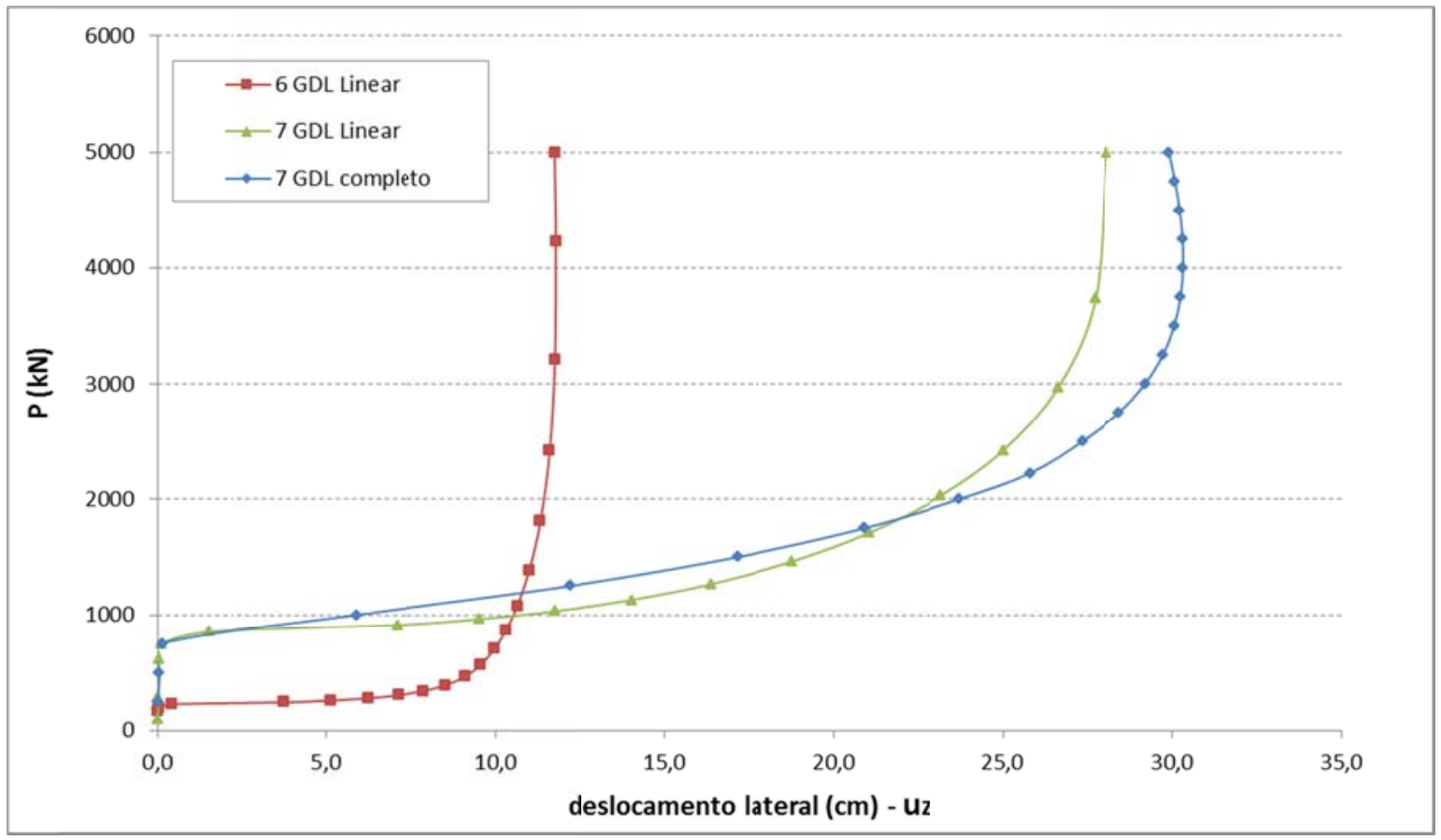

Figura 4.3- Resultados do exemplo 4.1 para o perfil CS 250x52. 


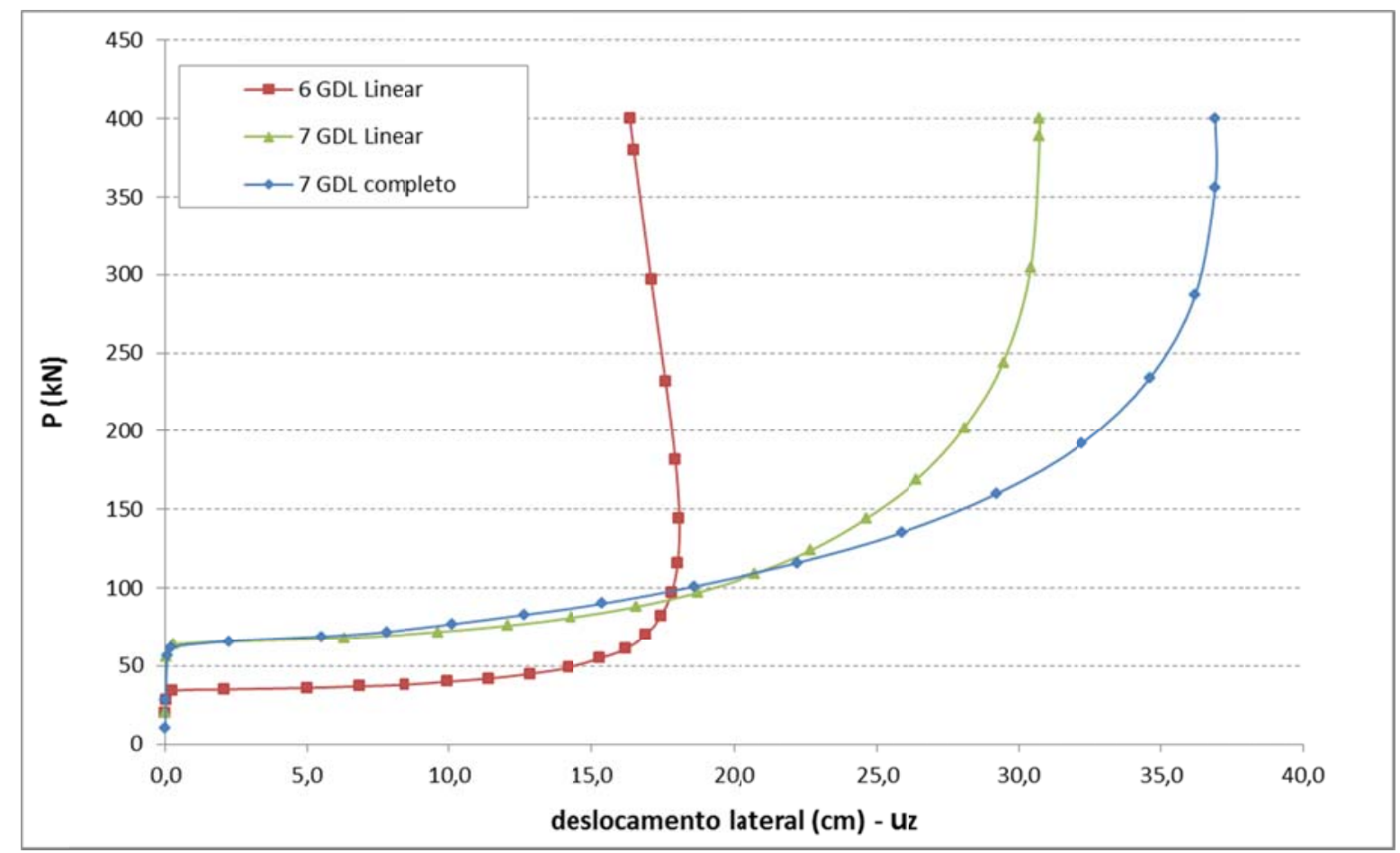

Figura 4.4- Resultados do exemplo 4.1 para o perfil CVS 150x22.

A consideração do empenamento nos deslocamentos nodais prova um aumento na carga crítica de flambagem, para o perfil CS 250x52 a carga crítica para o 6GDL Linear é de 228 kN, enquanto para 7GDL Linear e 7GDL completo são, respectivamente, 854 kN e 865 kN. Para o segundo perfil, CVS 150x22, as cargas para 6GDL Linear, 7GDL Linear e 7 GDL Linar completo são, respectivamente, 35.1 kN, 65.5kN e 66.2 kN .

Percebe-se também que no material com 7GDL completo há um prolongamento maior do ramo pós-crítico em relação ao de 7 GDL Linear, o comportamento com 7 GDL completo apresenta um comportamento mais flexível alcançando maiores deslocamentos laterais, além de uma carga crítica um pouco maior.

\subsection{Flambagem lateral de viga em balanço}

O segundo exemplo deste trabalho foi tirado de [7], apresenta novamente o comportamento de uma viga em balanço sujeito a uma carga vertical concentrada, aplicada no baricentro da extremidade livre. Foram utilizadas duas seções transversais diferentes, como mostra a Figura 4.5, sendo um perfil I (com carga aplicada de $30 \mathrm{kN}$ ) e outro perfil retangular (com carga aplicada de $20 \mathrm{kN}$ ), com o eixo longitudinal passando pelo centro de gravidade. 

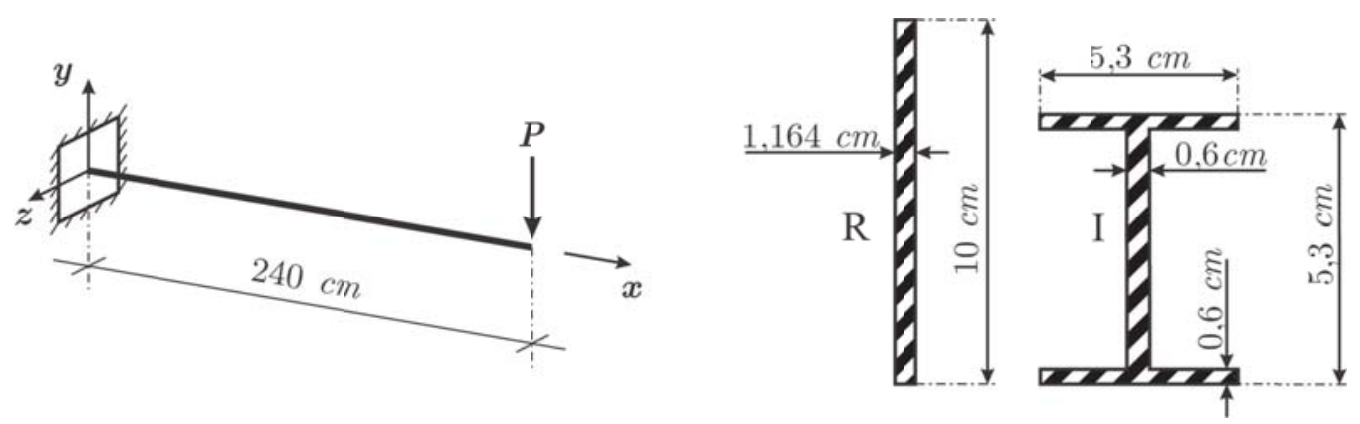

Figura 4.5- Detalhe esquemático do exemplo 4.2.

Utiliza-se $E=20000 \mathrm{kN} / \mathrm{cm}^{2}$ e $G=8000 \mathrm{kN} / \mathrm{cm}^{2}$ e comprimento total de $240 \mathrm{~cm}$. A viga teve a mesma discretização do exemplo anterior. Colocando novamente uma pequena imperfeição geométrica na direção z, para poder transpor o ponto de bifurcação. Os resultados foram obtidos gerando gráficos de carga versus deslocamento lateral da extremidade, ilustrados nas Figuras 4.6 e 4.7.

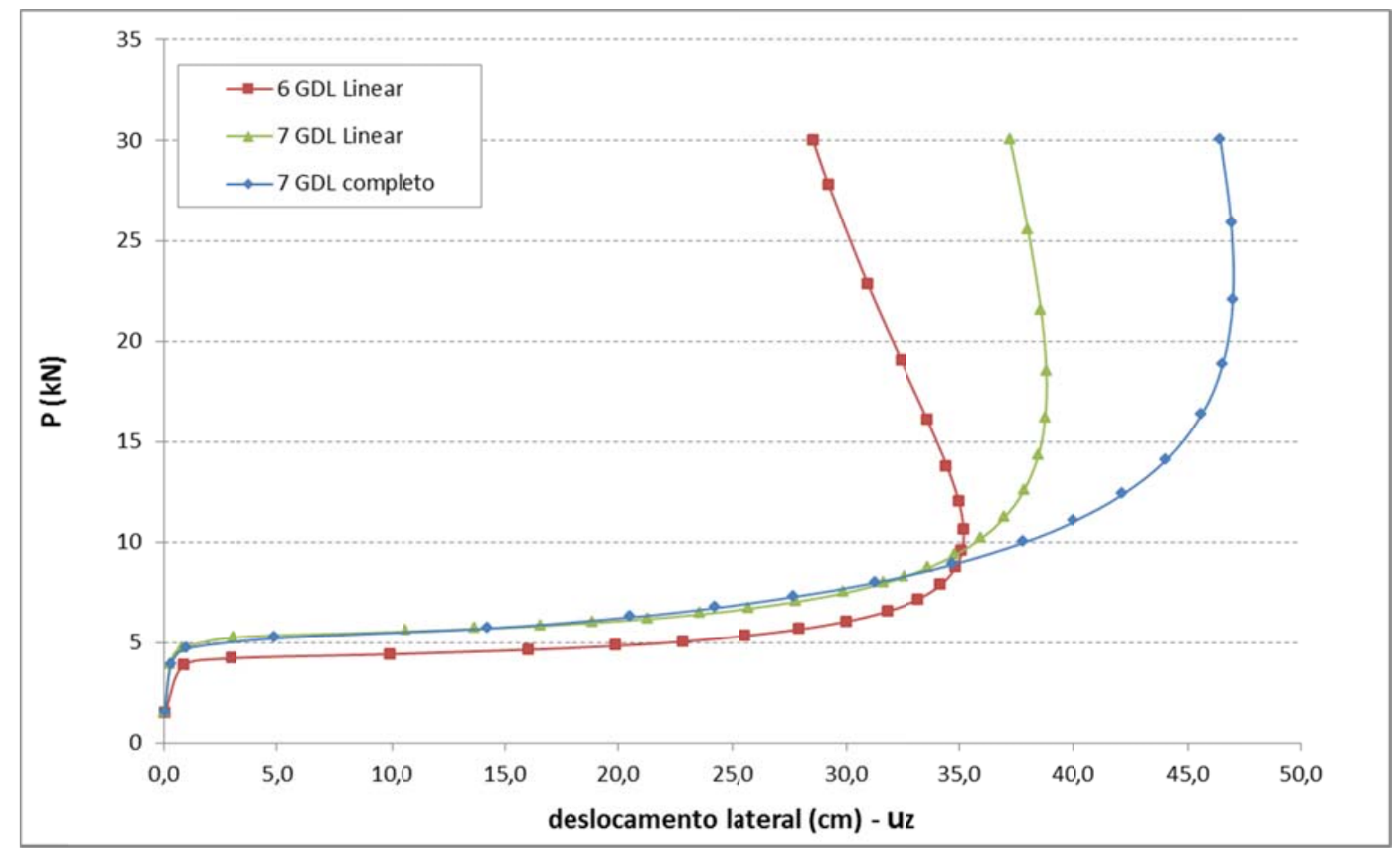

Figura 4.6- Resultados do exemplo 4.2 para o perfil I. 


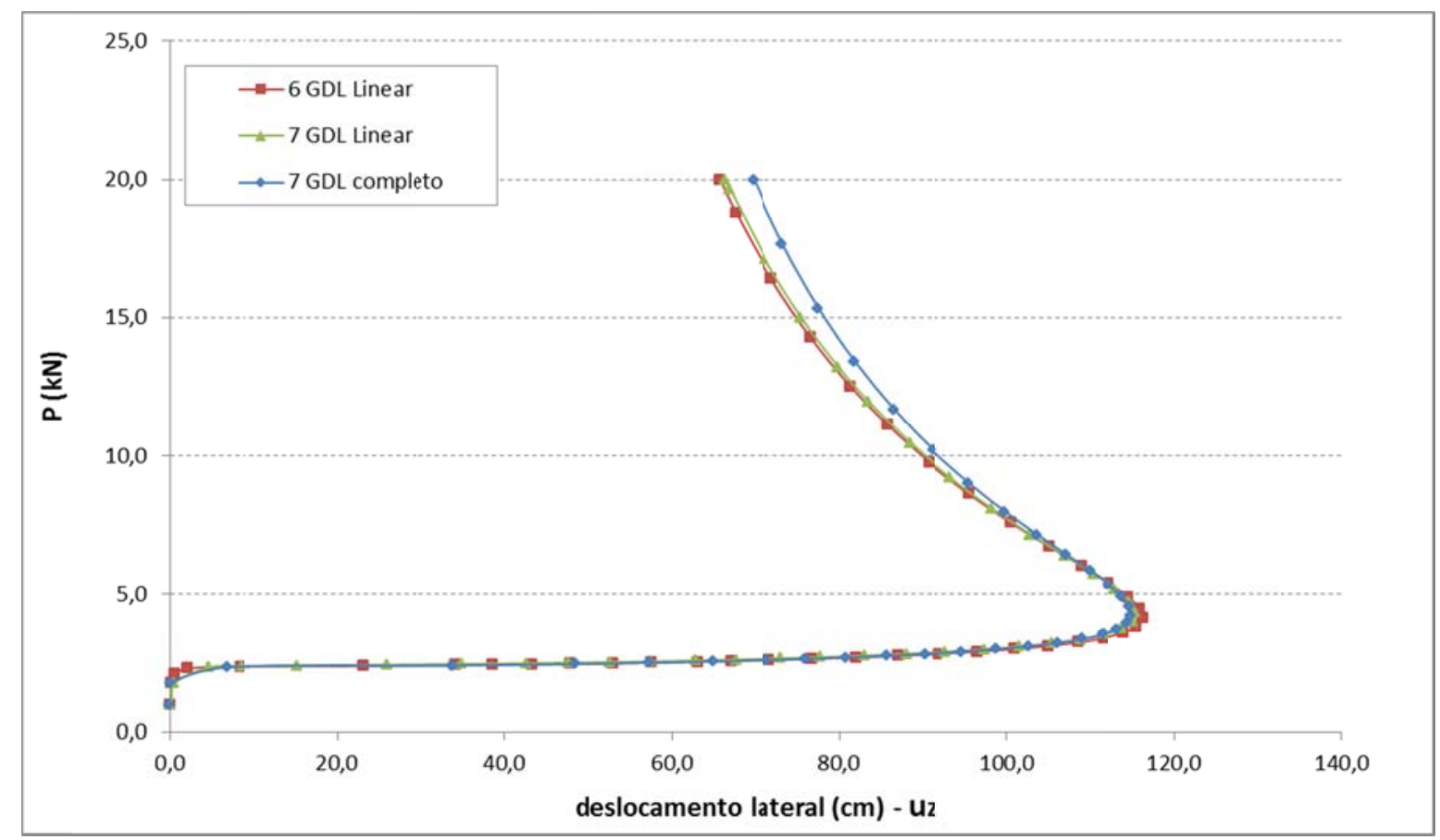

Figura 4.7- Resultados do exemplo 4.2 para o perfil retangular.

A consideração do empenamento provocou um aumento de carga crítica para o perfil I, entretanto para o perfil retangular, as cargas críticas para os três modelos deram iguais devido sua constante ao empenamento ser nula.

O modelo de 7 GDL completo mostrou um comportamento mais prolongado no ramo pós crítico, no caso para o perfil I houve um aumento considerável de deslocamento lateral no modelo em comparação ao de 7 GDL Linear. E para o perfil retangular, mesmo com a constante ao empenamento sendo nula, o modelo com 7GDL completo ainda apresentou um maior deslocamento lateral final no ramo pós-crítico com relação aos dois outros modelos.

\subsection{Flambagem de pilares por compressão com seção I e retangular}

O seguinte problema consiste em um pilar em balanço comprimido por uma força concentrada, aplicado no centro de gravidade da seção extrema. Foram analisados aqui pilares de $240 \mathrm{~cm}$ de comprimento, com as mesmas seções I e retangular do exemplo anterior. Tendo a mesma discretização dos outros exemplos com 11 nós igualmente espaçados. Colocando novamente uma pequena imperfeição geométrica na direção $\mathrm{z}$, para poder transpor o ponto de bifurcação. Adotando-se para as propriedades do material 
$E=20000 \mathrm{kN} / \mathrm{cm}^{2}$ e $G=8000 \mathrm{kN} / \mathrm{cm}^{2}$. Os resultados foram obtidos gerando gráficos de carga versus deslocamento lateral da extremidade, ilustrados nas Figuras 4.8 e 4.9.

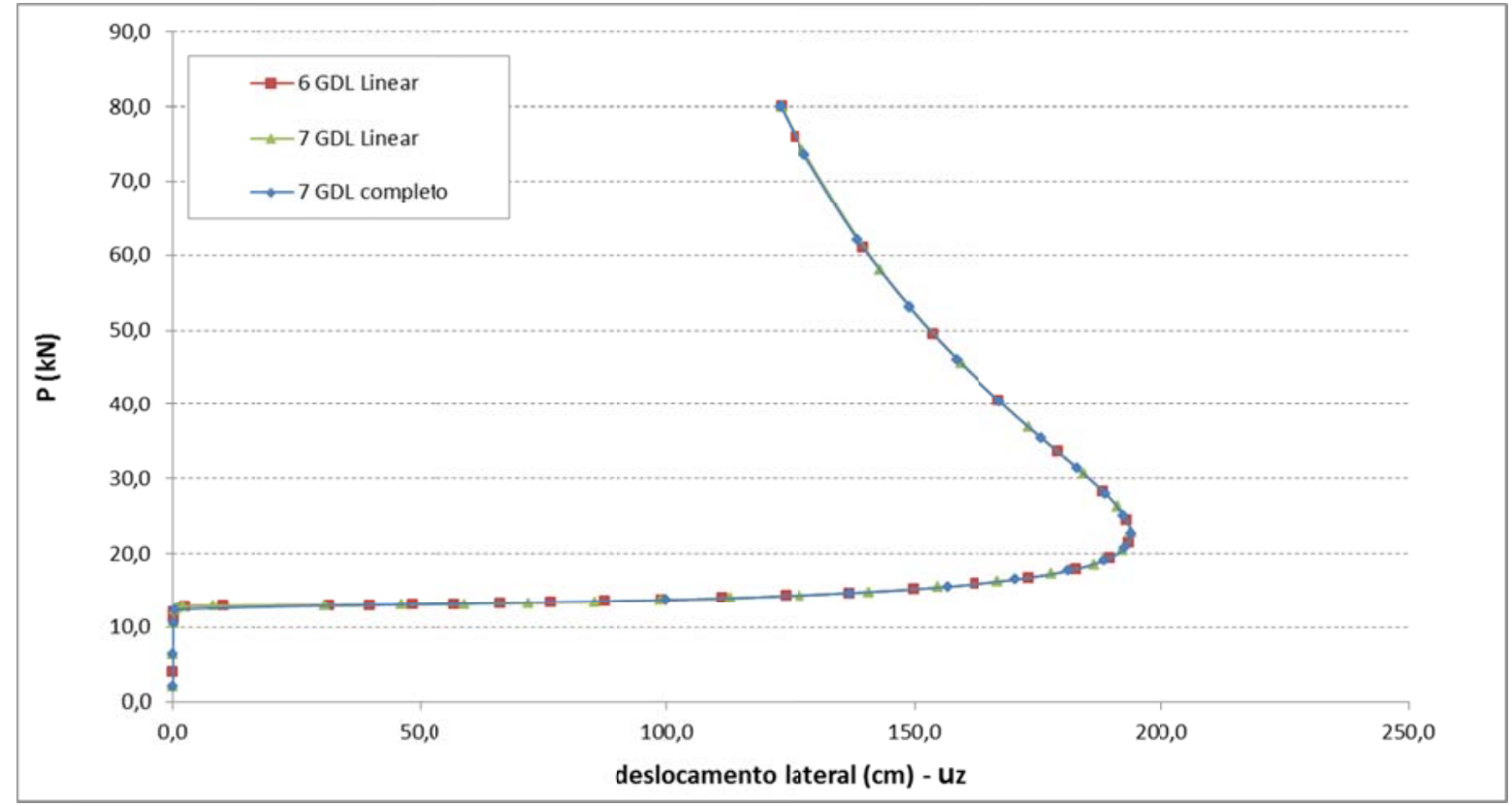

Figura 4.8- Resultados do exemplo 4.3 para o perfil I.

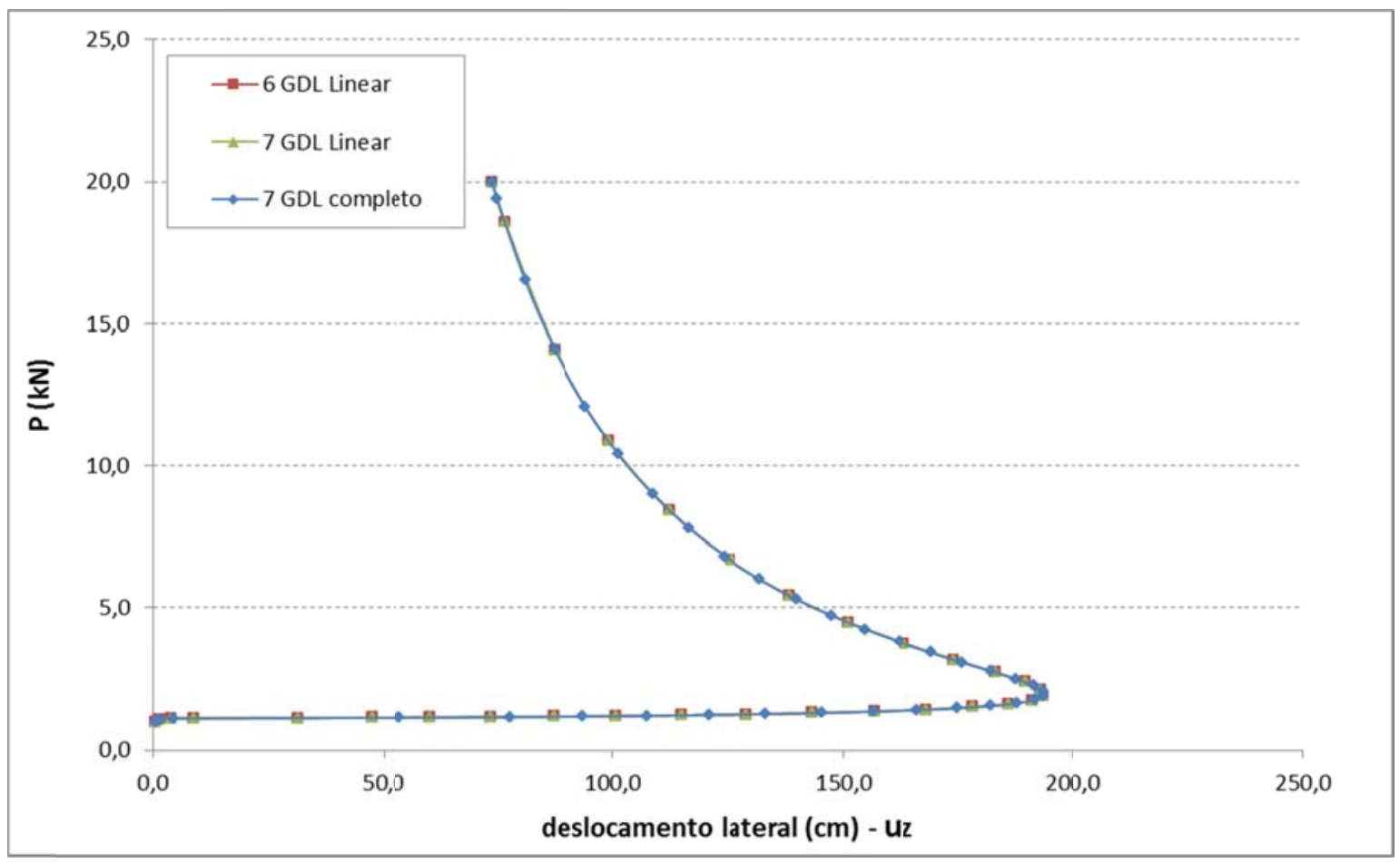

Figura 4.9- Resultados do exemplo 4.3 para o perfil retangular. 
Para os perfis I e retangular submetidos à compressão, aqui estudados, os modelos de 6 GDL Linear, 7GDL Linear e 7GDL completo apresentaram comportamento pós-críticos bastante semelhantes.

\subsection{Flambagem de pilares por compressão com seção cruciforme}

Este problema consiste em um pilar de seção cruciforme comprimido por uma força concentrada como ilustrado na figura 4.10, onde são mostradas as propriedades geométricas e dimensões do problema. Adotando-se para as propriedades também do material $E=20000 \mathrm{kN} / \mathrm{cm}^{2}$ e $G=8000 \mathrm{kN} / \mathrm{cm}^{2}$. Os valores obtidos para as cargas críticas são apresentados na tabela 1.
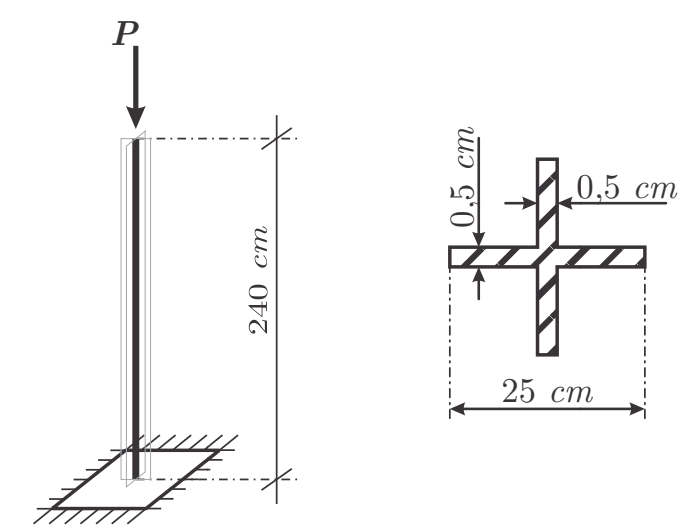

Figura 4.10- Detalhe esquemático do exemplo 4.4.

Tabela 1- Cargas críticas do exemplo 4.4.

\begin{tabular}{|c|c|c|c|c|c|}
\hline Modelo & $\begin{array}{c}6 \mathrm{GDL} \\
\text { Material } \\
\text { linear }\end{array}$ & $\begin{array}{c}7 \mathrm{GDL} \\
\text { Material } \\
\text { linear }\end{array}$ & $\begin{array}{c}7 \mathrm{GDL} \\
\text { material } \\
\text { completo }\end{array}$ & $\frac{\pi^{2} E J}{4 l^{2}}$ & $\frac{G A I_{T}}{I_{x}+I_{y}}$ \\
\hline Pcrit (kN) & 559.3 & 559.3 & 265 & 558 & 316 \\
\hline
\end{tabular}

O modelo com 7 graus de liberdade completo (7 GDL completo) mostrou uma carga crítica menor do que os outros dois modelos, já que este obteve instabilidade por torção neste exemplo com o perfil cruciforme, como mostra a figura 4.11. Partindo para o ramo pós-crítico por instabilidade por flexo-torção, o que não ocorreu nos outros modelos. Nesse exemplo não foi necessário aplicar nenhuma imperfeição geométrica para chegar no ramo pós crítico com o modelo 7GDL completo. 
Pode-se perceber também que a carga crítica do modelo com 7 GDL completo é coerente com a carga crítica teórica da teoria de Vlasov [23], dado pela formula

$$
P_{c r}=\frac{G A I_{T}}{I_{x}+I_{y}},
$$

onde,

$G=$ módulo de elasticidade transversal,

$A$ = área,

$I_{T}=$ inércia a torção,

$I_{x}=$ inércia em torno do eixo $\mathrm{x}$,

$I_{y}=$ inércia em torno do eixo $\mathrm{y}$.

Enquanto os dois outros modelos ficaram com os resultados próximos da carga crítica de Euler.

A Figura 4.11 mostra o gráfico carga crítica versus rotação em torno do eixo x, $\theta_{x}$ (rad). Demostrando que o único modelo que obteve instabilidade por torção foi o 7 GDL completo. Comprovando a eficiência da equação constitutiva “completa”, com todos os termos de ordem superior inclusos. Os modelos 6 GDL Linear e 7GDL Linear não foram necessários para conseguir transpassar para o ramo pós crítico. 


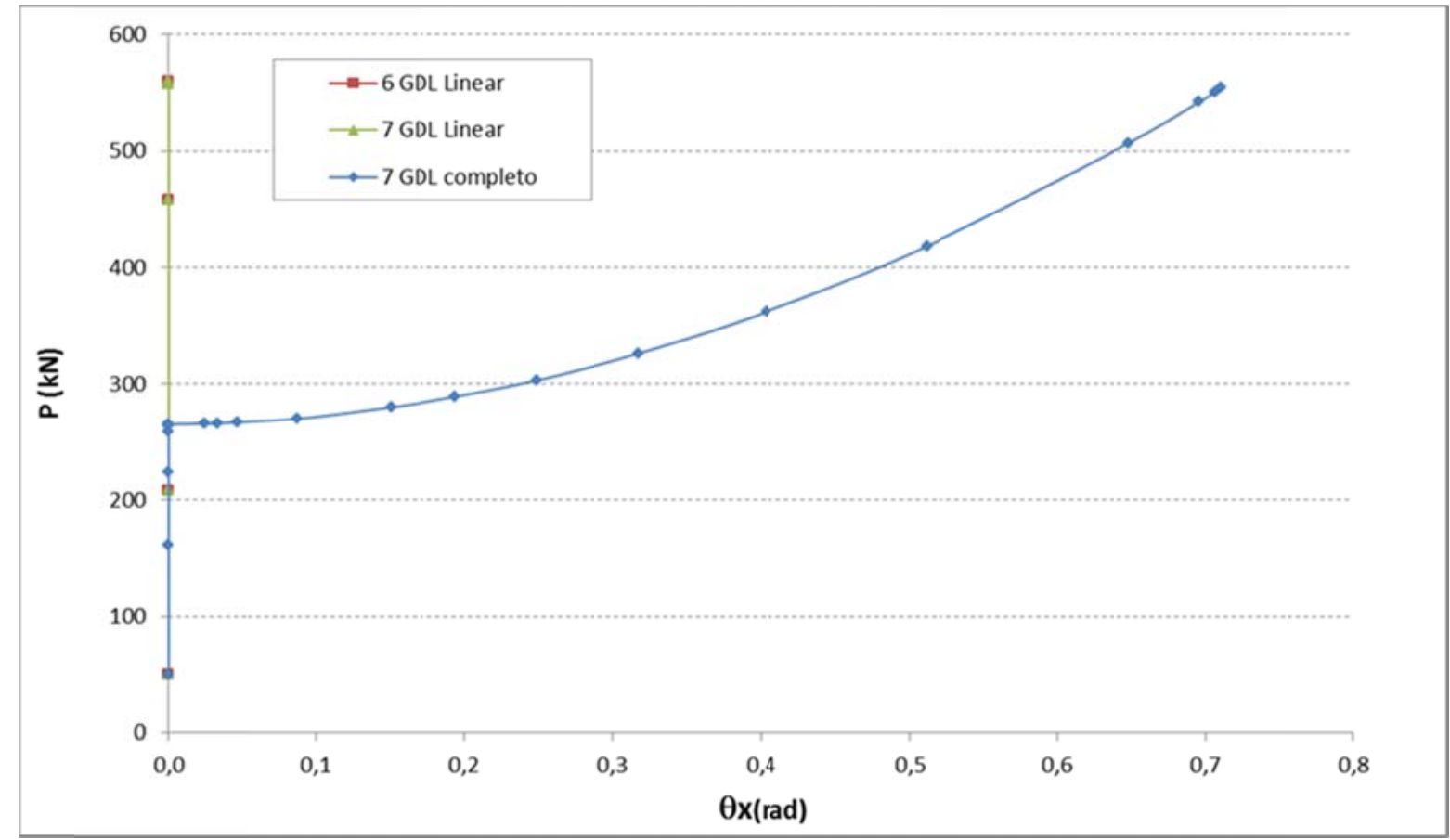

Figura 4.11- Resultados do exemplo 4.4, perfil cruciforme.

Este exemplo foi modelado no programa de elementos finitos Ansys com o objetivo de comparar o modelo aqui desenvolvido. A Figura 4.12 abaixo mostra o exemplo 4.4 modelado no programa Ansys.

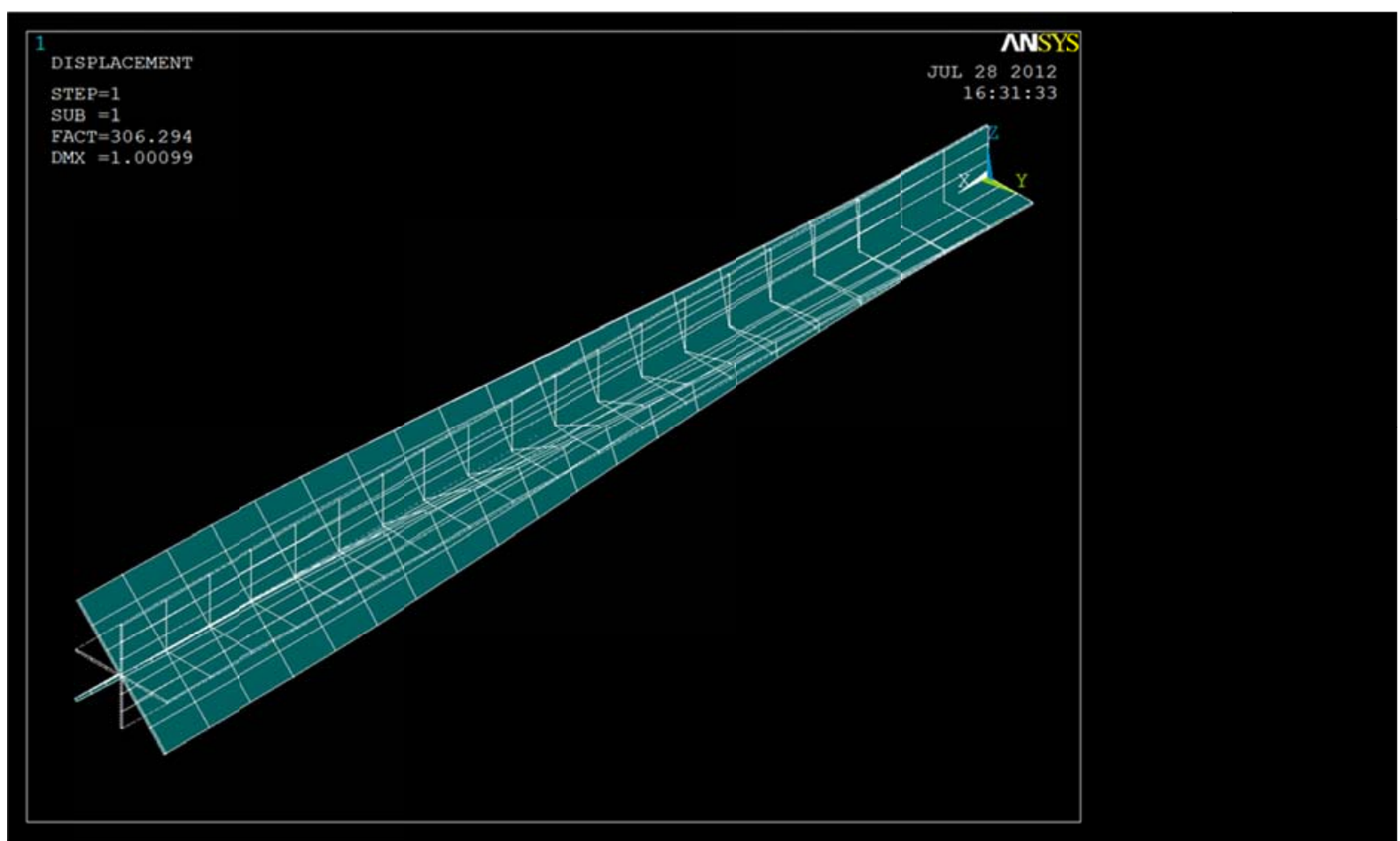

Figura 4.12- Resultados do exemplo 4.4 modelado no Ansys (primeiro modo de instabilidade). 
O primeiro modo de instabilidade no programa Ansys mostrou um resultado para carga crítica próximo do obtido com o modelo 7 GDL completo no programa PEFSYS, comprovando que o modelo constitutivo está certo.

A seguir nas figuras 4.13 e 4.14 é ilustrado o segundo e o terceiro modo de instabilidade do exemplo modelado no programa Ansys.

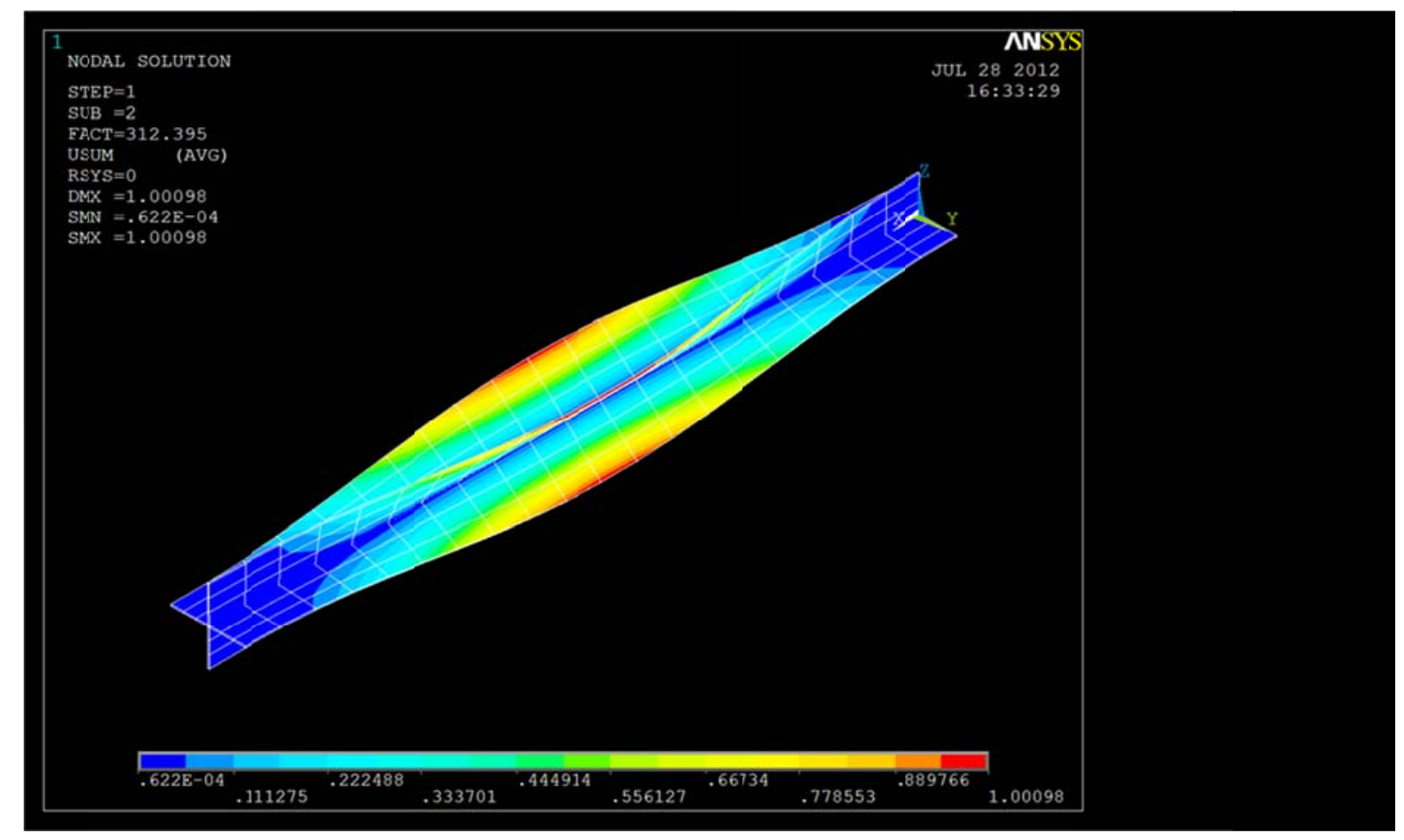

Figura 4.13- Resultados do exemplo 4.4 modelado no Ansys (segundo modo de instabilidade). 


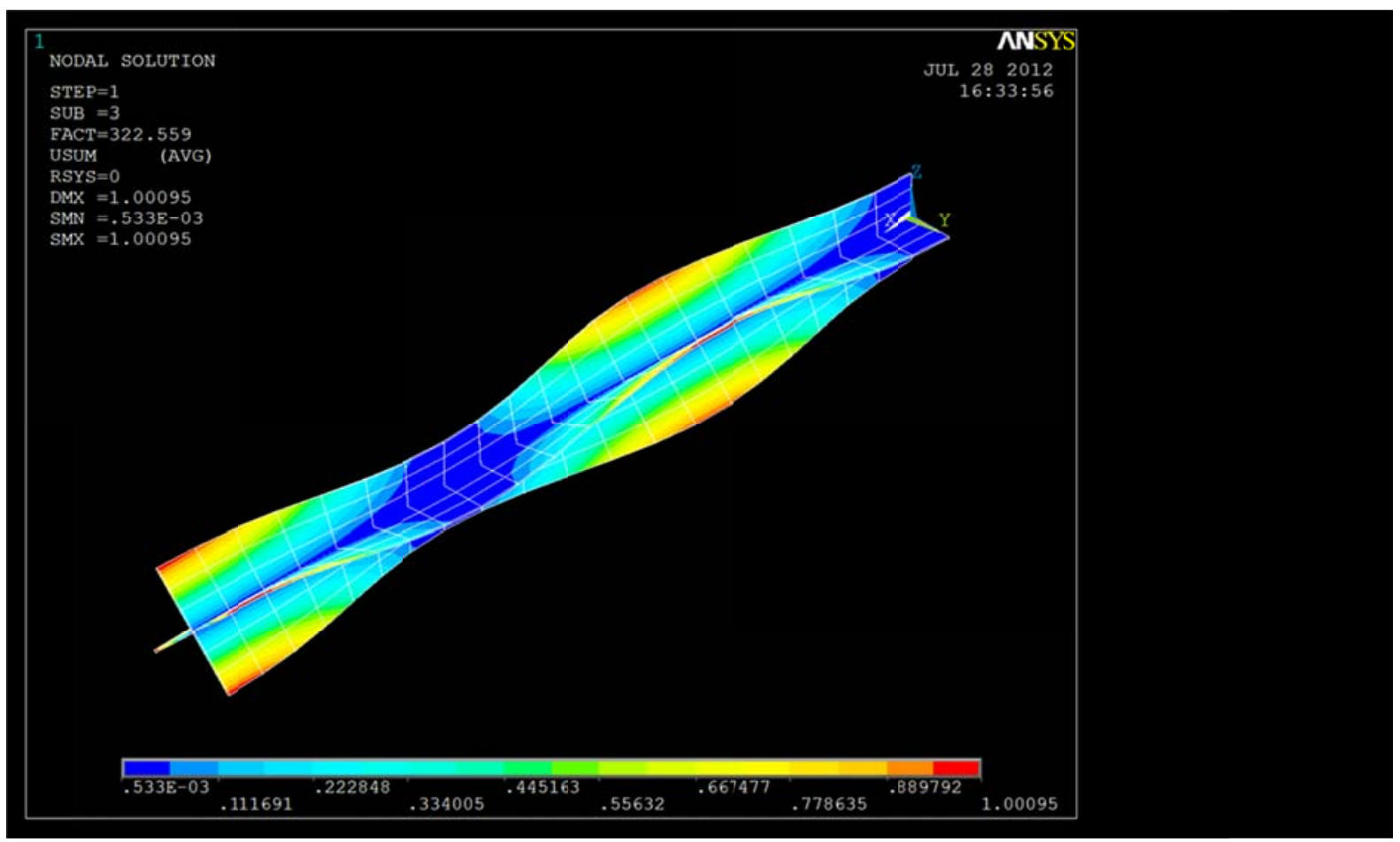

Figura 4.14- Resultados do exemplo 4.4 modelado no Ansys (terceiro modo de instabilidade).

A tabela 2 abaixo mostra o resumo das cargas críticas obtidas no exemplo 4.4.

Tabela 2- Resultados das cargas críticas do exemplo 4.4 e do modelo em Ansys .

\begin{tabular}{|l|c|c|c|c|c|c|}
\hline \multirow{2}{*}{ Modelo } & $\begin{array}{c}6 \mathrm{GDL} \\
\text { Material } \\
\text { linear }\end{array}$ & $\begin{array}{c}7 \mathrm{GDL} \\
\text { Material } \\
\text { linear }\end{array}$ & $\begin{array}{c}7 \mathrm{GDL} \\
\text { material } \\
\text { completo }\end{array}$ & $\begin{array}{c}\text { Modelo } \\
\text { do } \\
\text { Ansys }\end{array}$ & $\frac{\pi^{2} E J}{4 l^{2}}$ & $\frac{G A I_{T}}{I_{\not x}+I_{y}}$ \\
\hline Pcrit (kN) & 559.3 & 559.3 & 265 & 306.3 & 558 & 316 \\
\hline
\end{tabular}




\section{Conclusões}

Conforme apontado em [1] desprezar os termos de segunda ordem nas deformações faz com que a matriz dos coeficientes de rigidez constitutiva $\boldsymbol{D}$ fique incapaz de modelar a instabilidade por torção em barras comprimidas, além de fornecer resultados discrepantes para problemas de flambagem lateral. Conclusões semelhantes também foram alcançadas em [22], porém sem incluir graus de liberdade de empenamento para as seções transversais.

No trabalho de [1], seguiu-se a mesma linha de [24], porém foi proposto generalizar os resultados para o caso da teoria com o empenamento. A única aproximação feita foi a de que os parâmetros $p$ e $p^{\prime}$ relativos ao empenamento somente apresentam contribuição na parcela linear da equação constitutiva. Todos os termos quadráticos e superiores envolvendo estes dois parâmetros foram desprezados, por se julgá-los suficientemente pequeno.

Em [2] seguiu-se a mesma linha de [1], porém foram mantidos os termos de segunda ordem em $p$ e $p^{\prime}$. As cargas de flambagem revelaram ser fortemente afetadas pelos termos de segunda ordem a partir da lei do material. A relação constitutiva elástica linear não permitiu flambagem por torção, enquanto as equações completas até segunda ordem derivadas do material de Saint-Venant e do material de Simo-Ciarlet o fizeram. Contudo, o material de Simo-Ciarlet cotinuou incapaz de modelar problemas de flambagem lateral.

Dando continuidade ao trabalho de [2], este presente trabalho utilizou a mesma formulação de barras incluindo os graus de linerdade de empenamento para as seções transversais e utilizando o material de Saint-Venant; no entanto, aqui todos os termos de ordem superior (segundo e acima) nas deformações foram retidos no nível constitutivo.

A equação constitutiva "completa” desenvolvida neste trabalho provou ser eficaz, obtendo maiores cargas crítica de flambagem com relação ao modelo com equação constitutiva linear com 6 graus de liberdade. Uma das contribuições deste trabalho é na parte do ramo póscrítico, com a equação constitutiva "completa” obteve-se um maior prologamento da curva neste ramo com relação ao modelo de 7 graus de liberdade linear. No caso para o perfil I houve um aumento considerável de deslocamento lateral, para o perfil retangular mesmo a constante ao empenamento sendo nula, o mesmo ainda apresentou um maior deslocamento no ramo pós-crítico para o modelo de 7 graus de liberdade completo em relação aos outros dois modelos comparados. 
Para os perfis submetidos à compressão, aqui estudados, os modelos de 6 GDL Linear, 7GDL Linear e 7GDL completo apresentaram comportamento pós-críticos bastante semelhantes.

A maior contribuição deste trabalho foi demonstrada no exemplo 4.4, onde para o perfil cruciforme o modelo de 7 GDL completo foi o único capaz de chegar na instabilidade por torção, chegando assim ao ramo pós crítico, o que não ocorreu nos outros dois modelos. A carga crítica obtida pelo modelo completo foi perto da carga crítica da teoria de Vlasov, justificando que a equação constitutiva “completa” está correta, enquanto nos modelos de 6 GDL Linear e 7 GDL Linear a carga crítica deu quase idêntica a carga crítica de Euler. Efeito que antes não era possível na equação constitutiva desenvolvida por [2].

Observou-se então que incorporando todos os termos de ordem superior (cúbicos em diante) na equação constitutiva, pode-se obter um comportamento mais flexível alcançando maiores deslocamentos laterais. 


\section{Sugestões para trabalhos futuros}

- Estudar a influência da equação constitutiva “completa” em outros perfis com outros tipos de seções, outros carregamentos e condições de contorno

- Extensão a outros materiais elásticos. 


\section{Referências Bibliográficas}

[1] CAMPELLO EMB. Análise não-linear de perfis metálicos conformados a frio. Dissertação de mestrado, 97p, Escola Politécnica da Universidade de São Paulo. : São Paulo, 2000.

[2] CAMPELLO EMB, PIMENTA PM. Geometrically nonlinear analysis of thin-walled space frames. Proceedings of the Second European Conference on Computational Mechanics (II ECCM) : Cracow, Polônia, 2001.

[3] PFEIL W, PFEIL M. Estruturas de Aço: dimensionamento prático de acordo com a NBR 8800:2008, 8 ed. LTC : Rio de Janeiro, 2009.

[4] BELLEI IH. Edifícios industriais em aço: projeto e cálculo., 5 ed. Pini : São Paulo, 2006.

[5] TIMOSHENKO SP. History of strength of materals. Mc Graw-Hill : New York, 1953.

[6] PRZERMIENIECKI JS. Theory of Matrix Structural Analysis. Dover Publications, Inc : New York, 1985.

[7] YOJO T. Análise não-linear geometricamente exata de pórticos espaciais (com aplicação a torres de transmissão de alta tensão). Tese de doutorado. 129p, Escola Politécnica da Universidade de São Paulo. : São Paulo, 1993.

[8] ZIENKIEWICZ OC. The Finite Element Method, Third Edition ed. McGraw-Hill : New York, 1979.

[9] ARGYRIS JH. Energy theorems and structural analysis. Aircraft Engineering, 26-27. 1955

[10] TURNER MJ,CRW,MHC,ATLJ. Stiffness and deflection analysis of complex structures. Journal of Aeronautical Science:23(9):805-823, 854.

[11] ARGYRIS JH, HILPERT O, MELAJANNAKIS GA, SCHARPF DW. On the geometrical stiffness of a beam im space - a consistent V. W. approach. Computer Methods in Applied Mechanics and Engineering 1979:20

[12] BATHE KJ, BOLOURCHI S. Large displacement analysis of three-dimensional beam structures. Int. J. Numer. Methods. Engrg. 1979:14

[13] ANTMAN SS. Kirchhoff's problem for nonlinearly elastic rods. Quart. J. Appl. Math 1974:32 
[14] REISSNER E. The effect of transverse shear deformations on the bending of elastic plates. $J$. Appl. Mechanics 1945:69-76

[15] SIMO JC. A finite Strain Beam Formulation. The Three-Dimensional Dynamic Problem. Part I. Computer Methods in Applied Mechanics and Engineering, 1985.; 49,:55-70,

[16] SIMO JC, VU-QUOC L. A three- dimensional finite strain rod model. Part II: Computational Aspects. Computational Methods in Applied Mechanics and Engineering, 1986.; 58,:79-116,

[17] SIMO JC, VU-QUOC L. A geometrically exact rod model incorporating shear and torsionwarping deformation. Int. J. Solids and Structures 1991:27

[18] SIMO JC, HUGHES TJR. Formulations of finite elasticity with independent rotations.. Computer Methods In Applied Mechanics and Engineering 1992

[19] PIMENTA PM, YOJO T. Geometrically Exact Analysis of Spatial Frames. Appl. Mech. Reviews 1993

[20] SIMO JC. The (symmetric) Hessian for geometrically nolinear models in solid mechanics: Intrinsic definition and geometric interpretation. Computer Methods in Applied Mechanics and Engineering 1992

[21] GRUTTMANN F, SAUER RAWW. A geometrical nonlinear Eccentric 3D-beam element with arbitrary cross sections. Computer Methods in Applied Mechanics and Engineering 1998

[22] FRUCHTENGARTEN J. Sobre a estabilidade de perfis de seção aberta. Tese de Doutorado, 245p, Escola Politécnica da Universidade de São Paulo. : São Paulo, 1995.

[23] VLASOV VZ. Thin-walled elastic beams. Israel Program for Scientific Translation: Jerusalem, 1961.

[24] PIMENTA PM. Constitutive Second Order Effects on a Geometrically Exact Finite Strain Rod Model. Recent Developments in Solid Mechanics, 79-85, LNCC : Rio de Janeiro, 1996.

[25] LAGO LB, CAMPELLO EMB. Nonlinear analysis of I-section steel beams through the use of non-linear theory of rods. Proceedings of the XXXII Iberian-Latin-American Congress on Computational Methods in Engineering (CILAMCE 2011) : Ouro Preto, 2011.

[26] SILVA HF. Formulação do problema da torção uniforme em barras de seção transversal maciça. Dissertação de mestrado. 203p, Escola Politécnica da Universidade de São Paulo. : São 
Paulo, 2005.

[27] TIMOSHENKO S. Resistência dos Materiais, vol. I. EDITORA TECNOPRINT : 1948.

[28] PIMENTA PM, YOJO T. Geometrically exact analysis of spatial frames with consideration of torsion warping. Proceedings of the XIV Congresso Ibero-Latino Americano de Métodos Computacionais em Engenharia, São Paulo, 1993

[29] MOREIRA MLT. Parametrização das rotações em teorias de barras e cascas. Tese de doutorado, 190p., Escola Politécnica da Universidade de São Paulo. : São Paulo, 2009.

[30] ARGYRIS JH. An excursion into large rotations. Computer Methods in Applied Mechanics and Engineering, 1982.; 32,:85-155,

[31] PIMENTA PM. Rotações Finitas. Boletim Técnico BT/PEF-8714, Departamento de Engenaria de Estruturas e Fundações da Escola Politécnica da Universidade de São Paulo, 1987.

[32] PIMENTA PM. Fundamentos da Teoria das Estruturas. Notas de aula, Departamento de Engenharia de Estruturas e Fundações da Escola Politécnica da Universidade de São Paulo, : 2006.

[33] HUGHES TJR. The Finite Element Method. Englewood Cliffs, New Jersey, 1987.

[34] STARK PA. Introdução aos Métodos Numéricos. Rio de Janeiro, 1979.

[35] SIMO JC, HUGHES TJR. Computational Inelasticity. Springer-Verlag : New York, 1998. 
